\title{
Estrogen receptor inhibition enhances cold-induced adipocyte beiging and
}

\section{glucose sensitivity}

Kfir Lapid ${ }^{1,2, *}$, Ajin Lim², Eric D. Berglund ${ }^{3,4}$ and Jonathan M. Graff ${ }^{1,2,5}$

${ }^{1}$ Division of Endocrinology, Department of Internal Medicine

${ }^{2}$ Department of Developmental Biology

${ }^{3}$ Department of Radiology \& Advanced Imaging Research Center

${ }^{4}$ Department of Pharmacology

${ }^{5}$ Department of Molecular Biology

University of Texas Southwestern Medical Center, Dallas

5323 Harry Hines Boulevard

Dallas, TX 75390-9133 USA

"Correspondence: kfir.lapid@utsouthwestern.edu 


\begin{abstract}
Low estrogen states, exemplified by postmenopausal women, are associated with increased adiposity and metabolic dysfunction. We recently reported a paradox, in which a conditional estrogen receptor-alpha $(\mathrm{ER} \alpha)$ mutant mouse shows a hyper-metabolic phenotype with enhanced brown/beige cell formation ("browning/beiging"). These observations led us to consider that although systemic deficiency of estrogen or ER $\alpha$ in mice results in obesity and glucose intolerance at room-temperature, cold-exposure might induce enhanced browning/beiging and improve glucose metabolism. Remarkably, studying cold-exposure in mouse models of inhibited estrogen signaling - ER $\alpha \mathrm{KO}$ mice, ovariectomy, and treatment with the ER $\alpha$ antagonist Fulvestrant supported this notion. ER $\alpha /$ estrogen deficient mice demonstrated enhanced cold-induced beiging, reduced adiposity and increased glucose sensitivity. Fulvestrant was also effective in diet-induced obesity settings. Mechanistically, ER $\alpha$ inhibition sensitized cell-autonomous beige cell differentiation and stimulation, including $\beta 3$-adrenoreceptor-dependent adipocyte beiging. Taken together, our findings highlight a therapeutic potential for obese/diabetic postmenopausal patients.
\end{abstract}




\section{Introduction}

Obesity and diabetes ("Diabesity") afflict many in western civilizations, and are also a feature of estrogen deficiency ${ }^{1,2}$. Hence, postmenopausal women are prone to show signs of increased adiposity and metabolic dysfunction ${ }^{1,2}$. Accordingly, obesity and metabolic dysfunction develop in pre-clinical estrogen-deficient models, including $\mathrm{ER} \alpha \mathrm{KO}$ and ovariectomized (OVX) female mice $^{3,4}$. Hormone replacement therapy (e.g. estradiol administration) is successful in reversing metabolic dysfunction in rodent models and postmenopausal women ${ }^{1,5}$. Yet, hormone replacement therapy increases the risk of cardiovascular diseases and breast cancer ${ }^{6,7}$. Nonetheless, we recently uncovered an unanticipated function of ER $\alpha$ in systemic metabolism ${ }^{8}$. We found that mutant mice, with low estrogen signaling in the adipose lineage, display a lean phenotype, an improved glucose sensitivity and increased metabolic rates $^{8}$. This paradoxical phenotype appeared secondary to an augmented brown/beige adipocyte differentiation, which appears to occur at the expense of white adipocyte differentiation ${ }^{8}$. Whereas white adipocytes primary function is to store excess energy intake, brown/beige adipocytes convert extra calories into thermal energy, thereby ameliorating diabesity $^{9-13}$. Cold-exposure and sympathetic adrenergic signals are well-established stimuli that activate brown adipose tissue (BAT) and trigger formation of beige adipocyte ${ }^{9-11}$; the latter form within white adipose tissue (WAT). Indeed, cold temperatures are capable of reducing diabesity in rodents and human ${ }^{9-11,14-16}$. Since our aforementioned findings suggest that ER $\alpha$ regulates beiging/browning, we tested whether a browning/beiging stimuli, for example cold-exposure, may improve metabolic outcomes in mouse models of estrogen or estrogen receptor deficiencies. That is, estrogen receptor inhibition renders mice more susceptible to cold-induced browning/beiging effects, which do not normally occur at room temperature. To test our notion, we undertook a series of studies on independent and complementary models: whole-body ER $\alpha \mathrm{KO}$ female mice, 
OVX females, and treatment with the potent estrogen receptor antagonist Fulvestrant ("Faslodex"TM)17. In all models, we observed decreased adiposity and improved metabolic outcomes, which were secondary to enhanced cold-induced beiging and BAT activation. We also probed the effects in a primary beige cell culture, and demonstrated a cell-autonomous beiging following estrogen receptor inhibition. Finally, estrogen receptor inhibition appeared to enhance beiging also via $\beta 3$-adrenoreceptor upregulation and activation.

\section{Methods}

Mice. WT strains include a mixed C57BL/6-129/SV background (for in vitro studies and acute Fulvestrant administration in vivo), ICR(CD1) (for chronic Fulvestrant administration in mice on normal chow) and C57BL/6 (for all other in vivo experiments). ER $\alpha$ KO mice were generously provided by Dr. Deborah J. Clegg ${ }^{18}$. Experiments were performed on 4-6-month-old female mice as indicated. The mice were housed in a temperature-controlled environment using a 12:12 light/dark cycle, and chow and water were provided ad libitum. Bilateral ovariectomy and sham operations were done as instructed in the standard operating procedure. The mice were fed either normal chow (4\% fat, Harlan-Teklad) or high-fat diet (58\% fat with sucrose, Research Diets). For cold experiments, mice were placed in a $6^{\circ} \mathrm{C}$ cold-chamber for 7 days or maintained at $\mathrm{RT}\left(23^{\circ} \mathrm{C}\right)$. Body temperatures were measured using a rectal probe. Fat/lean content/mass were measured using a minispec MQ10 NMR Analyzer (Bruker). Fulvestrant (40 mg/kg/injection, Sigma) or vehicle (DMSO) were dissolved in sunflower oil; acute administration - two intraperitoneal injections initiated 3 days prior to cold-exposure, or chronic administration - four intraperitoneal injections initiated 30 days prior to cold-exposure. CL-316,243 (1 mg/kg/day, Tocris Bioscience) 
was dissolved in $\mathrm{H}_{2} \mathrm{O}$; three daily subcutaneous injections, mice were analyzed a day later. Mice were fasted for $\sim 2 \mathrm{hr}$ at RT prior to euthanization in most experiments, however, prior to glucose uptake assay and glucose/insulin tolerance tests, they were fasted for $\sim 5 \mathrm{hr}$ at RT. For glucose and insulin tolerance tests, $1.25 \mathrm{mg}$ glucose (Sigma) or 0.3-0.75mU Humalog (Lilly)/g mouse weight were injected intraperitoneally; blood glucose levels were measured at the indicated intervals using Contour blood glucose monitoring (Bayer). Glucose uptake assay: jugular vein catheters were surgically implanted in mice a week prior to cold-exposure, using previously described procedures ${ }^{19,20}$. At the end of cold-exposure, mice were injected with a bolus of $13 \mu \mathrm{Ci}$ of $\left[{ }^{14} \mathrm{C}\right]-$ 2-deoxy-D-glucose (2-DG) in the jugular vein catheter. Blood samples were obtained at intervals post-injection, after which mice were euthanized, and tissues were collected. Previously described procedures and calculations were used to determine plasma and tissue radioactivity, and to measure tissue-specific glucose uptake ${ }^{18,19}$. Other measurements were performed in the metabolic phenotyping core: sera insulin, triglycerides and cholesterol and liver triglycerides. All animal procedures were ethically approved by the UT Southwestern Medical Center institutional animal care and use committee.

Cell culture. Stromal vascular (SV) fraction was obtained from subcutaneous adipose depots of two-month old mice as described ${ }^{8,20,21}$. Isolated SV cells were cultured in DMEM supplemented with $10 \%$ FBS, 100 units $/ \mathrm{ml}$ penicillin, $100 \mathrm{mg} / \mathrm{ml}$ streptomycin and $25 \mathrm{ng} / \mathrm{ml}$ Amphotericin B (Sigma). White adipogenesis was induced as described ${ }^{8,21}$. Beige adipogenesis was induced similarly, except for an addition of $5 \mathrm{nM}$ indomethacin (Sigma) and $2 \mathrm{nM}$ T3 (Sigma) ${ }^{20}$. Fulvestrant (25 $\mu \mathrm{M}$, Sigma), Estradiol (1 $\mu \mathrm{M}$, Sigma), CL-316,243 (Tocris Bioscience), SR59230A (1 $\mu$ M, Sigma), Propranolol ( $1 \mu \mathrm{M}$, Cayman Chemical) or vehicles (ethanol, $\mathrm{H}_{2} \mathrm{O}$ or DMSO in equivalent dilutions) were added to confluent cells with each media change. To induce 
thermogenic genes, cells were treated with $10 \mu \mathrm{M}$ Forskolin (Sigma), $1 \mu \mathrm{M}$ Norepinephrine (dissolved in $\mathrm{H}_{2} \mathrm{O}$ with $0.125 \mathrm{mM}$ ascorbic acid, Sigma) or $1 \mu \mathrm{M}$ CL-316,243 - $8 \mathrm{hr}$ for RNA isolation or $24 \mathrm{hr}$ for immunostaining. "Sub-optimal beige media" - IBMX and Dexamethasone (Sigma) were removed. Oil Red O staining, Nile Red staining and immunostaining were done as described $^{8,21}$. Relative changes in cAMP levels were measured using cAMP-Glo ${ }^{\text {TM }}$ Max Assay (Promega) following induction in PBS and IBMX (500 $\mu \mathrm{M}$, Sigma) with or without $10 \mu \mathrm{M}$ Forskolin for $15 \mathrm{~min}$.

Histology, immunohistochemistry and fluorescent immunostaining. Tissues were formalinfixed, paraffin-embedded, sectioned and stained with haematoxylin and eosin (H\&E) as described $^{21}$. Immunohistochemistry and immunofluorescence were done as described ${ }^{20,21}$. Following de-paraffinization, the immunostaining procedure of paraffin-embedded tissue sections was similar to that of frozen tissues and cultured cells. Primary antibodies: rabbit anti-UCP1 (1:200, Abcam), rabbit anti-ER $\alpha$ (1:200, Abcam), rabbit anti-mouse $\beta 3$-adrenergic-receptor (1:200, Abcam), mouse anti-PCNA (1:200, Millipore) and goat-anti-Perilipin (1:200, BD Biosciences). Secondary antibodies: Goat / Donkey anti- rabbit / mouse conjugated with AlexaFluor-488 / Cy3, and Donkey anti-goat conjugated with Cy5 (1:500, Jackson ImmunoResearch). DAPI (1:500, Fluka). Nile-red (1 $\mu \mathrm{g} / \mathrm{ml}$, Sigma). Bright-field and fluorescent images were collected on: Olympus inverted IX70 microscope, Olympus upright BX40 microscope, Leica inverted DMi8 microscope or Leica upright DM6 microscope.

Quantitative teal-time PCR. Total RNA was extracted from adipose tissues or cultured cells as described $^{8,20}$. cDNA synthesis and qPCR analysis of gene expression were done as described ${ }^{8,20}$. qPCR values were normalized to $\beta$-actin. Primer sequences are presented in Supplementary Table 1. 
Statistical analyses and data presentation. Statistical significance was assessed by two-tailed student's t-test, area under curve and linear regression analyses. Error bars indicate S.E.M. Calculations were done and figures were generated using Microsoft Excel 2016, GraphPad Prism 7, ImageJ 1.5, CorelDraw X6 and ChemDraw 15.

\section{Results}

\section{Whole-body ERaKO mice reduce adiposity and increase glucose sensitivity following cold- exposure}

We previously reported that ER $\alpha$ plays alters adipose lineage specification: adipose lineagerestricted (stem cells to mature adipocytes) ER $\alpha$ mutants display increased beige adipocyte formation and BAT activity together with blunted white adipocyte formation ${ }^{8}$. These observations led us to consider that browning/beiging associated with ER $\alpha$ disruption in the adipose lineage might also be present in whole-body $\mathrm{ER} \alpha \mathrm{KO}$ mice under the right conditions. Induction of browning/beiging in ER $\alpha \mathrm{KO}$ females, for example by cold-exposure ${ }^{9}$, is therefore predicted to result in fat loss and improved metabolism, despite their prominent obesity and metabolic dysfunction $^{3,22}$. To test this possibility, we placed 4-5-month-old WT and ER $\alpha$ KO female mice in a cold-chamber for a week or kept them at room-temperature (RT). Cold-exposed ER $\alpha$ KO females significantly reduced body weight, fat content and fat mass, when compared to their counterparts at RT, whereas cold-exposed WT females did not (Fig. 1a-c). Further, the size and mass of subcutaneous and visceral fat depots were significantly decreased in cold-exposed ER $\alpha \mathrm{KO}$ females ( $\sim 3$ fold-change) compared to cold-exposed WT females (Fig. 1d-f). Of note, coldexposure did not appear to affect lean mass and weight of other organs (Fig. S1a-b). Since obesity 
is often associated with manifestation of diabetes, we tested whether the reduction in adiposity of

$\mathrm{ER} \alpha \mathrm{KO}$ females is also associated with improvement of the metabolic profile. Although at RT, we detected hyperglycemia in ER $\alpha \mathrm{KO}$ females, cold-exposure significantly lowered glucose levels, which were similar to the levels measured in cold-exposed WT females (Fig. 1g). We detected similar reductions in serum cholesterol and triglyceride levels in cold-exposed ER $\alpha \mathrm{KO}$ females (Fig. 1h). We additionally performed glucose and insulin tolerance tests at RT or following a week of cold-exposure. Although at RT, ER $\alpha \mathrm{KO}$ females demonstrated glucose intolerance and insulin resistance when compared to the WT counterparts, cold-exposure markedly increased their glucose and insulin sensitivities (Fig. 1i-j). Cold-exposure did not affect the glucose sensitivity of WT females, and only marginally their insulin sensitivity, however, WT females still performed better at these tests, compared to the ERaKO counterparts (Fig. 1i-j). Of note, high insulin levels remained intact following cold-exposure (Fig. S1c). Altogether, we found that upon cold-exposure, female ER $\alpha \mathrm{KO}$ mice exhibited a significant reduction in adiposity and glucose levels together with increased glucose and insulin sensitivities. We had similar observations of reduction in adiposity and metabolic improvement in cold-exposed ER $\alpha$ KO males (not shown). These data suggest that in the absence of ER $\alpha$ mice are more sensitive to the metabolic-positive effects of cold, in contrast to their diabetic phenotype at RT.

\section{Whole-body ERaKO mice exhibit enhanced cold-induced beiging}

According to our hypothesis, the cold-mediated effects on adiposity and glucose metabolism are secondary to an enhanced beiging in $\mathrm{ER} \alpha \mathrm{KO}$ females. At RT, obese ER $\alpha \mathrm{KO}$ females were featured with large adipocytes in the subcutaneous WAT (SWAT) (Fig. 2a). At cold, histological examination of SWAT pointed to emergence of multilocular adipocytes, which were more abundant in ER $\alpha \mathrm{KO}$ females compared to their WT counterparts (Fig. 2a). We did not notice any 
clear differences in the emergence of multilocular adipocytes in the visceral WAT (VWAT) at cold (Fig. 2b). At RT, interscapular classical BAT (or BAT) appeared "whiter" in ER $\alpha$ KO females, however, cold-exposure restored its appearance to a normal morphology (Fig. 2c). UCP1 expression is a hallmark of brown and beige multilocular adipocytes ${ }^{9,13}$. UCP1 immunohistochemistry of SWAT indicated an increased abundance of $\mathrm{UCP}^{+}$beige cells in coldexposed ER $\alpha \mathrm{KO}$ females as compared to cold-exposed WT females (Fig. 2d). A molecular evidence supported the suggested increased beiging: as compared to cold-exposed WT females, cold-exposed ER $\alpha \mathrm{KO}$ females presented elevated mRNA levels of brown/beige cell markers in SWAT, including UCP1 (Fig. 2e). In contrast to SWAT, VWAT did not present an enhanced coldinduced beiging by gene expression (Fig. S2a). Nonetheless, we additionally detected an elevation of gene expression in BAT of ER $\alpha \mathrm{KO}$ females (Fig. 2f). Even though we did not detect UCP1 ${ }^{+}$ cells at RT (Fig. 2d), ER $\alpha$ KO females exhibited increased basal mRNA levels of UCP1 in SWAT (Fig. S2b, applies to other genes too - not shown), but neither in BAT nor in VWAT (not shown). Although increased BAT activity is often associated with a rise in body temperature ${ }^{13}$, coldexposed ER $\alpha \mathrm{KO}$ females reduced their body temperature in a similar manner as WT females (Fig. S2c). We had similar observations of enhanced cold-induced beiging in ER $\alpha \mathrm{KO}$ males (not shown). Our data therefore suggest that reduced adiposity and improved glucose metabolism in $\mathrm{ER} \alpha \mathrm{KO}$ mice at cold are secondary to enhanced cold-induced browning/beiging.

\section{Ovariectomized females reduce adiposity and increase glucose sensitivity following cold-}

\section{exposure}

In addition to a mouse model, lacking the receptor - ER $\alpha$, a model lacking the ligand - estrogen, might serve as a complementary model to test the notion of enhanced cold-induced beiging. A common pre-clinical model in rodents for post-menopause is a surgical removal of the ovaries, 
consequently resulting in lower levels of circulating estradiol ${ }^{23}$. OVX female rodents gradually develop obesity and glucose intolerance, in consistence with metabolic manifestations of diabetic postmenopausal women ${ }^{2,4,5,24}$. Female mice were sham or OVX operated at 2-month of age. Three months later, allowing the OVX females to develop a diabetic phenotype, we placed sham and OVX mice in a cold-chamber for a week or kept them at RT. As expected, at RT, body weight, fat content and mass were higher in OVX females as compared to sham females (Fig. 3a-c). Following cold-exposure, OVX females significantly reduced body weight, fat content and fat mass, when compared to their counterparts at RT, while sham females only marginally (Fig. 3a-c). Further, the size and mass of subcutaneous and visceral fat depots were significantly decreased in cold-exposed OVX females ( 4.5 fold-change) compared to cold-exposed sham females (Fig. 3d-f). Interestingly, the liver, which is fatty in OVX females ${ }^{25}$, reduced its weight following coldexposure (Fig. 3g). Of note, cold-exposure did not appear to affect lean mass and other organs (Fig. S3a-b). Next, we evaluated whether the metabolic state of the OVX females could be improved in a similar manner to ER $\alpha \mathrm{KO}$ females (Fig. 1). While at RT, we detected hyperglycemia in OVX females, cold-exposure significantly lowered glucose levels, which were similar to the levels measured in cold-exposed sham females (Fig. 3h). We detected similar reductions in serum cholesterol and triglyceride levels in cold-exposed OVX females (Fig. 3i). We additionally performed glucose and insulin tolerance tests at RT or following a week of cold-exposure. While at RT, OVX females demonstrated glucose intolerance when compared to the sham counterparts, cold-exposure markedly increased their glucose sensitivity (Fig. 3j). Unlike the positive effect of cold-exposure on the insulin resistance of ER $\alpha \mathrm{KO}$ females (Fig. 1j), cold-exposure did not affect the insulin resistance of OVX females (Fig. S3c). Cold-exposure also did not affect the insulin sensitivity of sham females, and only marginally their glucose sensitivity, however, sham females 
still performed better at these tests, compared to the OVX counterparts (Fig. $3 \mathrm{j}$ and S3c). Of note, high insulin levels remained intact following cold-exposure (Fig. S3d). Altogether, we found that upon cold-exposure, OVX females exhibited a significant reduction in adiposity and glucose levels together with increased glucose sensitivity, suggesting that lower circulating estrogen sensitizes these mice to the metabolic-positive effects of cold.

\section{Ovariectomized females exhibit enhanced cold-induced beiging}

In line with findings in ER $\alpha \mathrm{KO}$ females (Fig. 2), do we observe also enhanced cold-induced beiging in OVX females? At RT, obese OVX females were featured with large adipocytes in the SWAT and VWAT (Fig. 4a-b). At cold, histological examination of SWAT and VWAT pointed to emergence of multilocular adipocytes, which were more abundant in OVX females compared to their sham counterparts (Fig. 4a-b). At RT, BAT appeared "whiter" in OVX females, however, cold-exposure restored its appearance to a normal morphology (Fig. 4c). UCP1 immunohistochemistry of SWAT and VWAT indicated an increased abundance of $\mathrm{UCP} 1^{+}$beige cells in cold-exposed OVX females as compared to cold-exposed sham females (Fig. 4d and S4a). In line with enhanced cold-induced beiging in ERaKO females (Fig. 4e), cold-exposed OVX females presented elevated mRNA levels of brown/beige cell markers in SWAT as compared to cold-exposed sham females (Fig. 4e). We additionally detected an elevation of gene expression in VWAT and BAT of cold-exposed OVX females (Fig. S4b and 4f). As opposed to OVX females, enhanced cold-induced VWAT beiging was not evident in ER $\alpha$ KO females (Fig. 2b and S2a). Another difference between the two models is that OVX females showed similar basal mRNA levels of UCP1 in SWAT at RT (Fig. S4c). Despite increased browning/beiging at cold, OVX females reduced their body temperature in a similar manner as sham females (Fig. S4d). Aligned with reduction in liver weight (Fig. 3g), cold-exposure resulted in correction of estrogen 
deficiency-associated hepatosteatosis (Fig. 4g and S4e). Our data therefore suggest that reduced adiposity and improved glucose metabolism in OVX mice at cold are secondary to enhanced coldinduced browning/beiging.

\section{Fulvestrant enhances beiging in vitro and in vivo}

To test whether estrogen receptor inhibition enhances beiging in a cell-autonomous manner, we utilized a primary beige adipocyte culture. We isolated and cultured SWAT-derived stromal vascular (SV) cells, which are known to contain adipose progenitors ${ }^{21}$. Once the cells reached confluency, we exposed them to beige adipogenic media for a week and then activated them with Forskolin $^{20}$. Since ER $\alpha$-deficient progenitors have compromised adipogenesis ${ }^{8}$, we treated primary cells with the potent estrogen receptor antagonist Fulvestrant ${ }^{17,26}$. Unlike other selective estrogen receptor modulators (SERM), Fulvestrant is capable of ER $\alpha$ downregulation ${ }^{27,28}$, which we confirmed in vitro (Fig. 5a). Treatment with Fulvestrant during differentiation increased the number of $\mathrm{UCP}^{+}$beige cells in culture as compared to controls (Fig. 5b). Gene expression analysis of beige cell markers supported the notion of a cell-autonomous beiging (Fig. 5c). Notably, Fulvestrant did not affect adipogenesis in general (Fig. S5a), and was insufficient to trigger denovo adipogenesis in non-adipogenic media (not shown). Fulvestrant was also incapable of converting white adipocytes to beige adipocytes (Fig. S5b), implying it enhances adipocyte beiging rather than inducing interconversion. Conversely, the ER $\alpha$ ligand, estradiol, has been reported to suppress in vitro white adipogenesis, and our results show that estradiol suppressed in vitro beiging well (Fig. 5b and S5c). Next, we tested whether estrogen receptor inhibition by Fulvestrant can also enhance cold-induced beiging in vivo. We acutely treated 4-month-old females with Fulvestrant or vehicle, and then placed them in a cold-chamber for a week or kept them at RT (Fig. 5d). Following cold-exposure, vehicle-treated and Fulvestrant-treated females 
did not show reduction in body weight (Fig. S5d). Nevertheless, reduction in adiposity was more significant in Fulvestrant-treated females as compared to vehicle-treated females (Fig. 5e-f and S5e). Acute Fulvestrant treatment did not appear to affect other organs (not shown). In accordance with cold effects on ERaKO females (Fig. 2a-b), acute Fulvestrant treatment augmented the emergence of multilocular adipocytes in SWAT at cold (Fig. 5g), but not in VWAT (Fig. S5f). At RT, acute Fulvestrant treatment had no effect on the histological morphology of WAT or BAT (Fig. 5f, S5f and not shown). UCP1 immunohistochemistry of SWAT at cold indicated an increased abundance of $\mathrm{UCP}^{+}{ }^{+}$beige cells in Fulvestrant-treated females as compared to vehicletreated females (Fig. 5h). Furthermore, at cold, Fulvestrant-treated females presented slightly elevated mRNA levels of brown/beige cell markers in SWAT and BAT as compared to vehicletreated females (Fig. 5i-j). We had similar observations of enhanced cold-induced beiging in Fulvestrant-treated males (not shown). As a control experiment, acute administration of Fulvestrant in ER $\alpha \mathrm{KO}$ mice did not result in any additive effect on cold-induced beiging (Fig. 5k and not shown). This supports the hypothesis that the effect of Fulvestrant on beiging is ER $\alpha$ dependent. Taken together, ER $\alpha$ inhibition via Fulvestrant treatments mimics the effects of ER $\alpha$ absence in the enhancement of beiging in vitro and in vivo.

\section{Chronic Fulvestrant treatment reduces adiposity and enhances cold-induced beiging}

Acute Fulvestrant treatment moderately enhanced cold-induced beiging/browning in female mice.

To test the beiging potential of a chronic Fulvestrant treatment, we used an outbred mouse strain of ICR(CD1) that is known to be heavier than the inbred C57Bl/6 and 129/SV strains used so far in this study ${ }^{29}$. The purpose is to demonstrate a generalized strain-independent beiging effect. We treated female mice with Fulvestrant or vehicle for a month (Fig. 6a), considering a long pharmacokinetic half-life of the $\operatorname{drug}^{30}$. Fulvestrant treatment was effective in downregulating ER $\alpha$ 
in WAT and BAT (Fig. S6a-c). We monitored the mice during the chronic Fulvestrant treatment there were no notable changes in body weight, fat mass, fat content, lean mass (Fig. S6d-f), body temperature or food intake (not shown). Then, we placed them in a cold-chamber for a week or kept them at RT. Following cold-exposure, Fulvestrant-treated females significantly reduced body weight, fat content and fat mass, when compared to their counterparts at RT, while vehicle-treated females only marginally (Fig. 6b-d and S6d-f). Further, the size and mass of subcutaneous and visceral fat depots were significantly decreased upon cold-exposure in Fulvestrant-treated females ( $\sim 2$ fold-change) in comparison to vehicle-treated controls (Fig. 6e-g). Of note, Fulvestrant did not appear to affect lean mass and other organs (Fig. S6g-h). In contrast to the blood glucose lowering effect of cold-exposure in ERaKO and OVX females (Fig. 1g and 3h), we did not observe any differences in glucose levels (Fig. 6h); possibly because these mice are not hyperglycemic at RT. Although systemic glycemia did not change, WAT depots demonstrated increased glucose uptake capacity (2.3-3.7 fold-change) in Fulvestrant-treated females as compared to vehicle-treated females at cold conditions (Fig. 6i). Other organs showed no difference in glucose uptake capacity, including BAT (Fig. 6i). This might be due to the timing of the experiment or preference of fatty acid uptake by cold-activated brown adipocytes ${ }^{16}$. At cold, histological examination pointed at striking differences between Fulvestrant-treated females and vehicle-treated females - not only SWAT, but also VWAT displayed high abundance of multilocular adipocytes (Fig. 7a-b). At RT, chronic Fulvestrant treatment had no effect on the histological morphology of WAT or BAT (Fig. 7a-b and not shown). UCP1 immunohistochemistry of SWAT and VWAT at cold indicated an increased abundance of $\mathrm{UCP}^{+}$beige cells in Fulvestrant-treated females as compared to vehicletreated females (Fig. 7c-d). Furthermore, at cold, Fulvestrant-treated females presented elevated mRNA levels of brown/beige cell markers in SWAT, VWAT and BAT as compared to vehicle- 
treated females (Fig. 7e-g). At RT, Fulvestrant-treated females exhibited increased basal mRNA levels of UCP1 in SWAT (Fig. S7a, applies to other genes too - not shown). Thus, chronic Fulvestrant pre-treatment enhanced cold-induced beiging to a larger extent than acute Fulvestrant pre-treatment. What's more, we observed a physiological outcome that we did not observe in $\mathrm{ER} \alpha \mathrm{KO}$ and OVX females - while vehicle-treated females reduced their body temperature upon cold-exposure, Fulvestrant-treated females retained a higher body temperature (Fig. 7h). Monitoring the females at the course of cold-exposure showed that they were more resistant to the ambient cold temperatures (Fig. S7b), implying involvement of enhanced thermogenesis. In conclusion, chronic Fulvestrant treatment in female mice followed by cold-exposure leads to reduced adiposity, enhanced cold-induced beiging of WAT, increased glucose uptake in WAT and increased thermogenesis.

\section{Fulvestrant treatment improves the metabolism of diet-induced obese/diabetic mice}

Our results using Fulvestrant propose that this pharmacological approach is potentially therapeutic. Whereas ER $\alpha \mathrm{KO}$ and OVX females are obese and diabetic, the mice we used to test Fulvestrant so far were raised on normal chow (Fig. 5-7). Hence, we utilized a diet-induced diabesity mouse model fed with a high-fat high-sucrose diet (HFD). Aligning with the surgical OVX model, we fed 2-month-old females with HFD for 3 months, and during the last month, we administered Fulvestrant or a vehicle (Fig. 8a). As expected, HFD resulted in weight gain (Fig. S8a), and Fulvestrant administration had no effect on body weight, fat content, fat mass or lean mass when compared to vehicle treatment (Fig. 8b-c and S8a-c). Then, we placed them in a cold-chamber for a week or kept them at RT. Following cold-exposure, Fulvestrant-treated females reduced body weight and fat mass, when compared to vehicle-treated females at RT (Fig. 8b-c). The reduction in adiposity in females on HFD was moderate (Fig. 8c and S8b,d-e), unlike Fulvestrant treatment 
in females on normal chow (Fig. 6a-g). In accordance with observations in cold-exposed OVX females (Fig. 3g), Fulvestrant treatment in females on HFD reduced liver weight following coldexposure (Fig. S8g), and the the treatment did not appear to affect other organs (not shown). Although Fulvestrant showed a moderate effect over adiposity, we observed a glucose-lowering effect of Fulvestrant in females on HFD, already evident at RT (Fig. 8d). In line with previous reports $^{15,16}$, cold-exposure per se reduced the serum levels of glucose, cholesterol, triglycerides and insulin in mice on HFD (Fig. 8d and S8g-h), however, Fulvestrant treatment had no additive effect. We additionally performed glucose and insulin tolerance tests at RT or following a week of cold-exposure. Cold-exposure is known to increase glucose and insulin sensitivity in mice on $\mathrm{HFD}^{15}$, which we supported in evaluating vehicle-treated females (Fig. 8e-f). Nevertheless, Fulvestrant treatment in females on HFD had a significant synergistic effect in alleviating glucose intolerance at cold (Fig. 8e) and insulin resistance at RT (Fig. 8f), despite similar insulin levels (Fig. S8h). Next, we applied histology and gene expression analysis to females on HFD to evaluate browning/beiging. Histological examination and UCP1 immunoreactivity of SWAT at cold pointed to a scattered emergence of $\mathrm{UCP}^{+}$multilocular adipocytes, but they were not more abundant in Fulvestrant-treated mice (Fig. S8i and not shown). VWAT of females on HFD was resistant to cold-induced beiging, regardless of treatment (not shown). Already evident at RT, the “whiter" BAT, which characterized vehicle-treated females on HFD, was restored to a normal morphology in Fulvestrant-treated females on HFD (Fig. 8g). At cold, BAT morphology appeared even denser in Fulvestrant-treated females on HFD (Fig. 8g). On one hand, at cold, Fulvestranttreated females presented only a moderate elevation in mRNA levels of brown/beige cell markers in SWAT as compared to vehicle-treated females (Fig. 8h). On the other hand, the elevation in the expression of these markers was more notable in BAT at cold (Fig. 8i). At RT, Fulvestrant-treated 
females exhibited increased basal mRNA levels of UCP1 in BAT, but not in SWAT (Fig. S8j, applies to other genes too - not shown). The histological and gene expression analyses suggest that HFD partially inhibited the enhancing effect of Fulvestrant on cold-induced WAT beiging, but not on BAT. Similarly to Fulvestrant-treated females on normal chow (Fig. 7h and S7b), Fulvestrant-treated females on HFD retained a higher body temperature (Fig. 8j and S8k). Moreover, in a similar manner to the observations in OVX females (Fig. 4g and S4e), we observed correction of HFD-associated hepatosteatosis in Fulvestrant-treated females following coldexposure (Fig. 8k and S81). In summary, our study demonstrates that estrogen receptor inhibition leads to reduced adiposity and improved glucose metabolism following cold-exposure, which are secondary to enhanced cold-induced beiging/browning.

\section{Estrogen receptor inhibition promotes beiging via $\beta 3$-adrenoreceptor}

Based on our observations, estrogen receptor inhibition enhanced cold-induced beiging. Adipocyte beiging is associated with multiple signaling pathways, predominantly cAMP-mediated pathways $^{31}$. Indeed, Fulvestrant treatment in vitro elevated basal cAMP levels in differentiated beige cells and Forskolin-induced cAMP levels in undifferentiated cells (Fig. 9a and Fig. S9a), suggesting ER $\alpha$ inhibition "primes" cells to beiging. One of the key stimulants of cAMP-mediated signaling in adipocytes is the sympathetic nervous system, primarily via $\beta$-adrenoreceptors ${ }^{9,13,31}$. We therefore examined whether $\beta$-adrenoreceptors are upregulated in response to in vitro Fulvestrant treatment. We detected a subtype-restricted upregulation of $\beta 3$-adrenoreceptor (AdRß3) in beige cells, whether Fulvestrant is added during differentiation or after they differentiate (Fig. 9b-d). Fulvestrant treatment of non-induced undifferentiated cells also resulted in an upregulation of $\beta$-adrenoreceptors, but not in a subtype-restricted manner (Fig. S9b-d). We should emphasize that the mRNA levels of UCP1 and AdR $\beta 3$ in undifferentiated cells were 
inferior to that of differentiated beige cells (Fig. S9d), and that UCP1 was absent at the protein level in Fulvestrant-treated undifferentiated cells (not shown). PPAR $\gamma 2$ is an essential transcription factor that drives white/beige adipocyte differentiation ${ }^{32-35}$. In differentiated beige cells, Fulvestrant did not affect PPAR 22 expression (Fig. 9b-c), however, Fulvestrant increased its expression in undifferentiated cells (Fig. S9b). Together with no effect on cell proliferation (Fig. S9e) or adipocyte formation (Fig. S5a-b and not shown), these results suggest that Fulvestrant gives a "head-start" in the beiging process. Notably, it is insufficient to trigger beiging by itself, and requires external signals provided by the beige adipogenic media. Could Fulvestrant enhance beiging via $A d R \beta 3$ in a similar manner to cold-induced beiging? Since Fulvestrant pre-treatment upregulated AdRß3 expression (Fig. 9d and S9c), we first pre-treated cells with Fulvestrant or a vehicle, and then stimulated the cells with the specific AdR $\beta 3$ agonist CL-316,243 in the absence of Fulvestrant. Indeed, Fulvestrant pre-treatment increased the number of $\mathrm{UCP}^{+}$beige cells in response to stimulation by CL-316,243, whether it is added during differentiation (Fig. 9e) or prior to induced differentiation (Fig. S9f). In other words, Fulvestrant pre-treatment enhanced CL316,243-induced beiging in vitro. To further support an AdR $\beta 3$-mediated effect, we stimulated cultured beige cells by the natural ligand, Norepinephrine (NE). Not only that Fulvestrant enhanced NE-induced beiging, co-treating beige cells with the specific AdR $\beta 3$ antagonist SR59230A inhibited NE-induced beiging, regardless of treatment (Fig. 9f). Co-treating the cells with a pan-AdR $\beta$ blocker propranolol completely abolished NE-induced beiging (not shown). Next, we tested whether AdR $\beta 3$ stimulation in Fulvestrant-treated females and ER $\alpha$ KO females results in enhanced beiging in a similar manner to cold-exposure. Fulvestrant-treated WT females and ER $\alpha$ KO females showed an upregulated AdR $\beta 3$ expression in SWAT as compared to their control counterparts (Fig. S9g-h). We pre-treated 4-month-old female mice with vehicle or 
Fulvestrant, followed by administration of CL-316,243 injections (Fig. 9g). Following CL316,243 administration, females reduced adiposity, however, reduction in adiposity was more significant in Fulvestrant-pre-treated females as compared to vehicle-pre-treated females (Fig. S9i). We then evaluated SWAT beiging by histological morphology, UCP1 immunostaining and gene expression analysis. Strikingly, Fulvestrant-pre-treated females demonstrated an enhanced beiging response to CL-316,243 administration as compared to vehicle-pre-treated females (Fig. 9h-i and S9j). Despite significant elevation in the expression of brown/beige cell markers in SWAT (Fig. S9j), Fulvestrant pre-treatment did not result in a profound effect on BAT (Fig. S9k). Finally, $\mathrm{ER} \alpha \mathrm{KO}$ females demonstrated an enhanced beiging effect in response to CL-316,243 administration (Fig. 9j-k and not shown). In summary, estrogen receptor inhibition enhances adipocyte beiging in vitro and in vivo via $\operatorname{AdR} \beta 3$ upregulation and activation.

\section{Discussion}

Mouse models of estrogen or ER $\alpha$ deficiencies often develop diabesity, characterized by high fat mass, glucose intolerance, insulin resistance, hyperlipidemia and hepatosteatosis ${ }^{2-4,22,24,25}$. In this study, we show that cold-exposure led to reduced adiposity and improved metabolic profile in ER $\alpha$ KO females, OVX females and Fulvestrant-treated females on HFD (summarized in Fig. 10ab). Supported by our results, cold-exposure by itself is capable of improving glucose metabolism in obese/diabetic rodent models ${ }^{15}$. Yet, our results imply that under circumstances of estrogen receptor inhibition, mice experience a boosted improvement of glucose metabolism in response to cold-exposure. While at "normal" conditions of RT, ER $\alpha \mathrm{KO}$ and OVX female mice were obese/diabetic, at cold, they were more susceptible to its metabolic-beneficial effects. In all these 
models, including regimens of Fulvestrant treatment in female mice on normal-chow, we detected an enhanced cold-induced beiging, predominantly in SWAT, according to histological morphology and gene-expression profiles. We noticed some differences in the manifestation of browning/beiging between these models. For example, cold-induced VWAT beiging occurred only in OVX females and following chronic Fulvestrant pre-treatment in mice on normal-chow. On the other hand, Fulvestrant pre-treatment in mice on HFD resulted in a mild cold-induced beiging, attaining the effects on BAT. While it is conceivable that the enhanced cold-induced beiging and BAT activity exert the observed beneficial effects on glucose metabolism, we cannot exclude that other tissues with ablated estrogen signaling play a cold-inducible role. Tissuespecific ER $\alpha$ mutants, such as deletions of ER $\alpha$ in mature adipocytes, hepatocytes, skeletal muscle and hypothalamic neurons, have been shown to partially develop characteristics of metabolic dysfunction ${ }^{18,36-38}$. Estrogen-dependent regulation of energy balance by the central nervous system is a key physiological contributor, and may explain in part the obese/diabetic phenotype of wholebody ER $\alpha$ KO and OVX mice ${ }^{38-40}$. Nonetheless, Fulvestrant, which does not appear to cross the blood-brain-barrier ${ }^{26}$, has an advantage over other SERMs in our understating of central vs. periphery roles of ER $\alpha$. In support, cerebroventricular injections of Fulvestrant reduce systemic energy expenditure ${ }^{41}$, whereas intraperitoneal injections do not. What's more, Fulvestrant downregulates ER $\alpha$ expression ${ }^{27,28}$, including in our study, and therefore serves as a reliable model for ER $\alpha$ deficiency. We presented a cell-autonomous beiging response under conditions of ER $\alpha$ deficiency by utilizing an isolated primary cell culture. We previously reported an enhanced beiging in mice with an adipose-lineage-specific deletion of $E R \alpha^{8}$, and herein we further validate this beiging effect in mice with systemic ER $\alpha$-deficiency followed by stimuli of beiging/browning. These stimuli, elicited by cold-exposure or adrenergic signals, are necessary to amplify the 
downstream signaling triggered by $\mathrm{ER} \alpha$ inhibition, which do not generate a beiging phenotype under non-stimulatory conditions (i.e. RT). This unknown downstream signaling is yet to be elucidated, however, our preliminary findings indicated upregulated cAMP levels, which are a driving force in beiging, as well as an increased AdR $\beta 3$ expression. Although AdR $\beta 3$ signaling triggers prominent beiging ${ }^{13,31,42}$, AdR $\beta 3-\mathrm{KO}$ mice and mice treated with AdR $\beta 3$ antagonist display normal cold-induced beiging ${ }^{43,44}$, suggesting $\operatorname{AdR} \beta 3$ is redundant in the specific response to cold-exposure. The findings in this study are not contradictory, since they propose that following ER $\alpha$ inhibition, brown/beige adipocytes are more sensitive to activation - either by cold-exposure or by AdR $\beta 3$ agonist. This increased sensitivity may occur both at the immature and mature cell level (Fig. 10c), although this cellular mechanism should be further validated in vivo. Altogether, our results suggest that pre-clinical mouse models of post-menopause have a propensity to increased beiging and improved metabolism upon cold-exposure. Thus, we conclude that coldexposure has a clinical potential in obese/diabetic postmenopausal patients.

\section{Acknowledgements}

This study was supported by NIH, NIDDK (R01 DK066556 and R01 DK088220) and an AHA postdoctoral fellowship grant (K.L., 16POST27250024). We thank Brianna Findley and Xiaoli Lin from the Radiology \& Advanced Imaging Research Center for their assistance in conducting the glucose uptake assay.

\section{Author contributions}

J.M.G. and K.L. conceived, designed and interpreted the experiments. K.L. performed the experiments. A.L initiated this study. E.D.B oversaw the glucose uptake assay. J.M.G. and K.L. analyzed the experiments, discussed the results, and wrote the manuscript. 


\section{Competing financial interests}

J.M.G. is a cofounder and shareholder of Reata Pharmaceuticals. The other authors declare no competing financial interests.

\section{Figure Legends}

Figure 1. Whole-body ERaKO mice reduce adiposity and increase glucose sensitivity following cold-exposure. Four to five-month-old female ER $\alpha \mathrm{WT}(\mathrm{WT})$ and $\mathrm{ER} \alpha \mathrm{KO}(\mathrm{KO})$ mice were subjected to cold-exposure $\left(6^{\circ} \mathrm{C}\right)$ for 7 days or maintained at RT $\left(23^{\circ} \mathrm{C}\right)$. (a) Body weight, $n \geq 12$. (b) Fat mass by NMR, $n \geq 8$. (c) Fat content by NMR, $n \geq 8$. (d) Representative photographs of inguinal (IGW) and perigonadal (PGW) white adipose depots of WT and KO females. (e) Weight of indicated fat depots in WT and KO females: SWAT - IGW and intrascapular (ISCW); VWAT - PGW, retroperitoneal (RPW) and mesenteric (MWAT); and intrascapular BAT, n $\geq 8$. (f) Relative fat mass loss in SWAT and VWAT compartments of WT and KO females were calculated according to white adipose depot weights in (e): SWAT $=\operatorname{Cold}(2 * \mathrm{IGW}+\mathrm{ISCW})$ $\mathrm{RT}(2 * \mathrm{IGW}+\mathrm{ISCW}) ; \mathrm{VWAT}=\operatorname{Cold}(2 * \mathrm{PGW}+2 * \mathrm{RPW}+\mathrm{MWAT})-\mathrm{RT}(2 * \mathrm{PGW}+2 * \mathrm{RPW}+\mathrm{MWAT})$, $n \geq 8$. (g) Blood glucose levels of WT and KO females, $n \geq 6$. (h) Serum cholesterol and triglyceride levels of WT and KO females, $\mathrm{n} \geq 4$. (i-j) Glucose tolerance (i) and insulin tolerance (j) tests were performed in WT and KO females at RT (a week prior to cold-exposure) and immediately after cold-exposure. Mice were fasted, i.p. injected with $1.25 \mathrm{~g} / \mathrm{kg}$ glucose (i) or $0.3 \mathrm{U} / \mathrm{kg}$ insulin (j), and their glucose levels were monitored, $\mathrm{n} \geq 8$. Insets - areas under curve. Error bars indicate S.E.M. Statistical significance assessed by two-tailed student's t-test, * $\mathrm{p}<0.05, * * \mathrm{p}<0.01$.

Figure S1. Related to Figure 1. Four to five-month-old female ER $\alpha \mathrm{WT}$ (WT) and ER $\alpha \mathrm{KO}(\mathrm{KO})$ mice were subjected to cold-exposure $\left(6^{\circ} \mathrm{C}\right)$ for 7 days or maintained at RT $\left(23^{\circ} \mathrm{C}\right)$. (a) Lean mass 
by NMR, $n \geq 8$. (b) Weight of indicated organs in WT and KO females, $n \geq 7$. (c) Serum insulin levels of WT and KO females, $\mathrm{n} \geq 5$. Error bars indicate S.E.M. Statistical significance assessed by two-tailed student's t-test, $* \mathrm{p}<0.05, * * \mathrm{p}<0.01$

Figure 2. Whole-body ERaKO mice exhibit enhanced cold-induced beiging. Four to fivemonth-old female ER $\alpha \mathrm{WT}(\mathrm{WT})$ and $\mathrm{ER} \alpha \mathrm{KO}(\mathrm{KO})$ mice were subjected to cold-exposure $\left(6^{\circ} \mathrm{C}\right)$ for 7 days or maintained at RT $\left(23^{\circ} \mathrm{C}\right)$. (a-c) Representative H\&E-stained histological sections of SWAT (a), VWAT (b) and BAT (c). (d) UCP1 immunohistochemistry - representative sections of SWAT. (e-f) Relative mRNA levels, quantified by qPCR, of brown/beige adipocyte markers expressed in SWAT (IGW, e) and BAT (f) of WT and KO females at cold, $\mathrm{n} \geq 6$. Scale bars $=100$ $\mu \mathrm{m}$. Error bars indicate S.E.M. Statistical significance assessed by two-tailed student's t-test, * p $<0.05, * * \mathrm{p}<0.01$

Figure S2. Related to Figure 2. Four to five-month-old female and male ER $\alpha$ WT (WT) and $\mathrm{ER} \alpha \mathrm{KO}(\mathrm{KO})$ mice were subjected to cold-exposure $\left(6^{\circ} \mathrm{C}\right)$ for 7 days or maintained at $\mathrm{RT}\left(23^{\circ} \mathrm{C}\right)$. (a) Relative mRNA levels, quantified by qPCR, of brown/beige adipocyte markers expressed in VWAT (PGW) of WT and KO females at cold, $\mathrm{n} \geq 6$. (b) Relative mRNA levels, quantified by qPCR, of UCP1 in SWAT (IGW) of WT and KO females at RT and cold, $\mathrm{n} \geq 5$. (c) Body temperature (rectal probe) of WT and KO females at RT and cold, $\mathrm{n} \geq 9$. Scale bars $=100 \mu \mathrm{m}$. Error bars indicate S.E.M. Statistical significance assessed by two-tailed student's t-test, $* \mathrm{p}<0.05, * *$ $\mathrm{p}<0.01$.

Figure 3. Ovariectomized females reduce adiposity and increase glucose sensitivity following cold-exposure. Two-month-old female mice underwent sham operation or ovariectomy (OVX), 3 months later both groups were either subjected to cold-exposure $\left(6^{\circ} \mathrm{C}\right)$ for 7 days or maintained at RT $\left(23^{\circ} \mathrm{C}\right)$. (a) Body weight, $n \geq 11$. (b) Fat mass by NMR, $n \geq 6$. (c) Fat content by NMR, $\geq 6$. (d) 
Representative photographs of IGW and PGW adipose depots of Sham and OVX females. (e) Weight of indicated fat depots in Sham and OVX females: SWAT - IGW and ISCW; VWAT PGW, RPW and MWAT; and intrascapular BAT, $\mathrm{n} \geq 8$. (f) Relative fat mass loss in SWAT and VWAT compartments of Sham and OVX females were calculated according to white adipose depot weights in (e): SWAT $=$ Cold $(2 * I G W+I S C W)-R T(2 * I G W+I S C W) ;$ VWAT = $\operatorname{Cold}(2 * \mathrm{PGW}+2 * \mathrm{RPW}+\mathrm{MWAT})-\mathrm{RT}(2 * \mathrm{PGW}+2 * \mathrm{RPW}+\mathrm{MWAT}), \mathrm{n} \geq 8$. (g) Liver weights of Sham and OVX females, $n \geq 8$. (h) Blood glucose levels of Sham and OVX females, $n \geq 8$. (i) Serum cholesterol and triglyceride levels of Sham and OVX females, $n \geq 8$. (j) Glucose tolerance tests were performed in Sham and OVX females at RT (a week prior to cold-exposure) and immediately after cold-exposure. Mice were fasted, i.p. injected with $1.25 \mathrm{~g} / \mathrm{kg}$ glucose, and their glucose levels were monitored, $n \geq 9$. Inset - areas under curve. Error bars indicate S.E.M. Statistical significance assessed by two-tailed student's t-test, $* \mathrm{p}<0.05, * * \mathrm{p}<0.01, \mathrm{NS}-$ not significant.

Figure S3. Related to Figure 3. Two-month-old female mice underwent sham operation or ovariectomy (OVX), 3 months later both groups were either subjected to cold-exposure $\left(6^{\circ} \mathrm{C}\right)$ for 7 days or maintained at $\mathrm{RT}\left(23^{\circ} \mathrm{C}\right)$. (a) Lean mass by NMR, $\mathrm{n} \geq 6$. (b) Weight of indicated organs in Sham and OVX females, $\mathrm{n} \geq 8$. (c) Insulin tolerance tests were performed in 6-month-old Sham and OVX females at RT (a week prior to cold-exposure) and immediately after cold-exposure. Mice were fasted, i.p. injected with $0.75 \mathrm{U} / \mathrm{kg}$ insulin, and their glucose levels were monitored, $\mathrm{n} \geq 10$. Inset - areas under curve. (d) Serum insulin levels of Sham and OVX females, $\mathrm{n} \geq 5$. Error bars indicate S.E.M. Statistical significance assessed by two-tailed student's t-test, $* \mathrm{p}<0.05, * *$ $\mathrm{p}<0.01$.

Figure 4. Ovariectomized females exhibit enhanced cold-induced beiging. Two-month-old female mice underwent sham operation or ovariectomy (OVX), 3 months later both groups were 
either subjected to cold-exposure $\left(6^{\circ} \mathrm{C}\right)$ for 7 days or maintained at $\mathrm{RT}\left(23^{\circ} \mathrm{C}\right)$. (a-c) Representative H\&E-stained histological sections of SWAT (a), VWAT (b) and BAT (c). (d) UCP1 immunohistochemistry - representative sections of SWAT. (e-f) Relative mRNA levels, quantified by qPCR, of brown/beige adipocyte markers expressed in SWAT (IGW) (e) and BAT (f) at cold, $\mathrm{n} \geq 6$. (g) Representative H\&E-stained histological sections of the liver. Arrows indicate fat deposits in OVX at RT. Scale bars $=100 \mu \mathrm{m}$. Error bars indicate S.E.M. Statistical significance assessed by two-tailed student's t-test, $* \mathrm{p}<0.05, * * \mathrm{p}<0.01$.

Figure S4. Related to Figure 4. Two-month-old female mice underwent sham operation or ovariectomy $(\mathrm{OVX}), 3$ months later both groups were either subjected to cold-exposure $\left(6^{\circ} \mathrm{C}\right)$ for 7 days or maintained at RT $\left(23^{\circ} \mathrm{C}\right)$. (a) UCP1 immunohistochemistry - representative sections of VWAT. (b) Relative mRNA levels, quantified by qPCR, of brown/beige adipocyte markers expressed in VWAT (PGW) at cold, $n \geq 10$. (c) Relative mRNA levels, quantified by qPCR, of UCP1 in SWAT (IGW) of Sham and OVX females at RT and cold, $\mathrm{n} \geq 7$. (d) Body temperature (rectal probe) of Sham and OVX females at RT and cold, $\mathrm{n} \geq 11$. (e) Triglyceride levels in the livers of Sham and OVX females at RT and cold, $\mathrm{n} \geq 8$. Scale bars $=100 \mu \mathrm{m}$. Error bars indicate S.E.M. Statistical significance assessed by two-tailed student's t-test, $* \mathrm{p}<0.05, * * \mathrm{p}<0.01$.

Figure 5. Fulvestrant enhances beiging in vitro and in vivo. (a-c) Stromal-vascular (SV) cells were isolated from SWAT of two-month-old females. (a) Confluent cells were treated daily with a vehicle or Fulvestrant for 48hr, and stained for ER $\alpha$ expression, using immunofluorescence. (bc) Confluent cells were induced with beige adipogenic media in the presence of vehicles, Fulvestrant or Estradiol. A week later, beige cells were activated with Forskolin. Beiging was assessed by UCP1 immunostaining (b) or relative mRNA levels, quantified by qPCR, of brown/beige adipocyte markers, $\mathrm{n} \geq 7$ (c). Nile Red stains lipid droplets. (d-j) Acute administration: 
Four-month-old WT females were given a vehicle or $40 \mathrm{mg} / \mathrm{kg} / \mathrm{injection} \mathrm{Fulvestrant} \mathrm{as} \mathrm{described}$ in (d), after they were subjected to cold-exposure $\left(6^{\circ} \mathrm{C}\right)$ for 7 days or maintained at $\mathrm{RT}\left(23^{\circ} \mathrm{C}\right)$. (e) Representative photographs of IGW adipose depots of vehicle-treated and Fulvestrant-treated females at cold. (f) Relative fat mass loss in SWAT and VWAT compartments of vehicle-treated and Fulvestrant-treated females were calculated according to white adipose depot weights in (Fig. S5e): $\quad$ SWAT $=$ Cold $(2 * I G W+I S C W)-R T(2 * I G W+I S C W) ; \quad$ VWAT $=$ Cold $(2 * \mathrm{PGW}+2 * \mathrm{RPW}+\mathrm{MWAT})-\mathrm{RT}(2 * \mathrm{PGW}+2 * \mathrm{RPW}+\mathrm{MWAT}), \mathrm{n} \geq 7$. (g) Representative H\&Estained histological sections of SWAT. (h) UCP1 immunohistochemistry (upper lane) and immunofluorescence (lower lane) - representative sections of SWAT at cold. Arrows indicate $\mathrm{UCP}^{+}$cells. (i-j) Relative mRNA levels, quantified by qPCR, of brown/beige adipocyte markers expressed in SWAT (IGW) (i) and BAT (j) at cold, $n \geq 5$. (k) Vehicle or Fulvestrant administration in four to five-month-old ER $\mathrm{KKO}(\mathrm{KO})$ females in a similar manner to the WT females, as described in (d), after which they were subjected to cold-exposure $\left(6^{\circ} \mathrm{C}\right)$ for 7 days. Relative mRNA levels, quantified by qPCR, of UCP1 in SWAT (IGW) and BAT, $\mathrm{n} \geq 5$. Histological morphology confirms no difference in beiging between vehicle-treated and Fulvestrant-treated KO mice (not shown). Scale bars $=100 \mu \mathrm{m}$. Error bars indicate S.E.M. Statistical significance assessed by two-tailed student's t-test, $* \mathrm{p}<0.05, * * \mathrm{p}<0.01$.

Figure S5. Related to Figure 5. (a-c) SV cells were isolated from SWAT of two-month-old females. (a-b) Confluent cells were induced with white adipogenic media in the presence of vehicle or Fulvestrant. A week later, cells were stained with Oil Red O (a) or immunostained for UCP1 (b). Nile Red stains lipid droplets. Positive control - confluent cells induced with beige adipogenic media for a week, and immunostained for UCP1. (c) Confluent cells were induced with beige adipogenic media in the presence of vehicle or Estradiol. A week later, beige cells were 
activated with Forskolin. Beiging was assessed by relative mRNA levels, quantified by qPCR, of brown/beige adipocyte markers, $n \geq 6$. (d-e) Acute administration: Four-month-old WT females were given a vehicle or $40 \mathrm{mg} / \mathrm{kg} /$ injection Fulvestrant as described in (Fig. 5d), after they were subjected to cold-exposure $\left(6^{\circ} \mathrm{C}\right)$ for 7 days or maintained at RT $\left(23^{\circ} \mathrm{C}\right)$. (d) Body weight, $\mathrm{n} \geq 7$. (e) Weight of indicated fat depots in vehicle-treated and Fulvestrant-treated females: SWAT - IGW

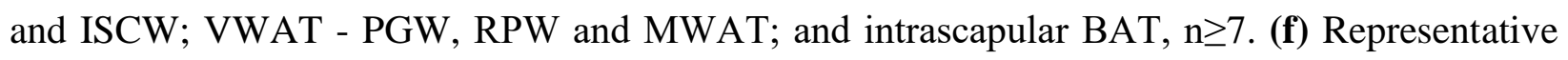
H\&E-stained histological sections of SWAT. Scale bars $=100 \mu \mathrm{m}$. Error bars indicate S.E.M. Statistical significance assessed by two-tailed student's t-test, $* \mathrm{p}<0.05, * * \mathrm{p}<0.01$.

Figure 6. Chronic Fulvestrant treatment reduces adiposity. (a-i) Chronic administration: Fourmonth-old WT ICR(CD1) females were given a vehicle or $40 \mathrm{mg} / \mathrm{kg} /$ injection Fulvestrant for a month as described in (a), after they were subjected to cold-exposure $\left(6^{\circ} \mathrm{C}\right)$ for 7 days or maintained at $\mathrm{RT}\left(23^{\circ} \mathrm{C}\right)$. (b) Body weight, $\mathrm{n} \geq 6$. (c) Fat mass by NMR, $\mathrm{n} \geq 6$. (d) Fat content by NMR, $\mathrm{n} \geq 6$. (e) Representative photographs of IGW and PGW adipose depots of vehicle-treated and Fulvestrant-treated females at cold. (f) Weight of indicated fat depots in vehicle-treated and Fulvestrant-treated females: SWAT - IGW and ISCW; VWAT - PGW, RPW and MWAT; and intrascapular BAT, $\mathrm{n} \geq 6$. (g) Relative fat mass loss in SWAT and VWAT compartments of vehicletreated and Fulvestrant-treated females were calculated according to white adipose depot weights in (f): $\quad$ SWAT $=\operatorname{Cold}(2 * I G W+I S C W)-R T(2 * I G W+I S C W) ; \quad$ VWAT =

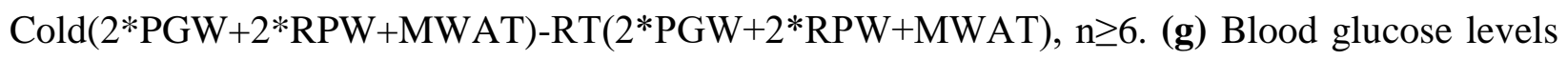
of vehicle-treated and Fulvestrant-treated females, $n \geq 6$. (h) In vivo glucose uptake assay, based on radiolabeled 2-DG administration, in vehicle-treated and Fulvestrant-treated females upon coldexposure (see methods for further details), $\mathrm{n} \geq 5$. Error bars indicate S.E.M. Statistical significance assessed by two-tailed student's t-test, $* \mathrm{p}<0.05, * * \mathrm{p}<0.01, \mathrm{NS}-$ not significant. 
Figure S6. Related to Figure 6. Chronic administration: Four-month-old WT ICR(CD1) females were given a vehicle or $40 \mathrm{mg} / \mathrm{kg} /$ injection Fulvestrant for a month as described in (Fig. 6a), after they were subjected to cold-exposure $\left(6^{\circ} \mathrm{C}\right)$ for 7 days or maintained at RT $\left(23^{\circ} \mathrm{C}\right)$. (a-c) $\mathrm{ER} \alpha$ immunofluorescence of vehicle-treated and Fulvestrant-treated females at RT (prior to coldexposure) - representative sections of SWAT (a), VWAT (b) and BAT (c). Negative controls (lower lane) - representative sections from ER $\alpha$ KO females that lack ER $\alpha$ expression. (d-g) Body weight (d), fat mass by NMR (e), fat content by NMR (f) and lean mass by NMR (g) were monitored during vehicle and Fulvestrant administration and following cold-exposure, $n \geq 11$. (h) Weight of indicated organs in vehicle-treated and Fulvestrant-treated females, $n \geq 6$. Scale bars $=$ $100 \mu \mathrm{m}$. Error bars indicate S.E.M. Statistical significance assessed by two-tailed student's t-test, $* \mathrm{p}<0.05, * * \mathrm{p}<0.01$

\section{Figure 7. Chronic Fulvestrant treatment enhances cold-induced beiging. Chronic} administration: Four-month-old WT ICR(CD1) females were given a vehicle or 40 mg/kg/injection Fulvestrant for a month as described in (Fig. 6a), after they were subjected to coldexposure $\left(6^{\circ} \mathrm{C}\right)$ for 7 days or maintained at RT $\left(23^{\circ} \mathrm{C}\right)$. (a-b) Representative H\&E-stained histological sections of SWAT (a) and VWAT (b). (c-d) UCP1 immunohistochemistry (upper lane) and immunofluorescence (lower lane) - representative sections of SWAT (c) and VWAT (d) at cold. Arrows indicate $\mathrm{UCP}^{+}$cells in vehicle-treated females. (e-g) Relative mRNA levels, quantified by qPCR, of brown/beige adipocyte markers expressed in SWAT (IGW) (e), VWAT (PGW) (f) and BAT (g) at cold, $\mathrm{n} \geq 8$. (h) Body temperature (rectal probe) of vehicle-treated and Fulvestrant-treated females at RT and cold, $\mathrm{n} \geq 6$. Scale bars $=100 \mu \mathrm{m}$. Error bars indicate S.E.M. Statistical significance assessed by two-tailed student's t-test, * $\mathrm{p}<0.05, * * \mathrm{p}<0.01$. 
Figure S7. Related to Figure 7. Chronic administration: Four-month-old WT ICR(CD1) females were given a vehicle or $40 \mathrm{mg} / \mathrm{kg} /$ injection Fulvestrant for a month as described in (Fig. 6a), after they were subjected to cold-exposure $\left(6^{\circ} \mathrm{C}\right)$ for 7 days or maintained at RT $\left(23^{\circ} \mathrm{C}\right)$. (a) Relative mRNA levels, quantified by qPCR, of UCP1 in SWAT (IGW) of vehicle-treated and Fulvestranttreated females at RT and cold, $\mathrm{n} \geq 6$. (d) The body temperatures (rectal probe) of vehicle-treated and Fulvestrant-treated females were monitored upon cold-exposure, $n \geq 12$. Error bars indicate S.E.M. Statistical significance assessed by two-tailed student's t-test, $* \mathrm{p}<0.05, * * \mathrm{p}<0.01$.

Figure 8. Fulvestrant treatment improves the metabolism of diet-induced obese/diabetic mice. (a-k) Chronic administration in a high-fat diet (HFD) model: At the age of two-month-old onwards, WT female mice were fed with high-fat high-sucrose diet. Two months later, the females were given a vehicle or $40 \mathrm{mg} / \mathrm{kg} /$ injection Fulvestrant for a month as described in (a), after they were subjected to cold-exposure $\left(6^{\circ} \mathrm{C}\right)$ for 7 days or maintained at RT $\left(23^{\circ} \mathrm{C}\right)$. (b) Body weight, $n \geq 11$. (c) Fat mass by NMR, $n \geq 17$. (d) Blood glucose levels of vehicle-treated and Fulvestranttreated females on HFD, $n \geq 7$. (e-f) Glucose tolerance (e) and insulin tolerance (f) tests were performed in vehicle-treated and Fulvestrant-treated females on HFD at RT (a week prior to coldexposure) and immediately after cold-exposure. Mice were fasted, i.p. injected with $1.25 \mathrm{~g} / \mathrm{kg}$ glucose (e) or $0.75 \mathrm{U} / \mathrm{kg}$ insulin (f), and their glucose levels were monitored, $\mathrm{n} \geq 8$. Insets - areas under curve. (g) Representative H\&E-stained histological sections of BAT. (h-i) Relative mRNA levels, quantified by qPCR, of brown/beige adipocyte markers expressed in SWAT (IGW) (h), and BAT (i) at cold, $n \geq 6$. (j) Body temperature (rectal probe) of vehicle-treated and Fulvestrant-treated females on HFD at RT and cold, $n \geq 7$. (k) Representative H\&E-stained histological sections of the liver. Arrows indicate fat deposits in females on HFD, particularly vehicle-treated females at RT. 
Scale bars $=100 \mu \mathrm{m}$. Error bars indicate S.E.M. Statistical significance assessed by two-tailed student's t-test, $* \mathrm{p}<0.05, * * \mathrm{p}<0.01$.

Figure S8. Related to Figure 8. (a-l) Chronic administration in a high-fat diet (HFD) model: At the age of two-month-old onwards, WT female mice were fed with high-fat high-sucrose diet. Two months later, the females were given a vehicle or $40 \mathrm{mg} / \mathrm{kg} / \mathrm{injection}$ Fulvestrant for a month as described in (Fig. 8a), after they were subjected to cold-exposure $\left(6^{\circ} \mathrm{C}\right)$ for 7 days or maintained at RT $\left(23^{\circ} \mathrm{C}\right)$. (a) Body weight was monitored from the onset of HFD, during vehicle and Fulvestrant administration and following cold-exposure, $n \geq 21$. (b) Fat content by NMR, $n \geq 17$. (c) Lean mass by NMR, $n \geq 17$. (d) Representative photographs of IGW adipose depots of vehicletreated and Fulvestrant-treated females on HFD at RT and cold. (e) Weight of indicated fat depots in vehicle-treated and Fulvestrant-treated females on HFD: SWAT - IGW and ISCW; VWAT PGW, RPW and MWAT; and intrascapular BAT, $\mathrm{n} \geq 7$. (f) Liver weights of vehicle-treated and Fulvestrant-treated females on HFD, $n \geq 7$. (g) Serum cholesterol and triglyceride levels of vehicletreated and Fulvestrant-treated females on HFD, $\mathrm{n} \geq 7$. (h) Serum insulin levels vehicle-treated and Fulvestrant-treated females on HFD, $\mathrm{n} \geq 7$. (i) Representative histological sections of SWAT H\&E-stained at RT (upper lane) and UCP1 immunofluorescence at cold (lower lane). Arrows indicate $\mathrm{UCP} 1^{+}$cells. (j) Relative mRNA levels, quantified by qPCR, of UCP1 in SWAT (IGW) and BAT of vehicle-treated and Fulvestrant-treated females on HFD at RT and cold, $n \geq 7$. (k) The body temperatures (rectal probe) of vehicle-treated and Fulvestrant-treated females on HFD were monitored upon cold-exposure, $n \geq 7$. (l) Triglyceride levels in the livers of vehicle-treated and Fulvestrant-treated females on HFD at RT and cold, $\mathrm{n} \geq 7$. Scale bars $=100 \mu \mathrm{m}$. Error bars indicate S.E.M. Statistical significance assessed by two-tailed student's t-test, ${ }^{*} \mathrm{p}<0.05, * * \mathrm{p}<0.01$. 
Figure 9. Estrogen receptor inhibition promotes beiging via $\beta 3$-adrenoreceptor. (a-f) SV cells were isolated from SWAT of two-month-old WT females. Confluent cells were induced with beige adipogenic media in the presence of vehicle or Fulvestrant. A week later, the experiments below were performed. (a) Relative changes in cAMP levels upon induction with or without Forskolin. Every dot represents an average of technical triplicates of one biological sample. The relative changes in cAMP levels are calculated as differences between treatments, $n \geq 8$. (b-c) Relative mRNA levels, quantified by qPCR, of UCP1, $\beta$-adrenergic receptors, $\beta$-adrenergic receptor kinases and PPAR $\gamma 2$. (b) Vehicle-treated or Fulvestrant-treated beige cells were activated with Forskolin for $8 \mathrm{hr}, \mathrm{n} \geq 7$. (c) Vehicle or Fulvestrant were not added during differentiation, but rather to mature beige cells for $24 \mathrm{hr}$ without Forskolin, $\mathrm{n} \geq 5$. (d-f) Vehicle or Fulvestrant were added during differentiation. Nile Red stains lipid droplets. (d) Vehicle-treated or Fulvestrant-treated beige cells were immunostained for AdR $\beta 3$ expression. (e) Vehicle-treated or Fulvestrant-treated beige cells were activated or not with CL-316,243 ( $\beta 3$ agonist) for 24hr, and then immunostained for UCP1 expression. (f) Vehicle-treated or Fulvestrant-treated beige cells were activated with Norepinephrine (NE) for 24hr; concomitantly co-treated with a vehicle or SR59230A ( $\beta 3$ antagonist), and then immunostained for UCP1 expression. (g-i) Four-month-old WT females were pre-treated with a vehicle or $40 \mathrm{mg} / \mathrm{kg} /$ injection Fulvestrant, followed by administration of 1 $\mathrm{mg} / \mathrm{kg} /$ day $\beta 3$ agonist as described in $(\mathrm{g})$. Representative histological sections of SWAT, which were either H\&E-stained (h) or immunostained for UCP1 expression (i). (j-k) Four to five-monthold ERaWT or ERaKO (KO) females were treated with $\beta 3$ agonist as described in $(\mathrm{g})$. Representative histological sections of SWAT, which were either H\&E-stained (j) or immunostained for UCP1 expression (k). Arrows indicate UCP1 ${ }^{+}$cells. Scale bars $=100 \mu \mathrm{m}$. Error 
bars indicate S.E.M. Statistical significance assessed by two-tailed student's t-test, * $\mathrm{p}<0.05$, ** $\mathrm{p}<0.01$. In (a), results are based on a matched standard curve and a linear regression analysis.

Figure S9. Related to Figure 9. (a-e) SV cells were isolated from SWAT of two-month-old WT females. Undifferentiated cells: confluent cells were treated daily with a vehicle or Fulvestrant for 8-48hr, in the absence of beige adipogenic reagents (a-e). Beige cells: Confluent cells were induced with beige adipogenic media in the presence of vehicle or Fulvestrant for a week (c). (a) Relative changes in cAMP levels upon induction with or without Forskolin. Every dot represents an average of technical triplicates of one biological sample. The relative changes in cAMP levels are calculated as differences between treatments, $\mathrm{n} \geq 7$. (b, d) Relative mRNA levels, quantified by qPCR, of UCP1, $\beta$-adrenergic receptors, $\beta$-adrenergic receptor kinases and PPAR $\gamma 2, \mathrm{n} \geq 5$. (c, e) Vehicle-treated or Fulvestrant-treated undifferentiated cells were immunostained for AdR $\beta 3$ (c) or PCNA (e) expression. Arrows indicate $\mathrm{PCNA}^{+}$cells and the numbers represent their percentage. (f) Confluent cells were treated daily with a vehicle or Fulvestrant for 48hr, and then induced with beige adipogenic media in the presence of $\beta 3$ agonist without Fulvestrant. Beiging was assessed by UCP1 immunostaining. Nile Red stains lipid droplets. (g-h) Vehicle-treated and Fulvestranttreated females (g, see Fig. 5) as well as ER $\alpha$ WT (WT) and ER $\alpha$ KO (KO) females (h, see Fig. 12) were subjected to cold-exposure $\left(6^{\circ} \mathrm{C}\right)$ for 7 days or maintained at RT $\left(23^{\circ} \mathrm{C}\right)$. Representative histological sections of SWAT, which were immunostained for AdR $\beta 3$ and Perilipin. Arrows indicate $\mathrm{AdR}_{3} 3^{+}$adipocytes in Fulvestrant-treated females at RT, KO females at RT, and all specimens at cold. (i-k) Four-month-old WT females were pre-treated with a vehicle or 40 $\mathrm{mg} / \mathrm{kg} /$ injection Fulvestrant, followed by administration of $1 \mathrm{mg} / \mathrm{kg} /$ day $\beta 3$ agonist as described in (Fig. 9g). (i) Relative fat mass loss in SWAT and VWAT compartments were calculated according to white adipose depot weights (not shown), $n \geq 8$. (j-k) Relative mRNA levels, 
quantified by qPCR, of brown/beige adipocyte markers expressed in SWAT (IGW) (j), and BAT (k), $\mathrm{n} \geq 7$. Scale bars $=100 \mu \mathrm{m}$. Error bars indicate S.E.M. Statistical significance assessed by twotailed student's t-test, $* \mathrm{p}<0.05, * * \mathrm{p}<0.01$. In (a), results are based on a matched standard curve and a linear regression analysis.

Figure 10. Summary. (a) In this study, we studied mouse models of inhibited ER $\alpha$ signaling: 1) a genetic approach - whole-body ER $\alpha \mathrm{KO}$ mice; 2) a surgical approach - OVX females; 3) a pharmacological approach - treatment with the estrogen receptor antagonist - Fulvestrant, and exposed them to cold. Upon cold-exposure, we detected adipocyte beiging in control mice (e.g. WT, Sham or vehicle-treated) as expected, however, mouse models of inhibited ER $\alpha$ showed an enhanced response. This response included increased emergence of multilocular beige cells and elevated gene expression of beige/brown adipocyte markers in WAT and BAT. By using a primary beige cell culture, we demonstrated that this enhanced beiging is cell-autonomous. (b) Although under normal circumstances of room temperature, ER $\alpha \mathrm{KO}, \mathrm{OVX}$ and mice on HFD are obese and diabetic, cold-exposure resulted in significantly improved metabolism. As a result of coldexposure, we observed: reduced adiposity and hyperglycemia, increased glucose and insulin sensitivities, and corrected diabetes-associated hypercholesterolemia, hypertriglyceridemia and hepatosteatosis. (c) Hypothesis - estrogen receptor inhibition enhances adipocyte beiging by "sensitizing" the cells to the beiging process upon stimulation. Stimuli of beiging may be elicited by cold-exposure or adrenergic signals. Our preliminary results support such a hypothesis Fulvestrant pre-treatment increased cAMP levels and AdR $\beta 3$ expression in both immature and mature cells. This allowed increased beige cell differentiation or activation via AdR $\beta 3$. The mechanism that underlies enhanced cold-induced adipocyte beiging following ER $\alpha$ inhibition is yet to be elucidated. 
Supplementary Table 1. qPCR primer sequences.

\begin{tabular}{|c|c|c|}
\hline Gene & Forward primer sequence & Reverse primer sequence \\
\hline$A \cot 2$ & ATGGTGGCCTCGTCTTTCG & GAGCGGCGGAGGTACAAAC \\
\hline $\operatorname{Adr} \beta 1$ & CTCGTCCGTCGTCTCCTTCTAC & GTCGATCTTCTTTACCTGTTTTTGG \\
\hline $\operatorname{Adr} \beta 2$ & TTGCAGTGGATCGCTATGTTG & TGACCACTCGGGCCTTATTCT \\
\hline $\operatorname{Adr} \beta 3$ & GGCCCTCTCTAGTTCCCAG & TAGCCATCAAACCTGTTGAGC \\
\hline Adr $\beta \mathrm{k} 1$ & AGCGAGTACCCAAGATGAAGAACA & CACTGCCACGCTGGATCA \\
\hline Adr $\beta k 2$ & GGACAGAAGTCGTTACAGCTAATTCA & ACTCCCAAGCTGCTGTCAAA \\
\hline Cidea & TGCTCTTCTGTATCGCCCAGT & GCCGTGTTAAGGAATCTGCTG \\
\hline Cox7a1 & CAGCGTCATGGTCAGTCTGT & AGAAAACCGTGTGGCAGAGA \\
\hline$\beta$-Actin & GCGCATCCTCTTCCT & GGAGCAATGATCTTGATC \\
\hline Eva1 & CCACTTCTCCTGAGTTTACAGC & GCATTTTAACCGAACATCTGTCC \\
\hline Oplah & CTTCACGCACGTCTCCTTGT & GCATCTGCACAGGCCGTAT \\
\hline PGC1 $\alpha$ & AGCCGTGACCACTGACAACGAG & GCTGCATGGTTCTGAGTGCTAAG \\
\hline PPAR $\gamma 2$ & ACAGCAAATCTCTGTTTATGC & GCTTGATGTCAAAGGAATGCG \\
\hline PRDM16 & CAGCACGGTGAAGCCATTC & GCGTGCATCCGCTTGTG \\
\hline Slc29a1 & CACCAGCCTCAGGACAGGTAT & GTCCAGGCGGTTTGTGAAA \\
\hline UCP1 & ACTGCCACACCTCCAGTCATT & CTTTGCCTCACTCAGGATTGG \\
\hline
\end{tabular}

\section{References}

1 Lovre, D., Lindsey, S. H. \& Mauvais-Jarvis, F. Effect of menopausal hormone therapy on components of the metabolic syndrome. Ther Adv Cardiovasc Dis, doi:10.1177/1753944716649358 (2016).

2 Palmer, B. F. \& Clegg, D. J. The sexual dimorphism of obesity. Mol Cell Endocrinol 402, 113-119, doi:10.1016/j.mce.2014.11.029 (2015).

3 Heine, P. A., Taylor, J. A., Iwamoto, G. A., Lubahn, D. B. \& Cooke, P. S. Increased adipose tissue in male and female estrogen receptor-alpha knockout mice. Proc Natl Acad Sci U S A 97, 1272912734, doi:10.1073/pnas.97.23.12729 (2000).

4 Pallier, E., Aubert, R. \& Lemonnier, D. Effect of diet and ovariectomy on adipose tissue cellularity in mice. Reprod Nutr Dev 20, 631-636 (1980).

5 Stubbins, R. E., Holcomb, V. B., Hong, J. \& Nunez, N. P. Estrogen modulates abdominal adiposity and protects female mice from obesity and impaired glucose tolerance. Eur J Nutr 51, 861-870, doi:10.1007/s00394-011-0266-4 (2012).

6 Stefanick, M. L. Postmenopausal hormone therapy and cardiovascular disease in women. Nutr Metab Cardiovasc Dis 20, 451-458, doi:10.1016/j.numecd.2010.02.015 (2010).

7 Rozenberg, S., Vandromme, J. \& Antoine, C. Postmenopausal hormone therapy: risks and benefits. Nat Rev Endocrinol 9, 216-227, doi:10.1038/nrendo.2013.17 (2013). 
8 Lapid, K., Lim, A., Clegg, D. J., Zeve, D. \& Graff, J. M. Oestrogen signalling in white adipose progenitor cells inhibits differentiation into brown adipose and smooth muscle cells. Nat Commun 5, 5196, doi:10.1038/ncomms6196 (2014).

9 Townsend, K. L. \& Tseng, Y. H. Of mice and men: novel insights regarding constitutive and recruitable brown adipocytes. Int J Obes Supp/ 5, S15-20, doi:10.1038/ijosup.2015.5 (2015). Thyagarajan, B. \& Foster, M. T. Beiging of white adipose tissue as a therapeutic strategy for weight loss in humans. Horm Mol Biol Clin Investig 31, doi:10.1515/hmbci-2017-0016 (2017). Kim, S. H. \& Plutzky, J. Brown Fat and Browning for the Treatment of Obesity and Related Metabolic Disorders. Diabetes Metab J 40, 12-21, doi:10.4093/dmj.2016.40.1.12 (2016).

12 Stanford, K. I. et al. Brown adipose tissue regulates glucose homeostasis and insulin sensitivity. J Clin Invest 123, 215-223, doi:10.1172/JCI62308 (2013).

13 Wang, W. \& Seale, P. Control of brown and beige fat development. Nat Rev Mol Cell Biol 17, 691-702, doi:10.1038/nrm.2016.96 (2016).

14 Hanssen, M. J. et al. Short-term cold acclimation improves insulin sensitivity in patients with type 2 diabetes mellitus. Nat Med 21, 863-865, doi:10.1038/nm.3891 (2015).

15 Vallerand, A. L., Lupien, J. \& Bukowiecki, L. J. Cold exposure reverses the diabetogenic effects of high-fat feeding. Diabetes 35, 329-334 (1986).

16 Bartelt, A. et al. Brown adipose tissue activity controls triglyceride clearance. Nat Med 17, 200205, doi:10.1038/nm.2297 (2011).

17 Johnston, S. J. \& Cheung, K. L. Fulvestrant - a novel endocrine therapy for breast cancer. Curr Med Chem 17, 902-914 (2010).

18 Davis, K. E. et al. The sexually dimorphic role of adipose and adipocyte estrogen receptors in modulating adipose tissue expansion, inflammation, and fibrosis. Mol Metab 2, 227-242, doi:10.1016/j.molmet.2013.05.006 (2013).

19 Berglund, E. D. et al. Glucose metabolism in vivo in four commonly used inbred mouse strains. Diabetes 57, 1790-1799, doi:10.2337/db07-1615 (2008).

20 Berry, D. C. et al. Cellular Aging Contributes to Failure of Cold-Induced Beige Adipocyte Formation in Old Mice and Humans. Cell Metab 25, 166-181, doi:10.1016/j.cmet.2016.10.023 (2017).

21 Tang, W. et al. White fat progenitor cells reside in the adipose vasculature. Science 322, 583586, doi:10.1126/science.1156232 (2008).

22 Ribas, V. et al. Impaired oxidative metabolism and inflammation are associated with insulin resistance in ERalpha-deficient mice. Am J Physiol Endocrinol Metab 298, E304-319, doi:10.1152/ajpendo.00504.2009 (2010).

23 Rabii, J. \& Ganong, W. F. Responses of plasma "estradiol" and plasma LH to ovariectomy, ovariectomy plus adrenalectomy, and estrogen injection at various ages. Neuroendocrinology 20, 270-281 (1976).

24 Vieira Potter, V. J. et al. Adipose tissue inflammation and reduced insulin sensitivity in ovariectomized mice occurs in the absence of increased adiposity. Endocrinology 153, 4266 4277, doi:10.1210/en.2011-2006 (2012).

25 Zhu, L. et al. Estrogen treatment after ovariectomy protects against fatty liver and may improve pathway-selective insulin resistance. Diabetes 62, 424-434, doi:10.2337/db11-1718 (2013).

26 Wade, G. N., Blaustein, J. D., Gray, J. M. \& Meredith, J. M. ICl 182,780: a pure antiestrogen that affects behaviors and energy balance in rats without acting in the brain. Am J Physiol 265, R1392-1398 (1993).

27 Pink, J. J. \& Jordan, V. C. Models of estrogen receptor regulation by estrogens and antiestrogens in breast cancer cell lines. Cancer Res 56, 2321-2330 (1996). 

novel, "pure" antiestrogen. Cancer 89, 817-825 (2000).

29 Eaton, G. J., Johnson, F. N., Custer, R. P. \& Crane, A. R. The Icr:Ha(ICR) mouse: a current account of breeding, mutations, diseases and mortality. Lab Anim 14, 17-24, doi:10.1258/002367780780943141 (1980).

30 Robertson, J. F., Odling-Smee, W., Holcombe, C., Kohlhardt, S. R. \& Harrison, M. P. Pharmacokinetics of a single dose of fulvestrant prolonged-release intramuscular injection in postmenopausal women awaiting surgery for primary breast cancer. Clin Ther 25, 1440-1452 (2003).

31 Shi, F. \& Collins, S. Second messenger signaling mechanisms of the brown adipocyte thermogenic program: an integrative perspective. Horm Mol Biol Clin Investig 31, doi:10.1515/hmbci-2017-0062 (2017).

32 Tontonoz, P., Hu, E. \& Spiegelman, B. M. Stimulation of adipogenesis in fibroblasts by PPAR gamma 2, a lipid-activated transcription factor. Cell 79, 1147-1156 (1994).

33 Chawla, A., Schwarz, E. J., Dimaculangan, D. D. \& Lazar, M. A. Peroxisome proliferator-activated receptor (PPAR) gamma: adipose-predominant expression and induction early in adipocyte differentiation. Endocrinology 135, 798-800, doi:10.1210/endo.135.2.8033830 (1994).

34 Berry, D. C., Jiang, Y. \& Graff, J. M. Mouse strains to study cold-inducible beige progenitors and beige adipocyte formation and function. Nat Commun 7, 10184, doi:10.1038/ncomms10184 (2016). brown fat conversion through stabilization of PRDM16 protein. Cell Metab 15, 395-404, doi:10.1016/j.cmet.2012.01.019 (2012).

36 Qiu, S. et al. Hepatic estrogen receptor alpha is critical for regulation of gluconeogenesis and lipid metabolism in males. Sci Rep 7, 1661, doi:10.1038/s41598-017-01937-4 (2017).

37 Ribas, V. et al. Skeletal muscle action of estrogen receptor alpha is critical for the maintenance of mitochondrial function and metabolic homeostasis in females. Sci Transl Med 8, 334ra354, doi:10.1126/scitranslmed.aad3815 (2016).

$38 \mathrm{Xu}, \mathrm{Y}$. et al. Distinct hypothalamic neurons mediate estrogenic effects on energy homeostasis and reproduction. Cell Metab 14, 453-465, doi:10.1016/j.cmet.2011.08.009 (2011).

$39 \mathrm{Xu}, \mathrm{P}$. et al. Estrogen receptor-alpha in medial amygdala neurons regulates body weight. J Clin Invest 125, 2861-2876, doi:10.1172/JCl80941 (2015).

40 Xu, Y., O'Malley, B. W. \& Elmquist, J. K. Brain nuclear receptors and body weight regulation. J Clin Invest 127, 1172-1180, doi:10.1172/JCl88891 (2017).

41 Martinez de Morentin, P. B. et al. Estradiol regulates brown adipose tissue thermogenesis via hypothalamic AMPK. Cell Metab 20, 41-53, doi:10.1016/j.cmet.2014.03.031 (2014).

42 Cousin, B. et al. Occurrence of brown adipocytes in rat white adipose tissue: molecular and morphological characterization. J Cell Sci 103 ( Pt 4), 931-942 (1992).

43 de Jong, J. M. A. et al. The beta3-adrenergic receptor is dispensable for browning of adipose tissues. Am J Physiol Endocrinol Metab 312, E508-E518, doi:10.1152/ajpendo.00437.2016 (2017).

44 Jiang, Y., Berry, D. C. \& Graff, J. M. Distinct cellular and molecular mechanisms for beta3 adrenergic receptor-induced beige adipocyte formation. Elife 6, doi:10.7554/eLife.30329 (2017). 
Fig. 1

a

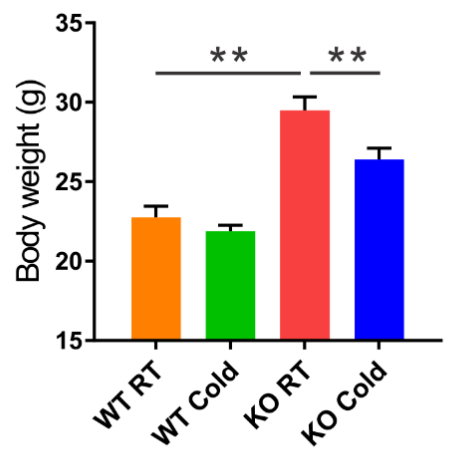

b

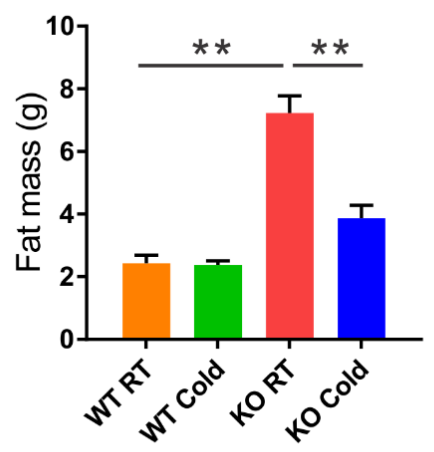

C

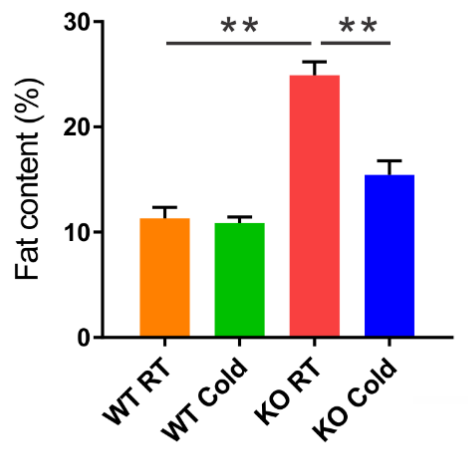

d

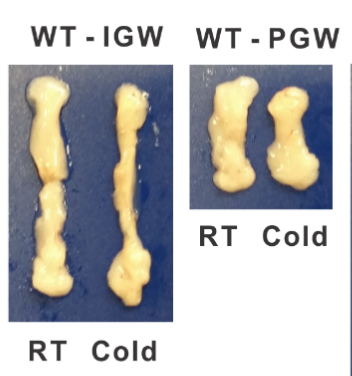

f

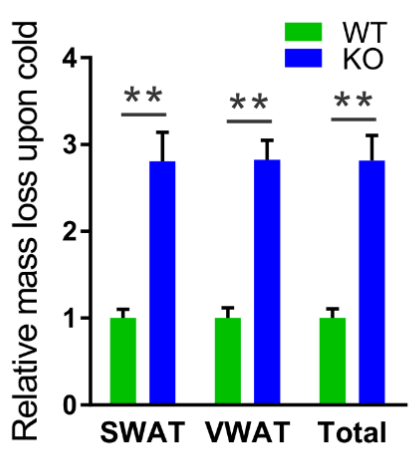

g e

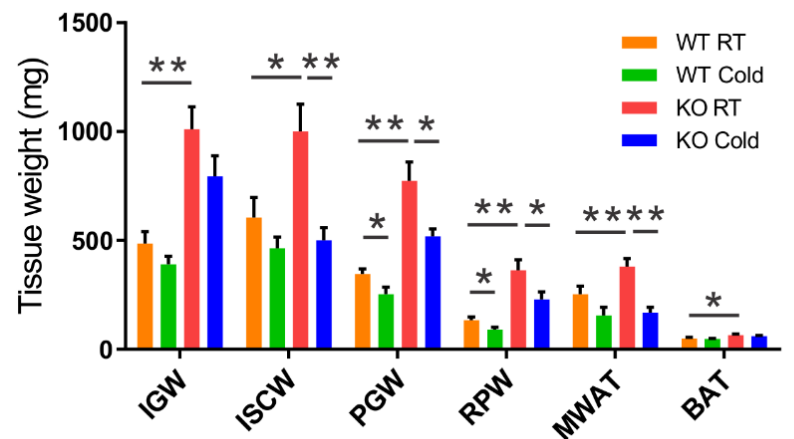

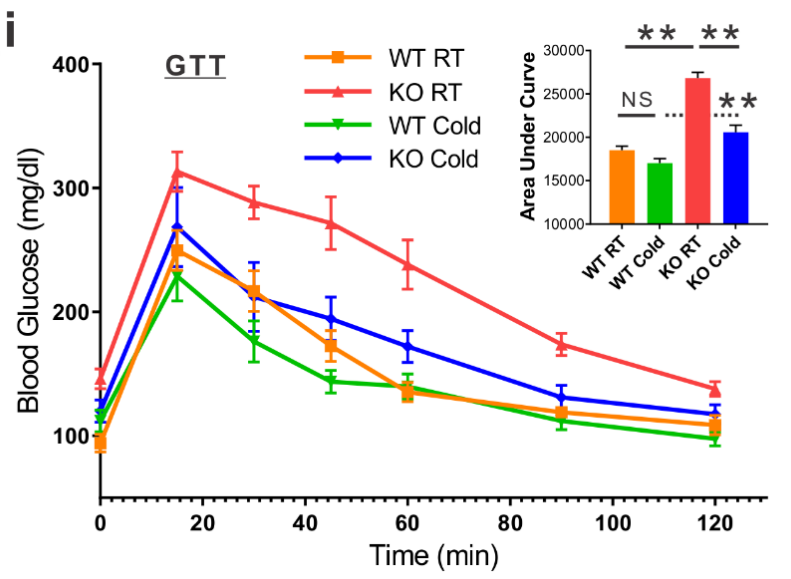

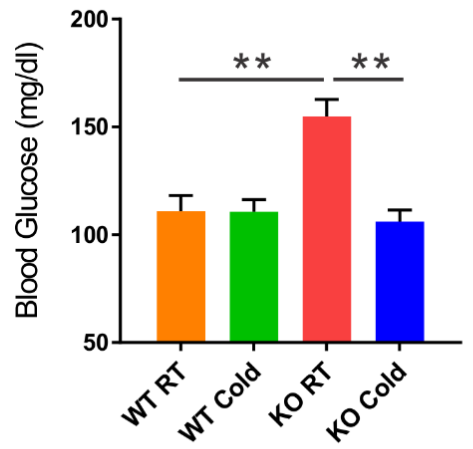

h
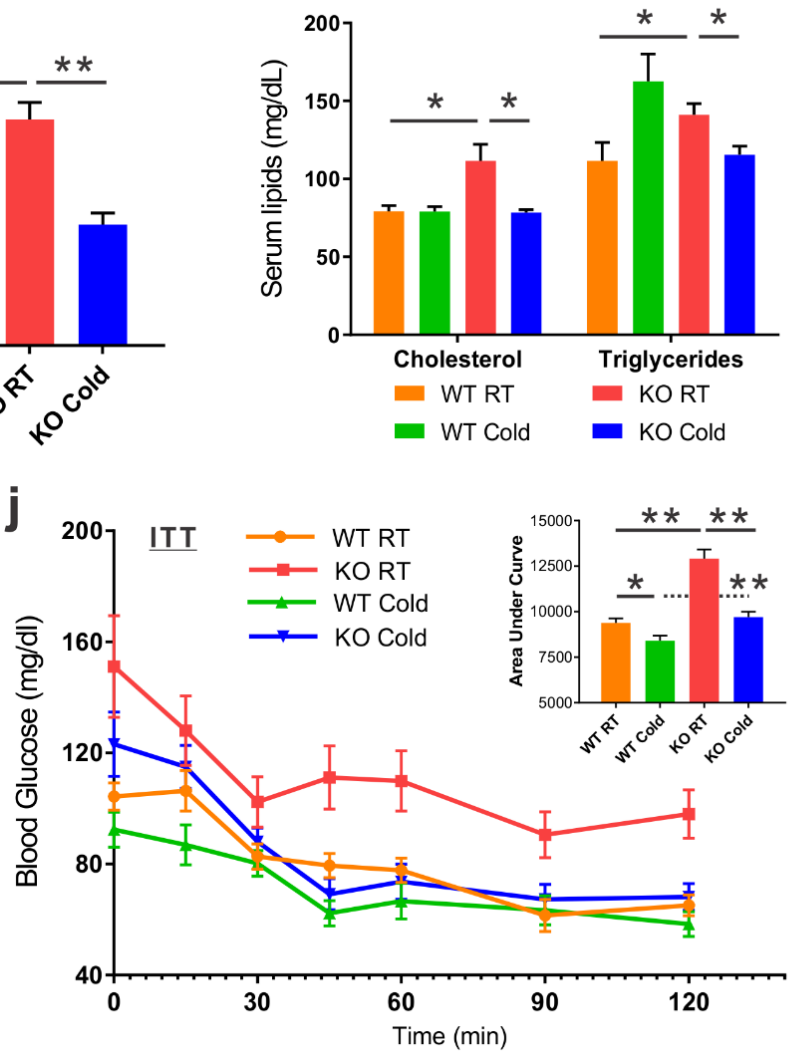


\section{Fig. S1}

a

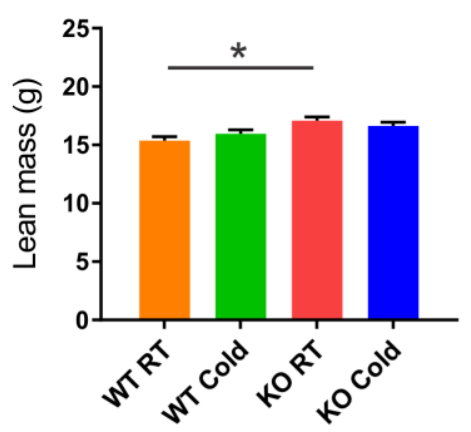

b

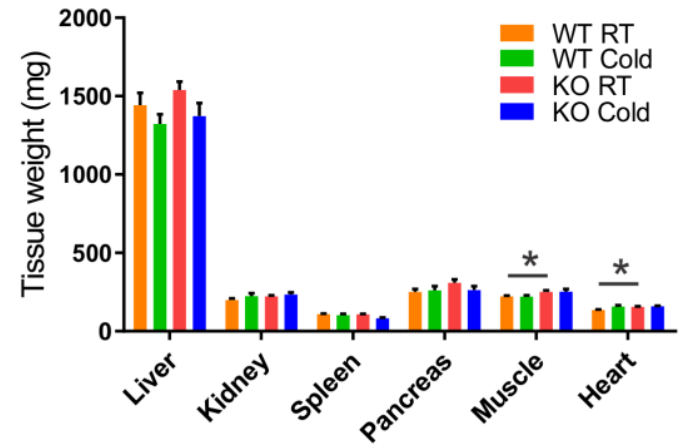

C

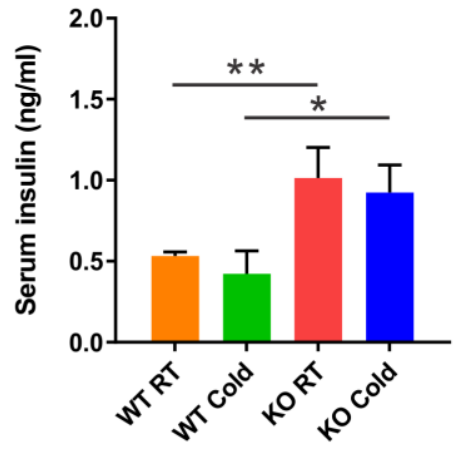




\section{Fig. 2}

a

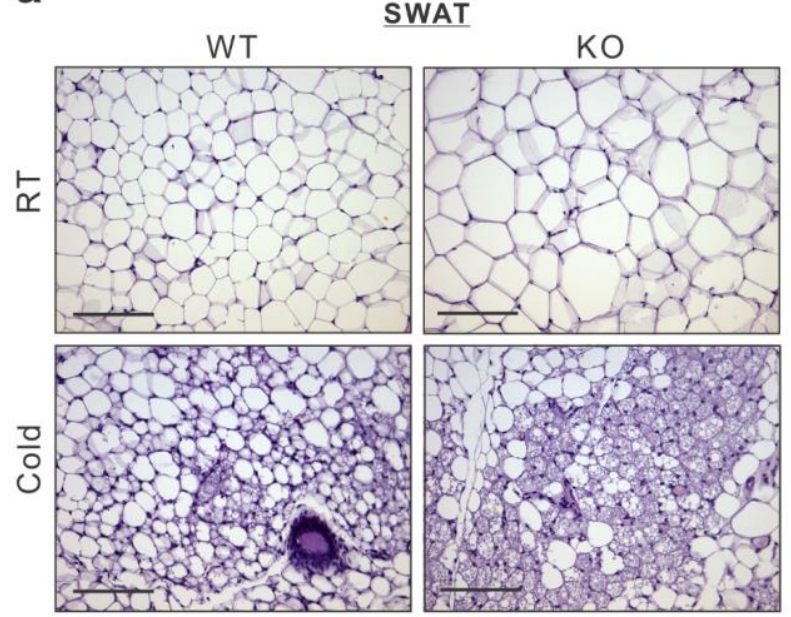

C

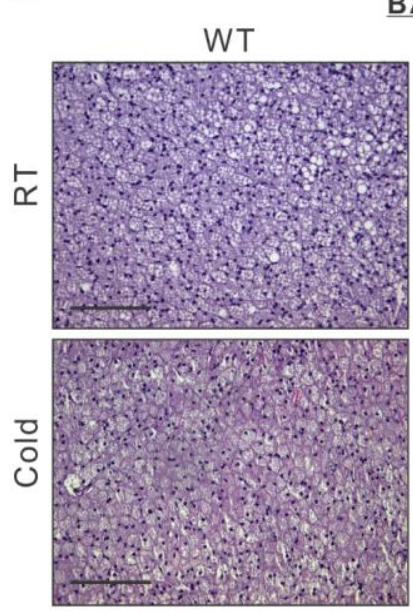

e

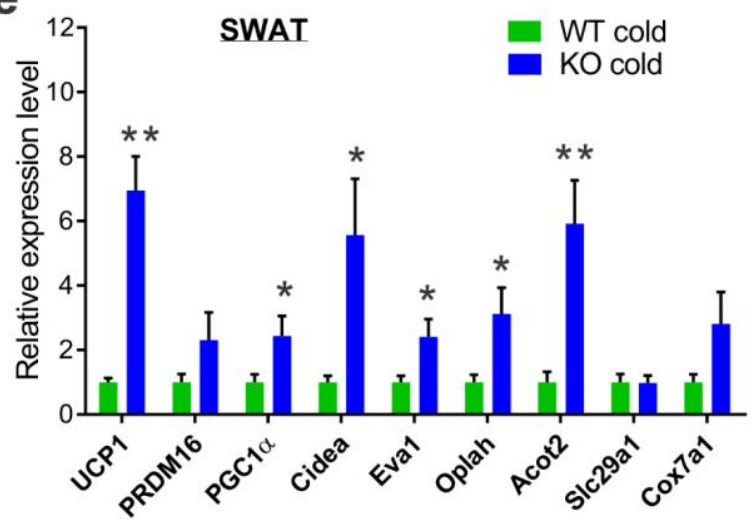

b

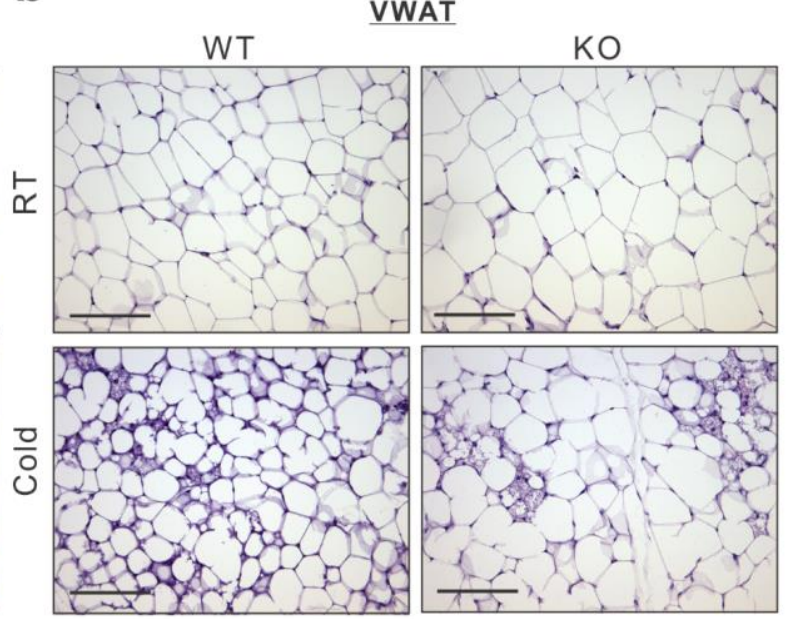

d
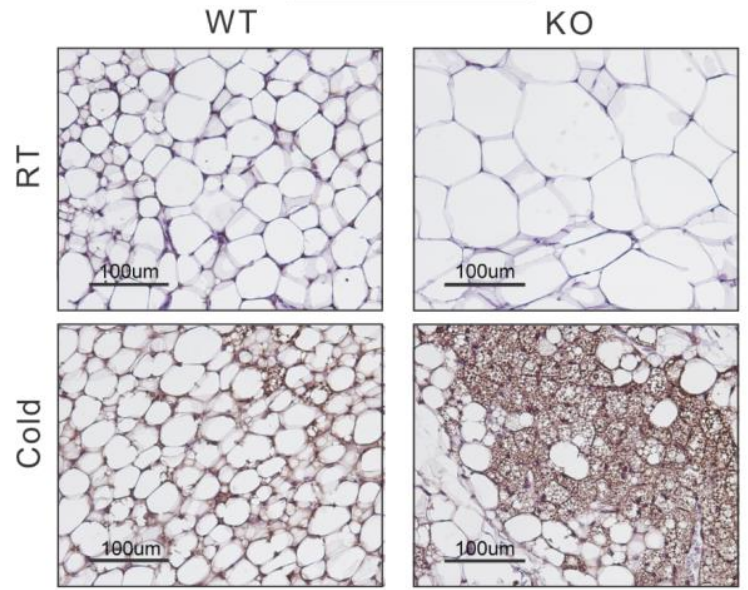

f

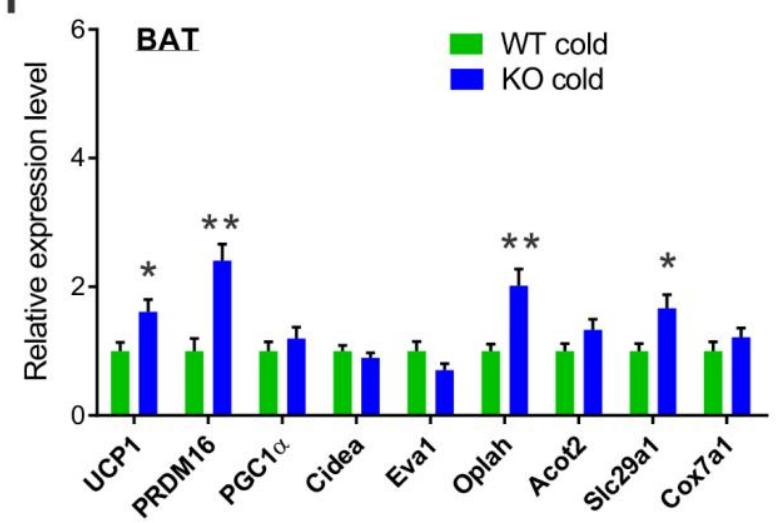



under aCC-BY-NC-ND 4.0 International license.

\section{Fig. S2}

a

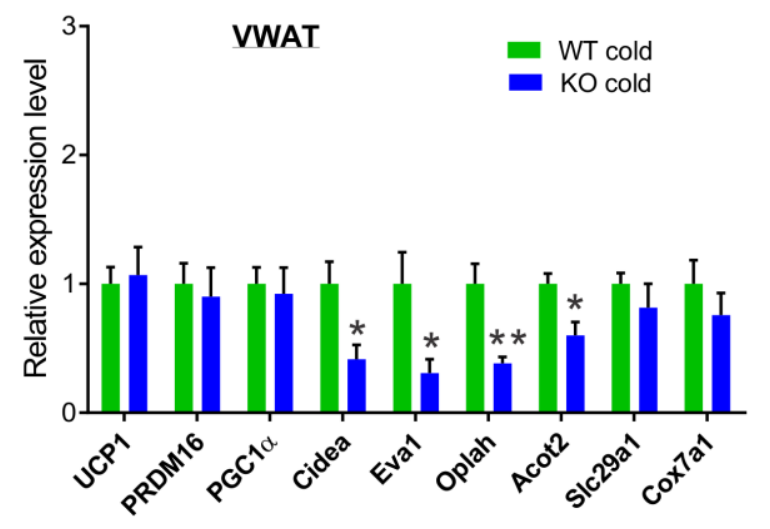

b

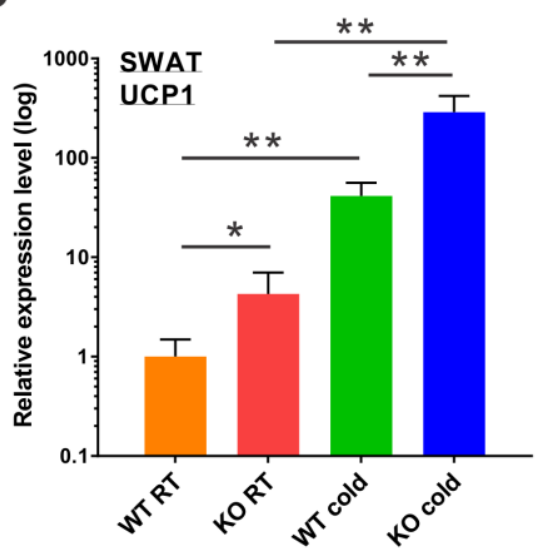

C

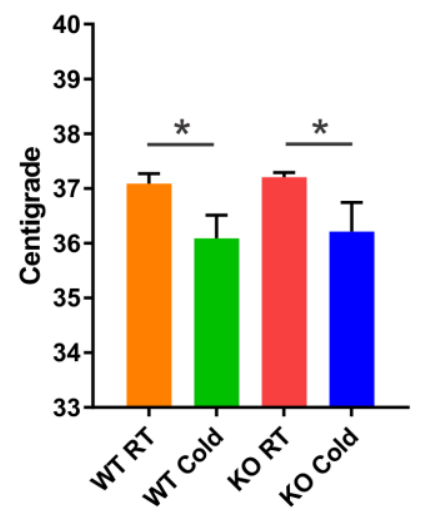


Fig. 3

a

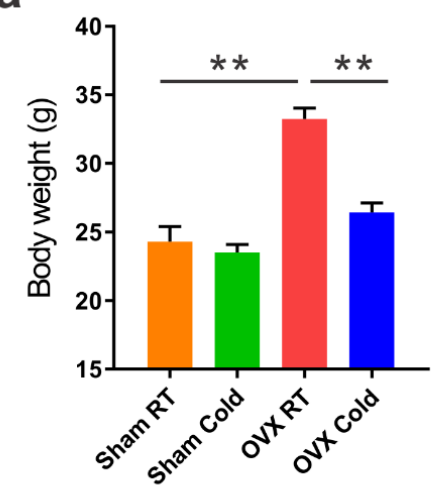

b

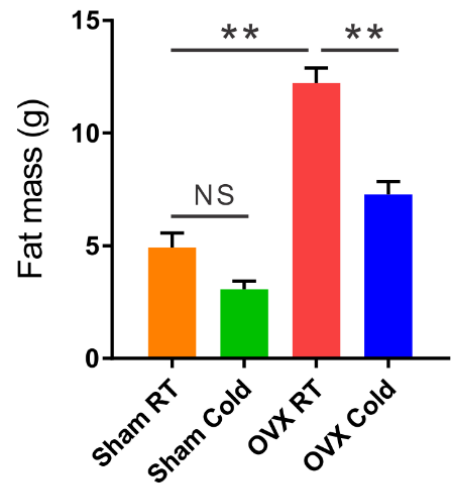

C

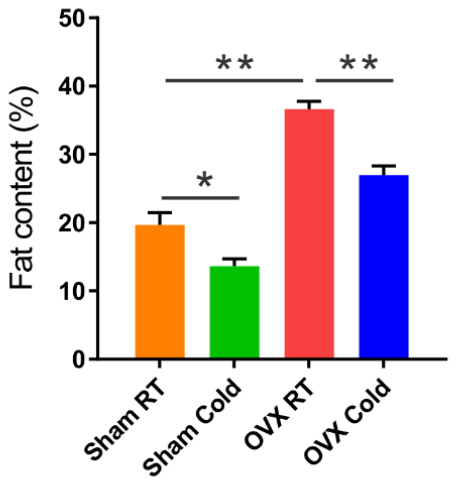

d

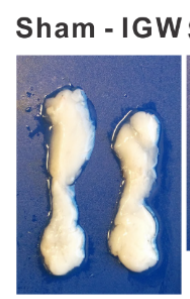

RT Cold
RT Cold

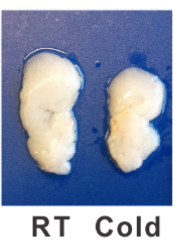

RT Cold

e
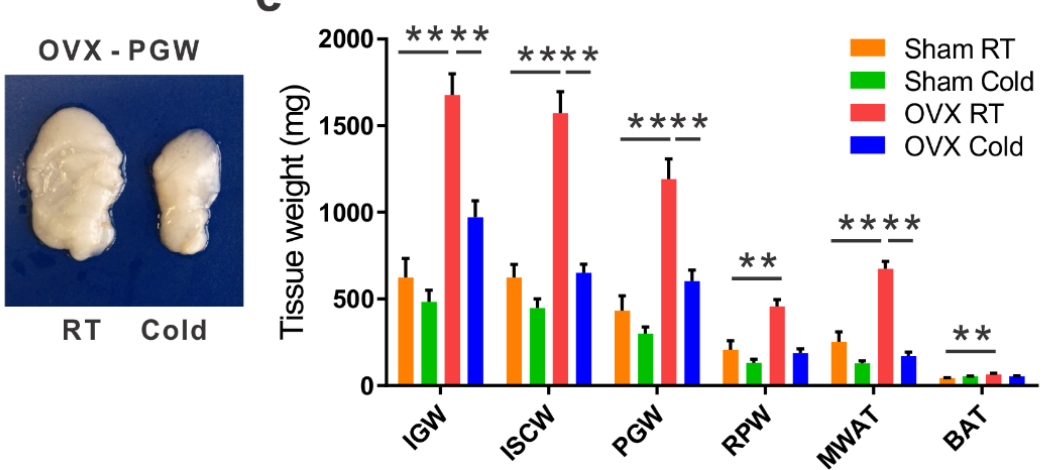

f

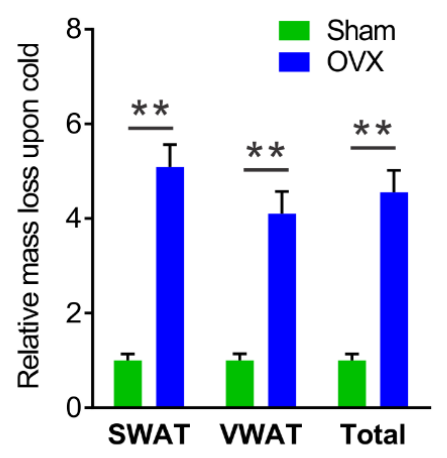

i

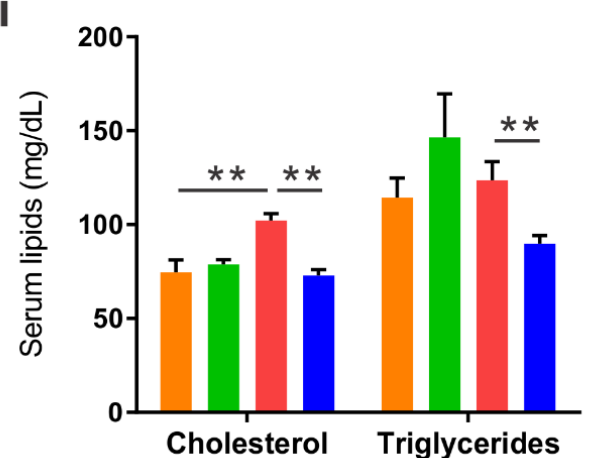

- Sham RT OVXRT

- Sham Cold g

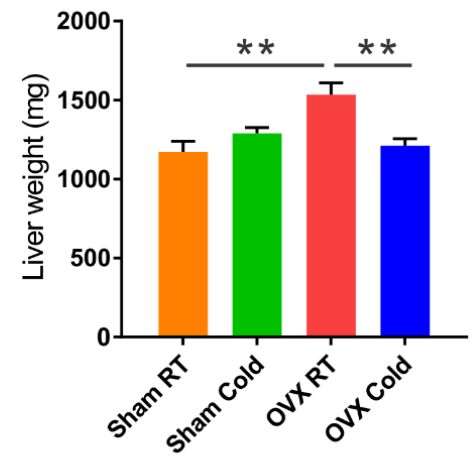

h

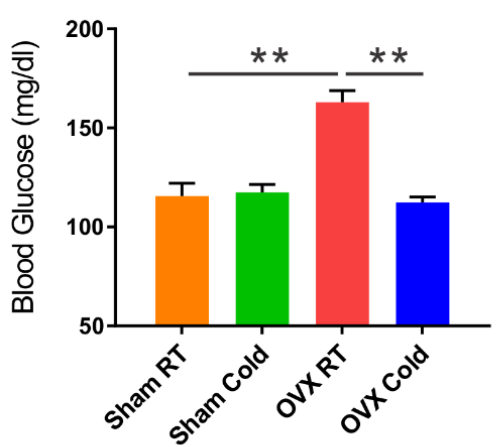

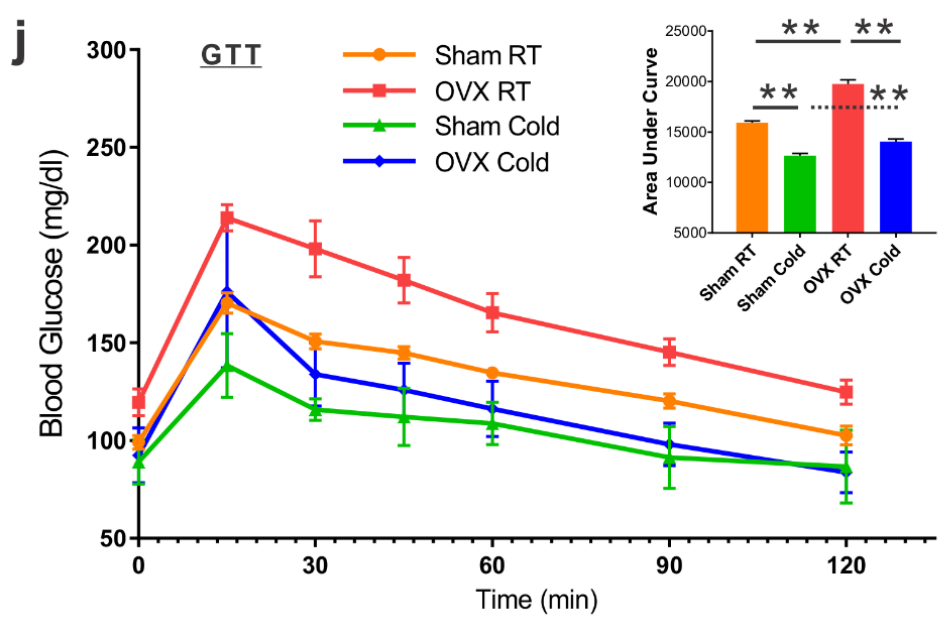




\section{Fig. S3}

a

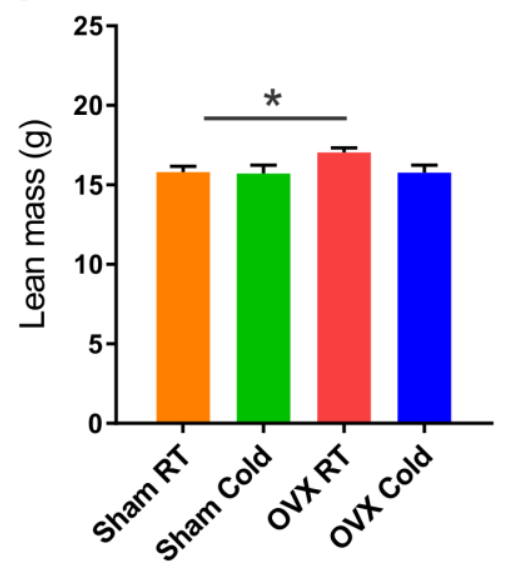

C

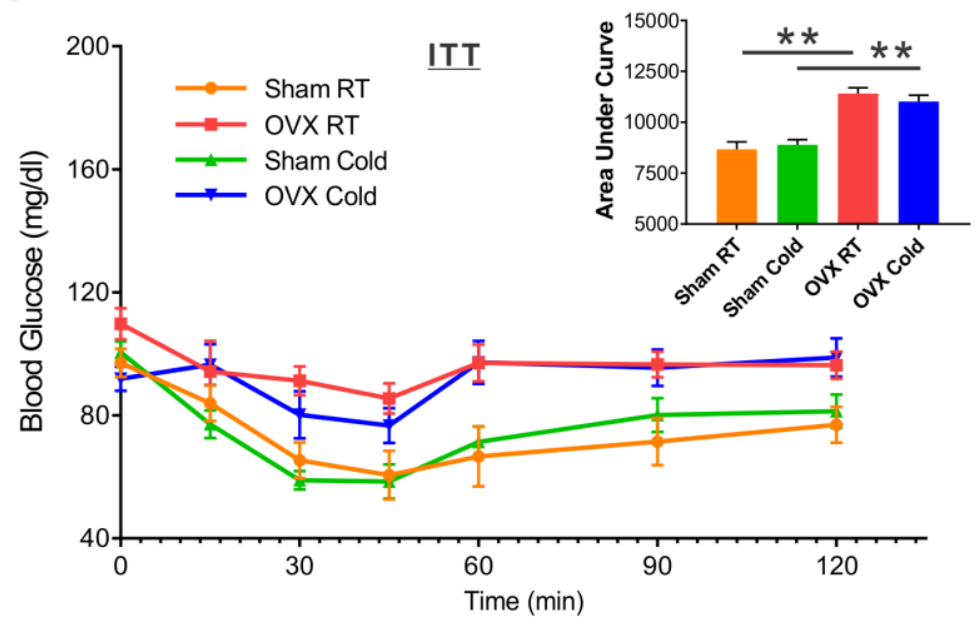

b

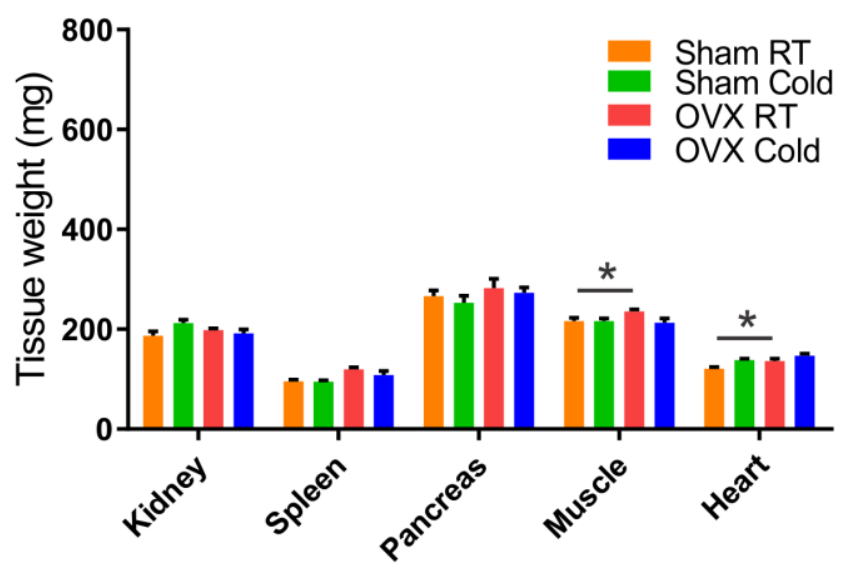

d

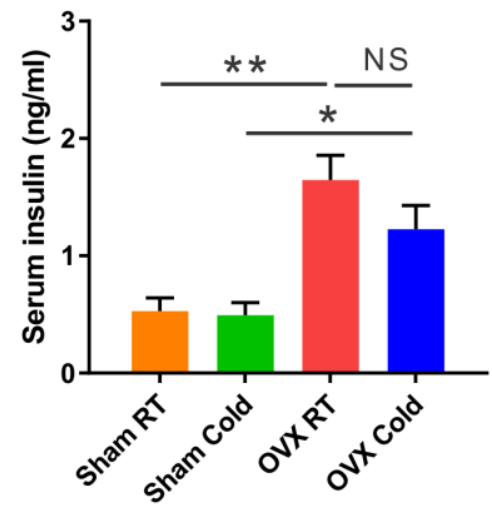




\section{Fig. 4}

a

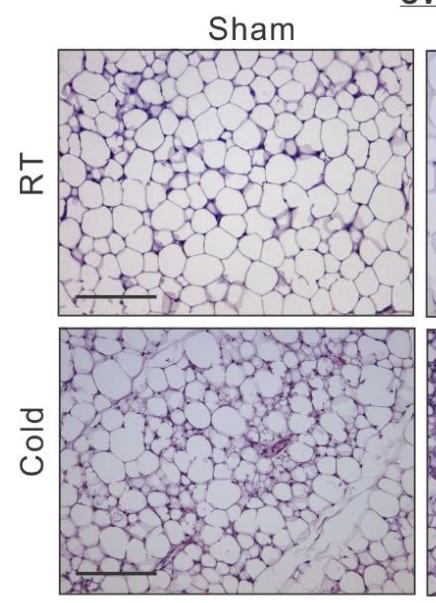

C

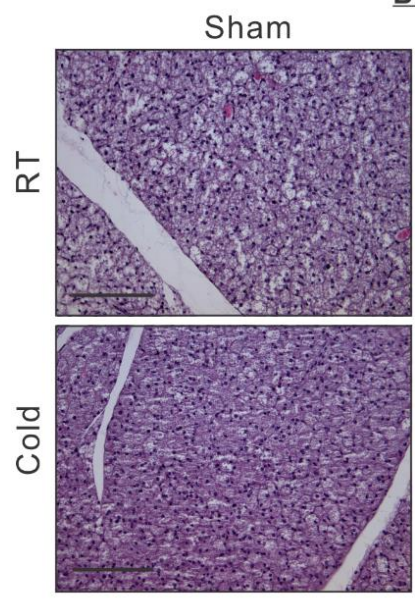

e

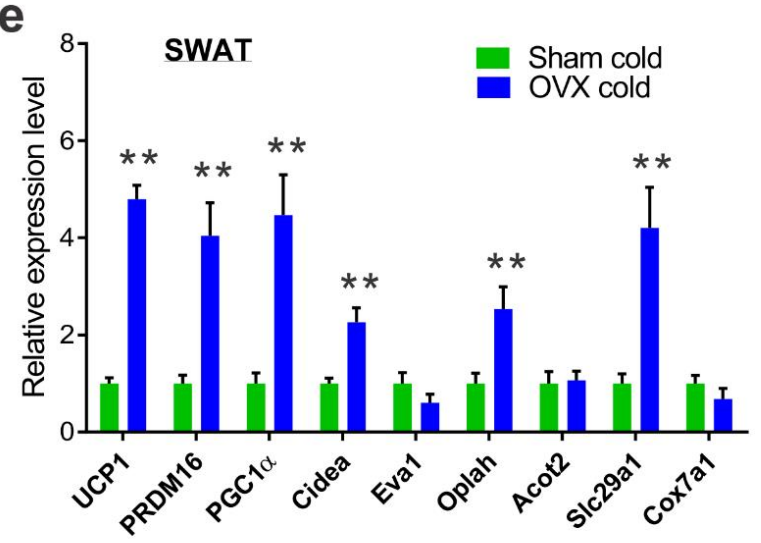

g

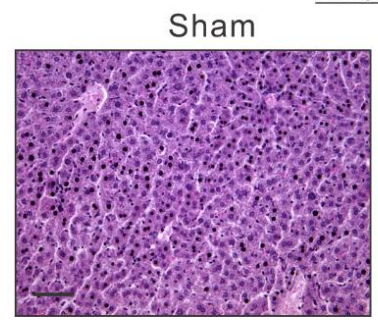

SWAT

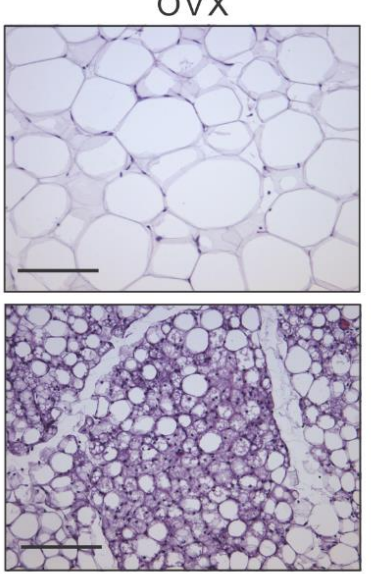

b

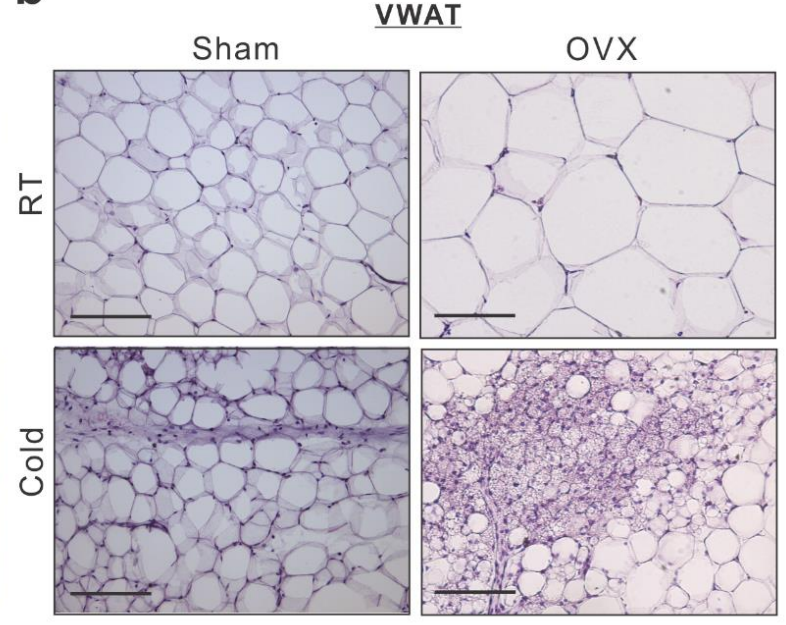

d

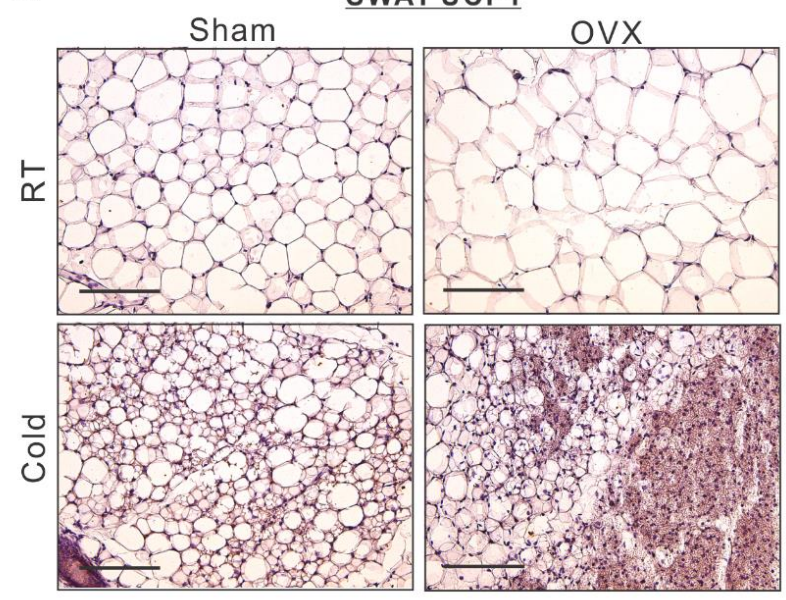

f

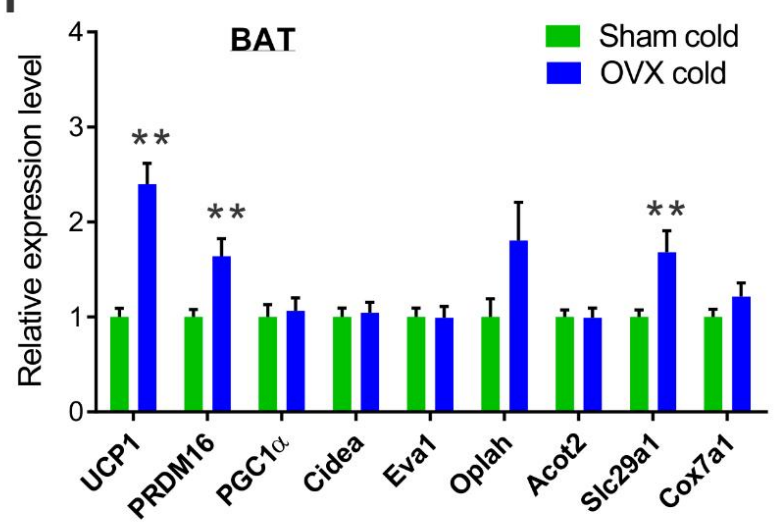

Liver - Cold

Sham

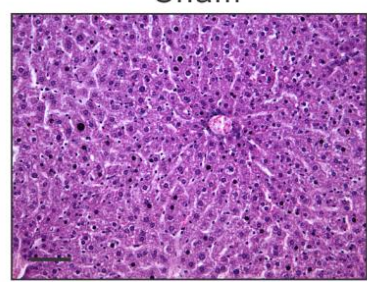

OVX

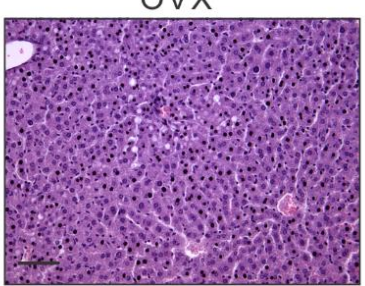




\section{Fig. S4}

a

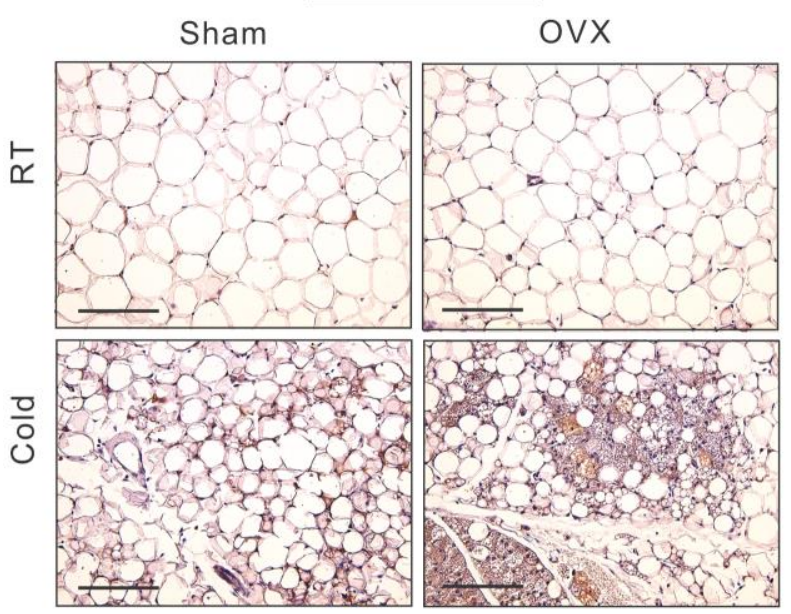

C

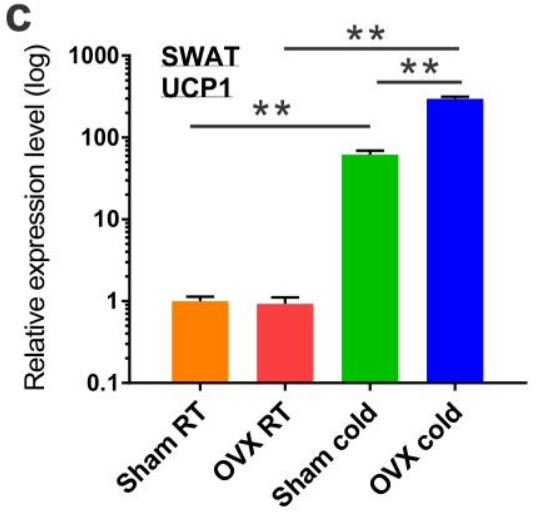

d

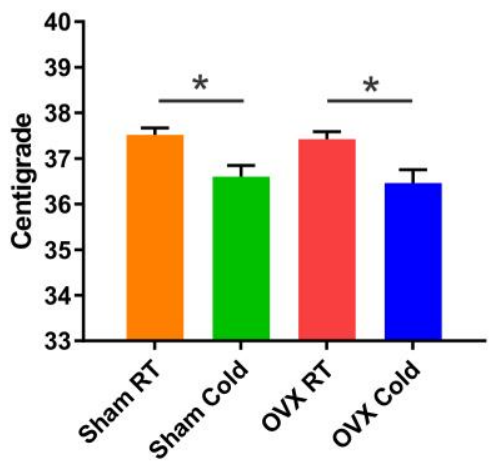

VWAT

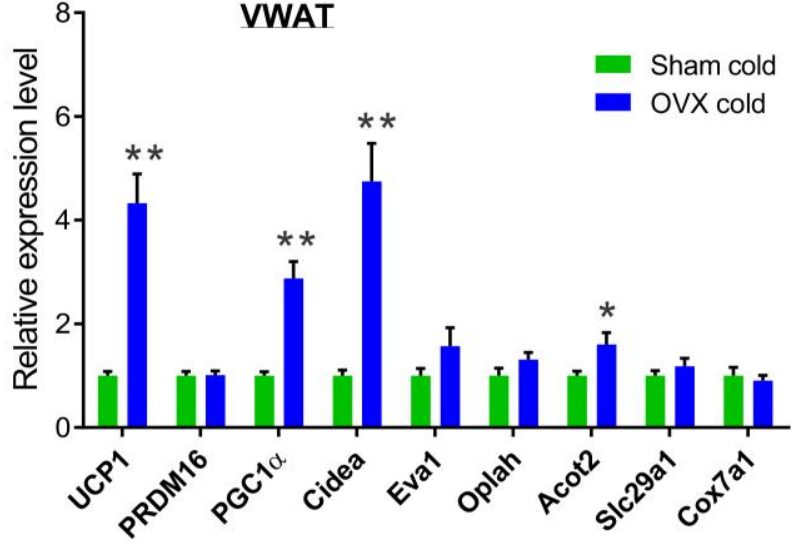

e

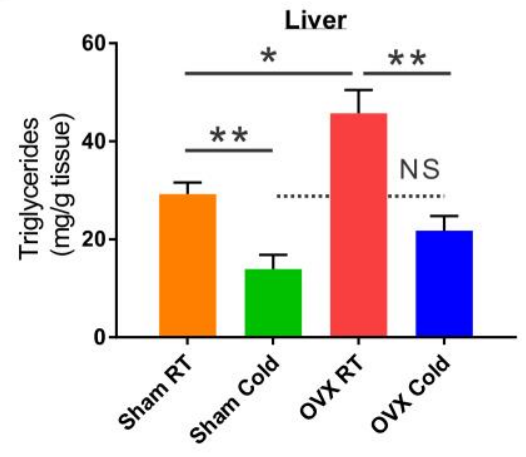




\section{Fig. 5}

a

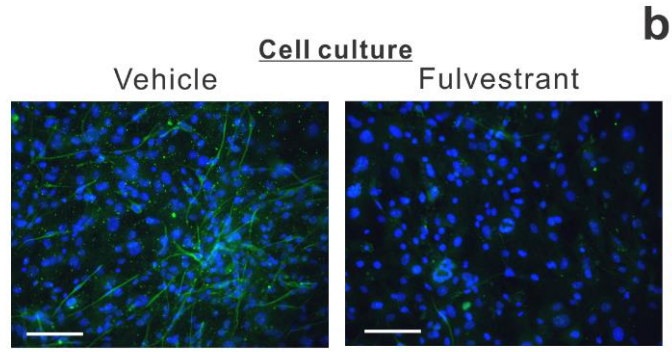

C

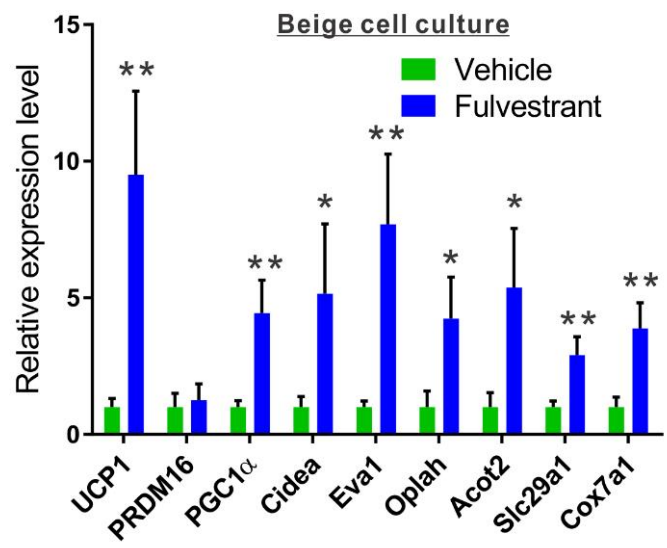

g

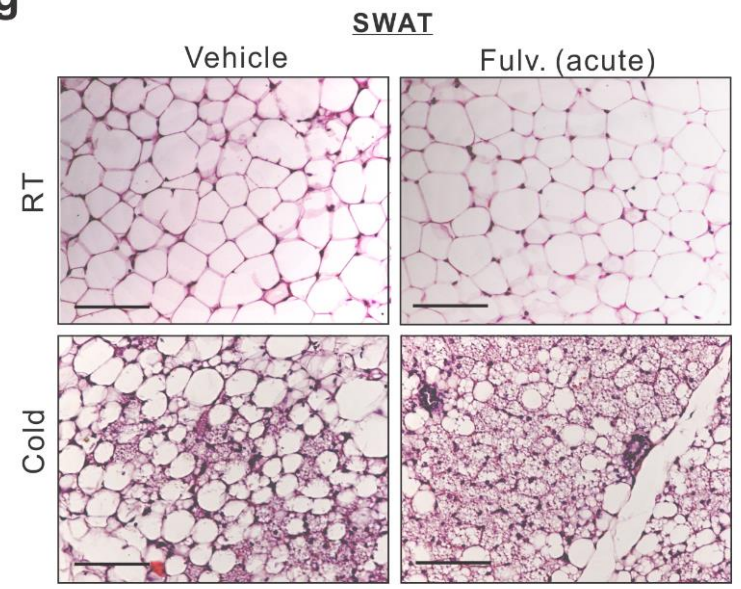

b

d
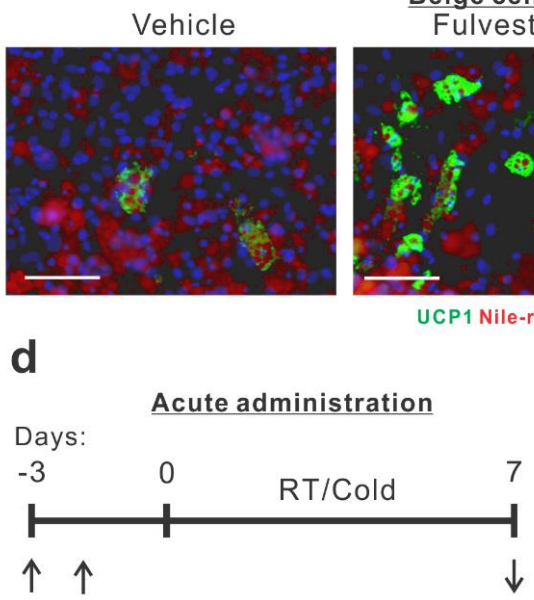

Vehicle / Fulvestrant Analysis

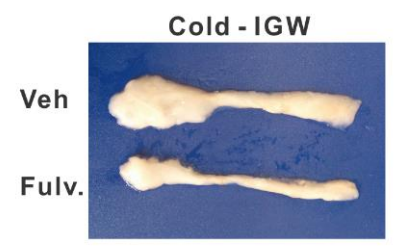

h

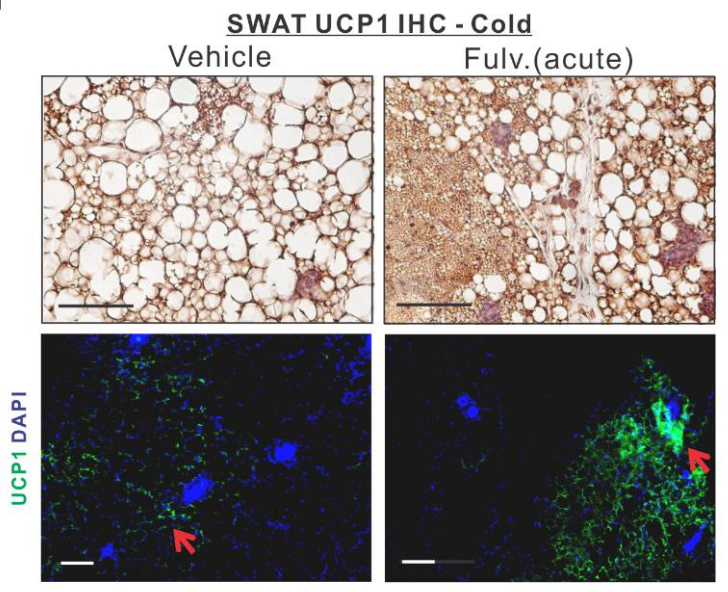

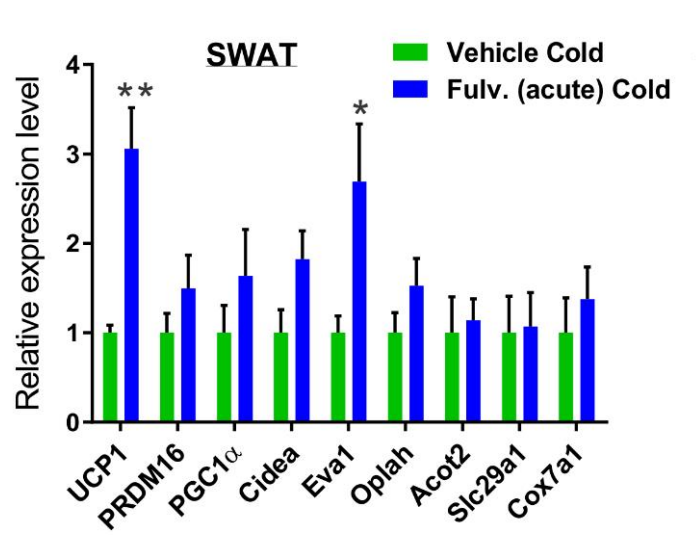

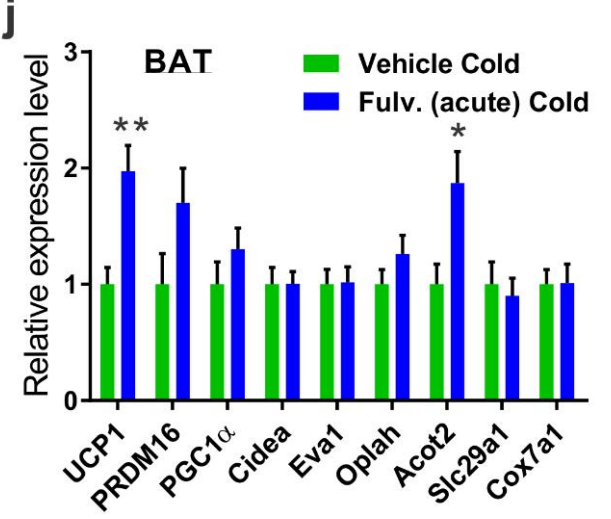

K

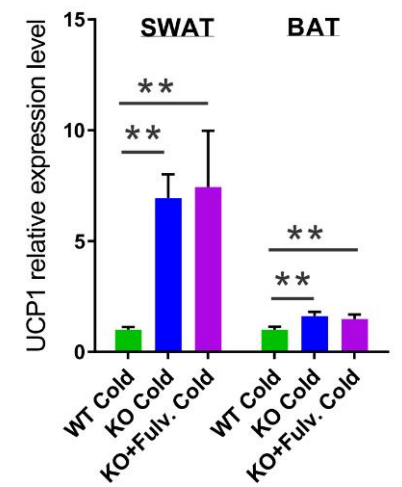




\section{Fig. S5}

a

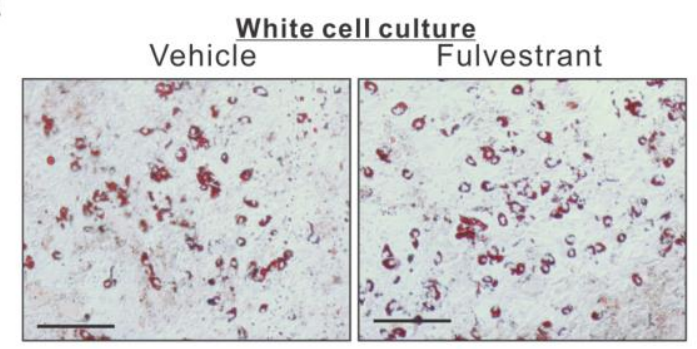

Oil Red 0

C

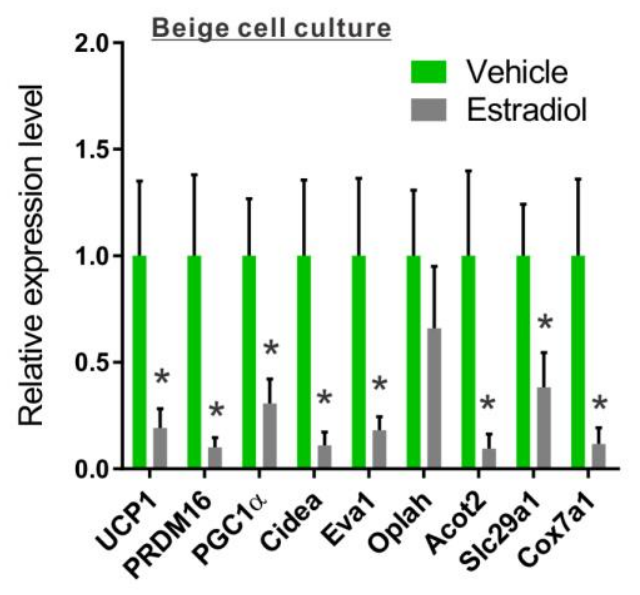

e

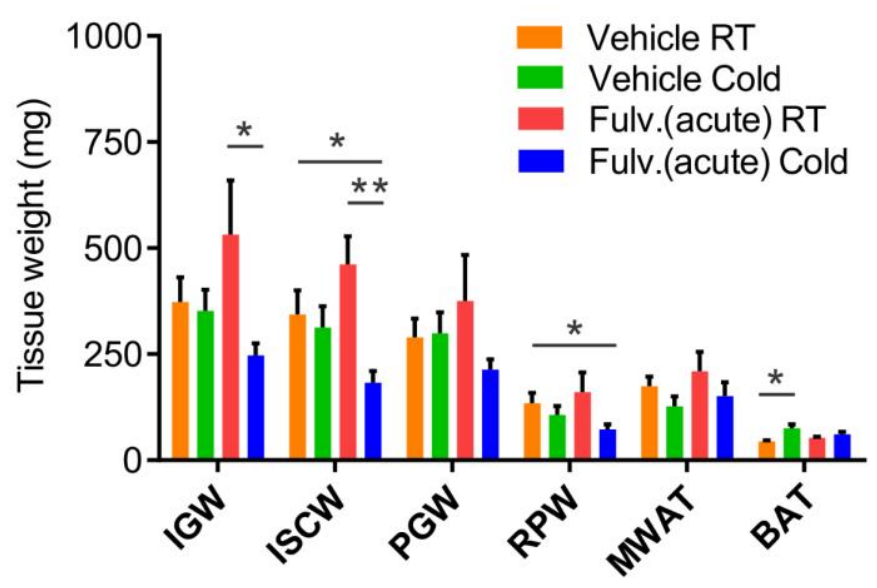

b White cell culture

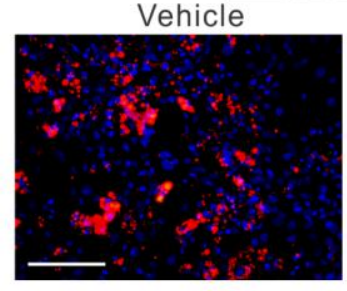

d

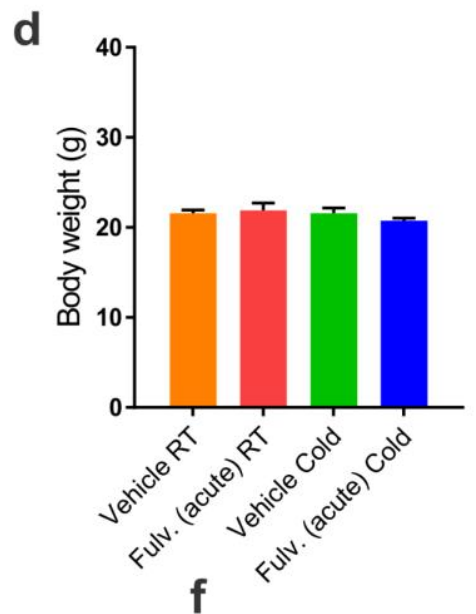

positive control
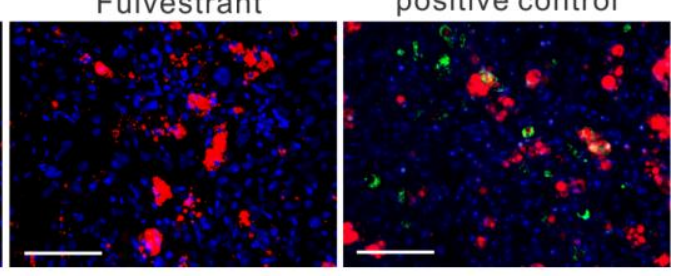

UCP1 Nile-red DAPI

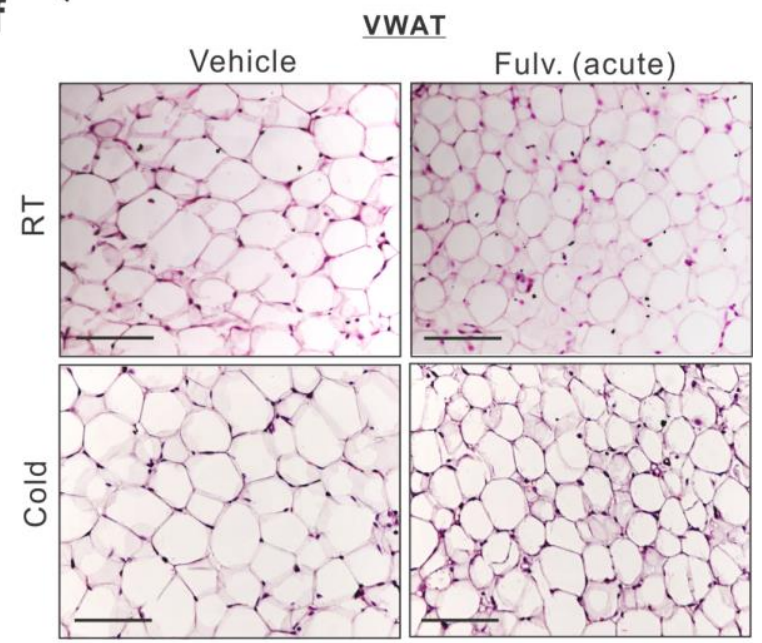


Fig. 6

a

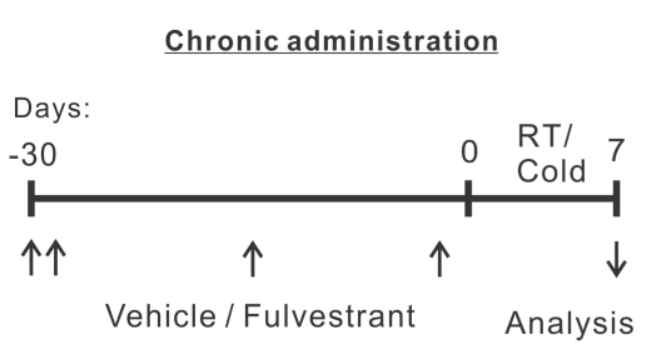

d

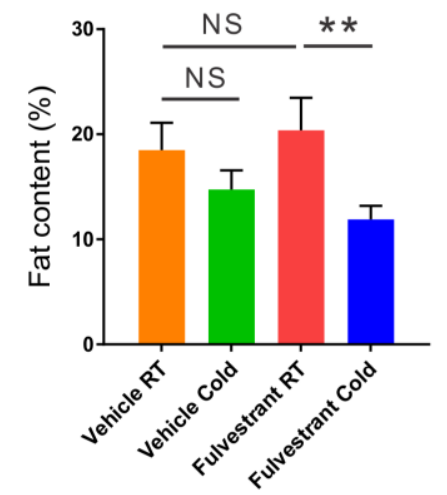

9

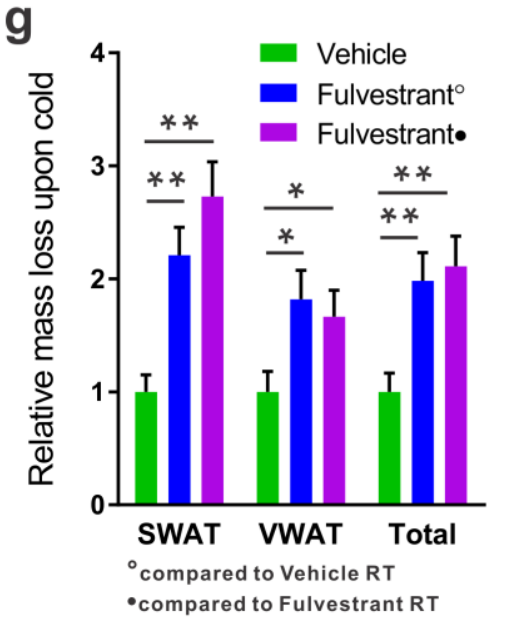

b

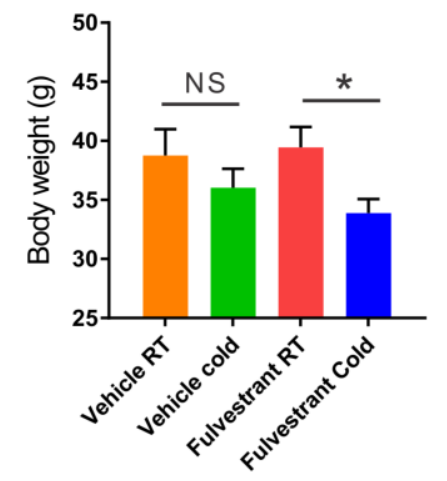

C

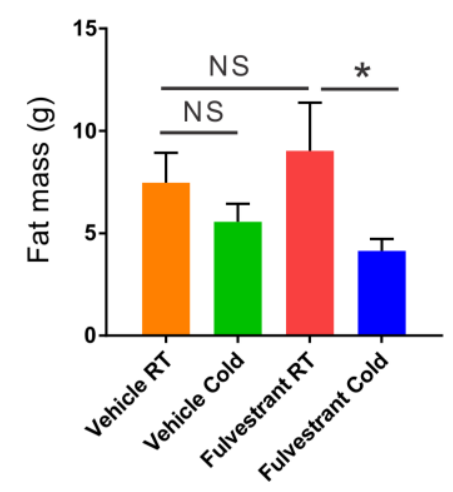

e

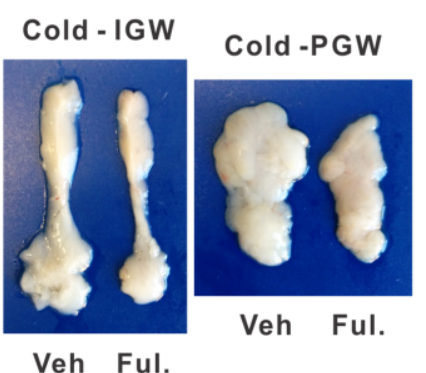

h

f
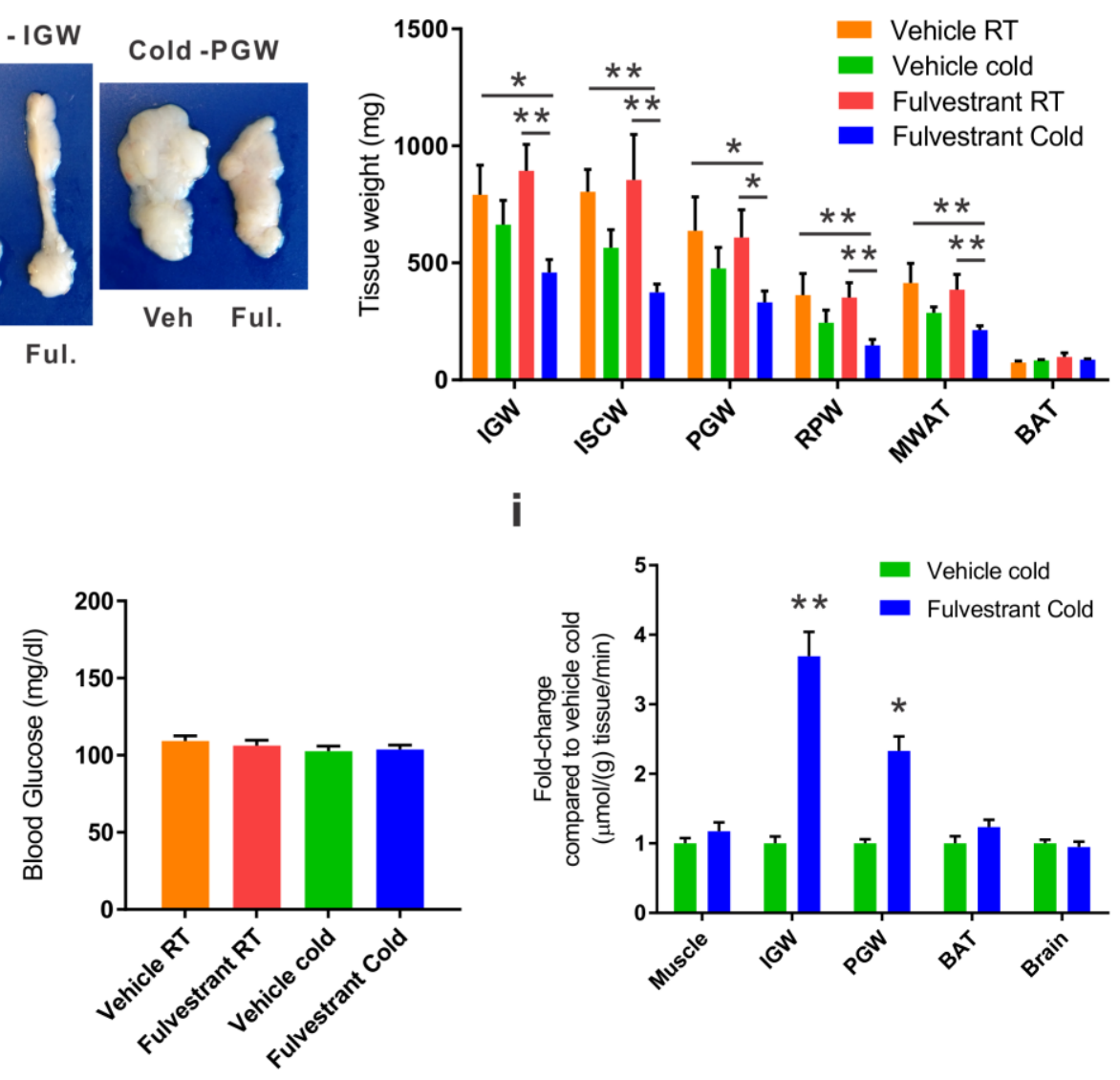


\section{Fig. S6}

a
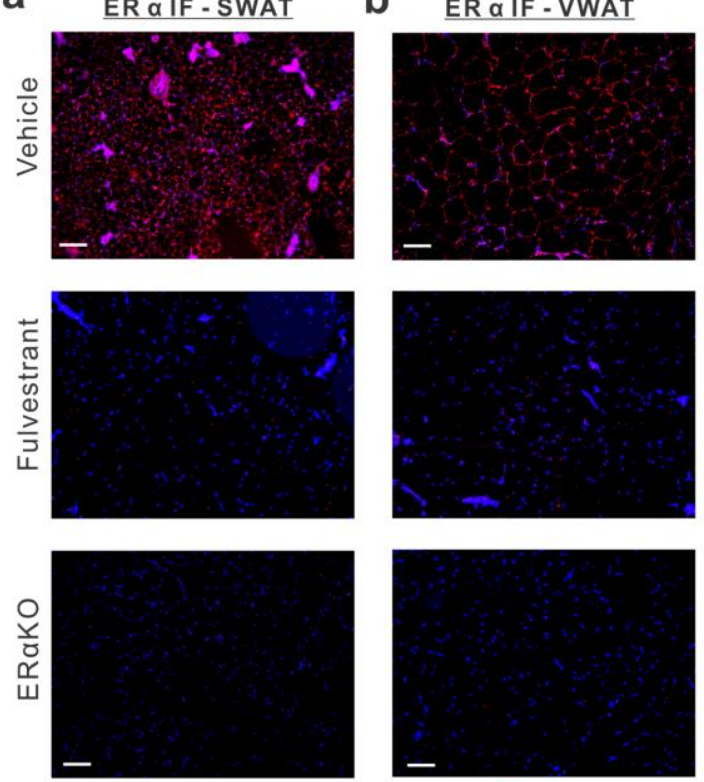

ER DAPI

f
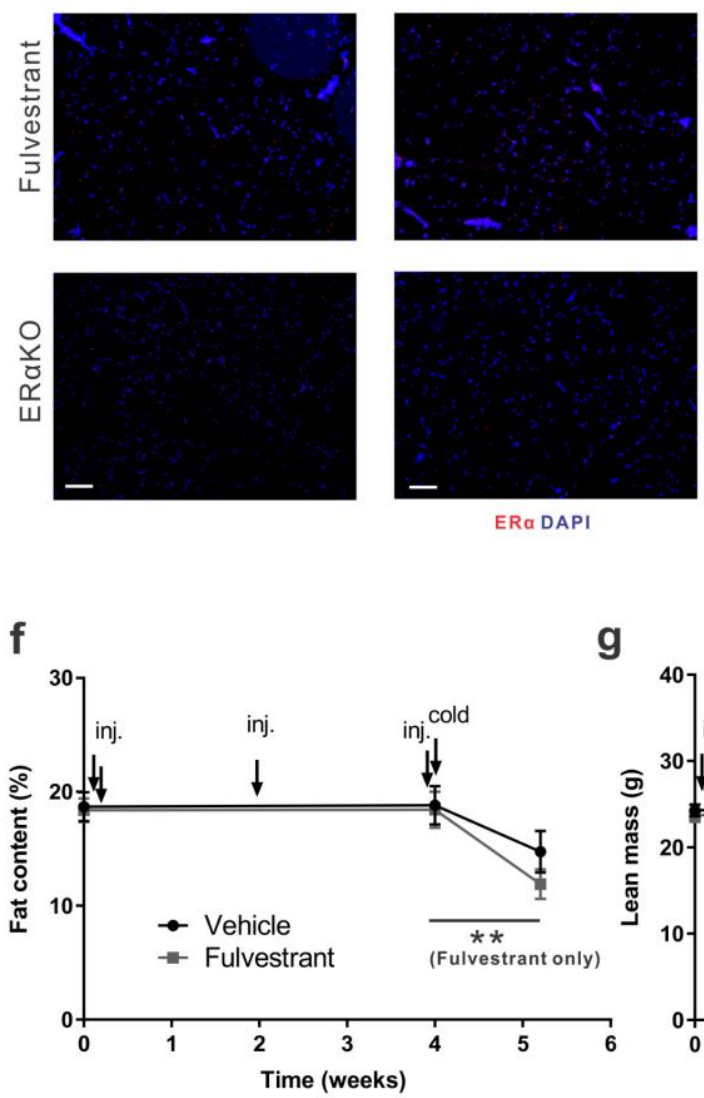

g
C
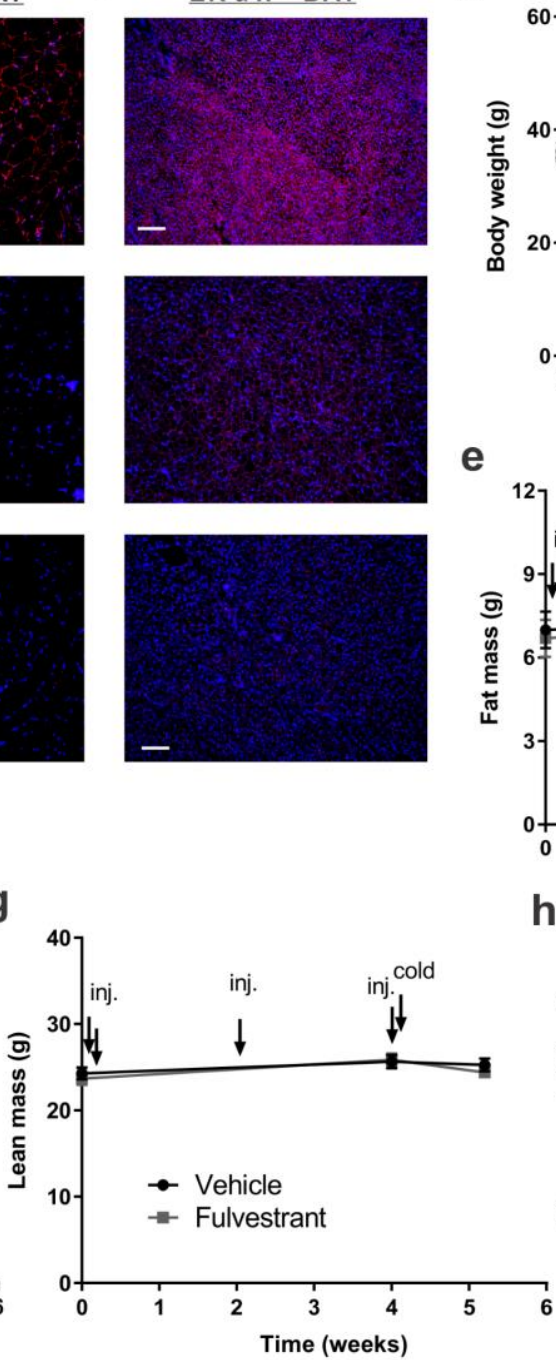

d

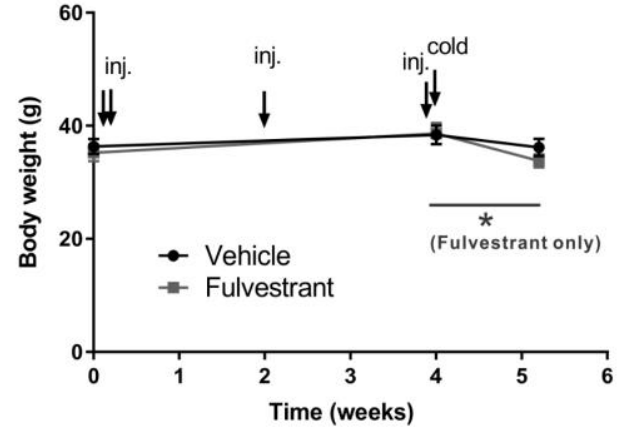

e

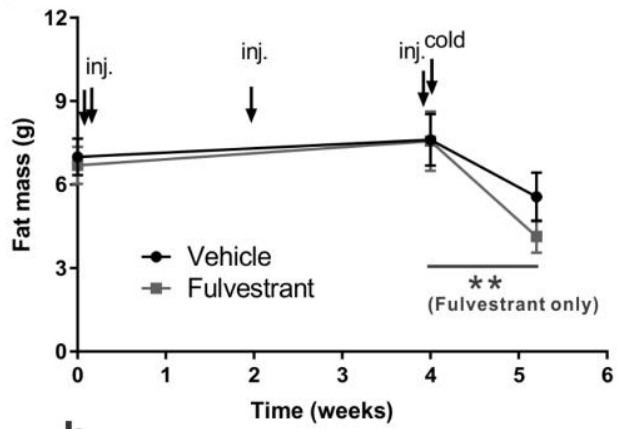

h

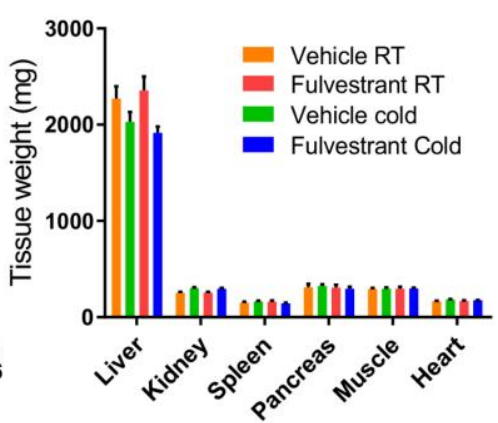


not certified by peer review) is the author/funder, who has granted bioRxiv a license to display the preprint in perpetuity. It is made availa Fig. under aCC-BY-NC-ND 4.0 International license.

a

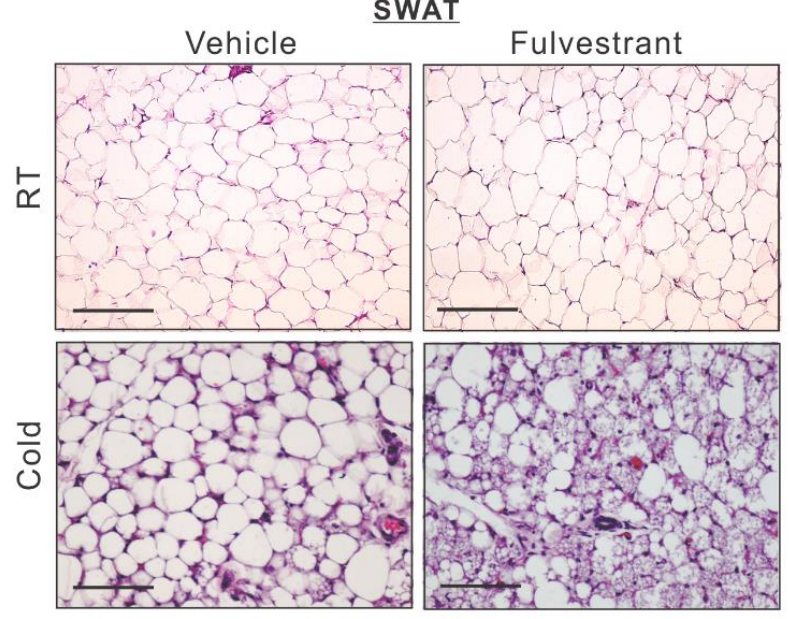

C

SWAT UCP1 - Cold
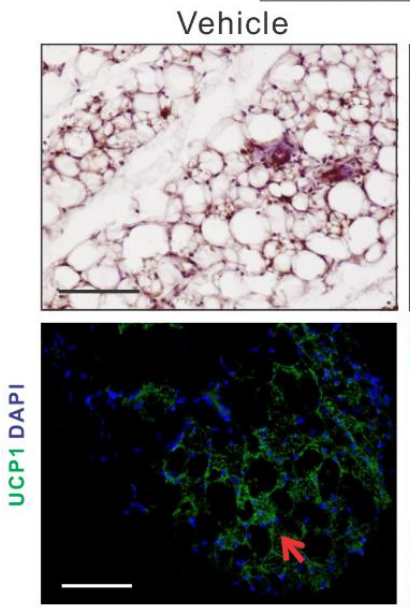

e

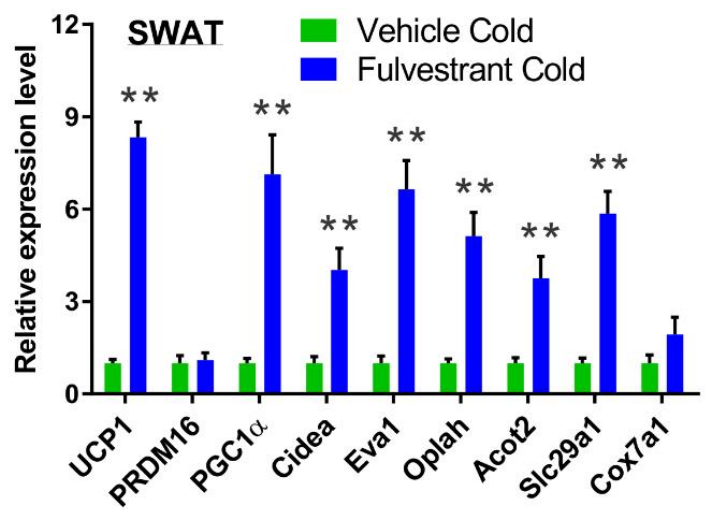

g
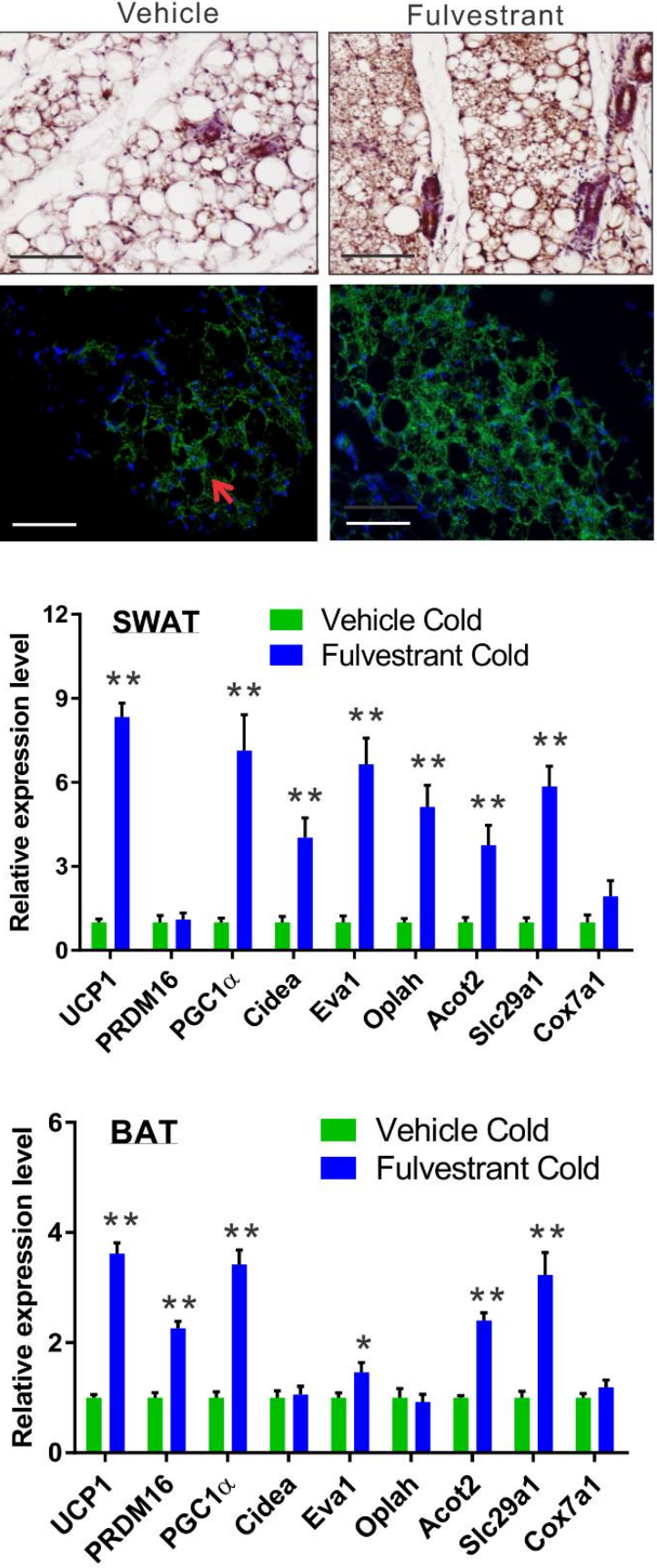

b

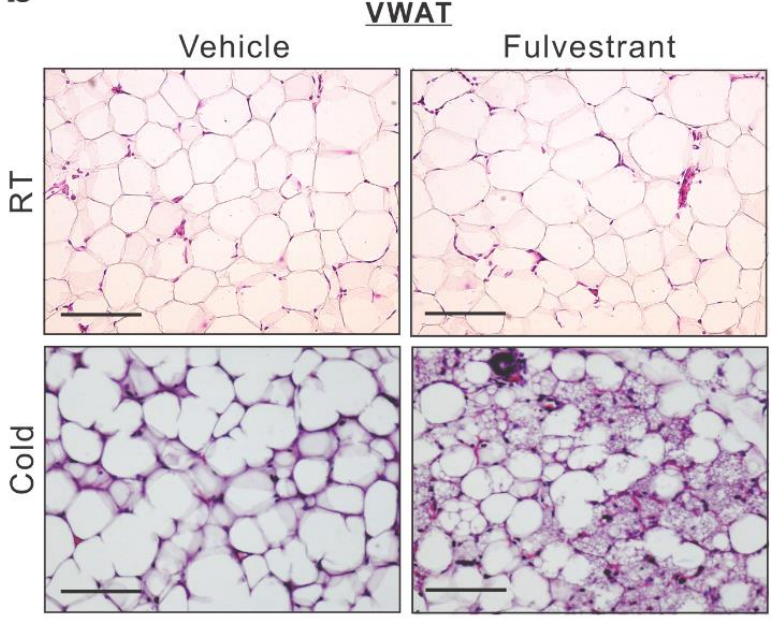

d
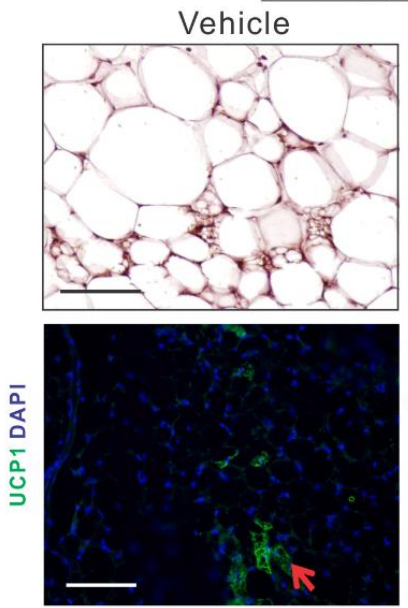

f

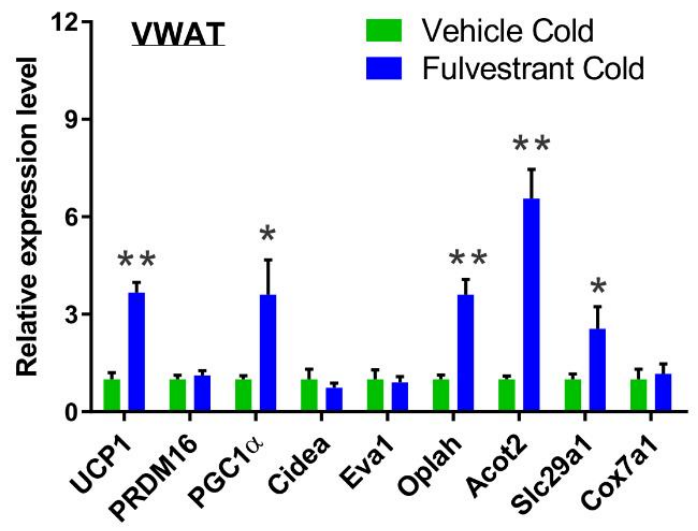

h

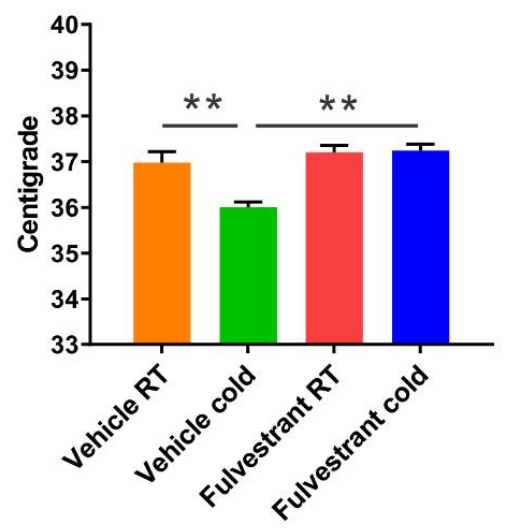




\section{Fig. S7}

a

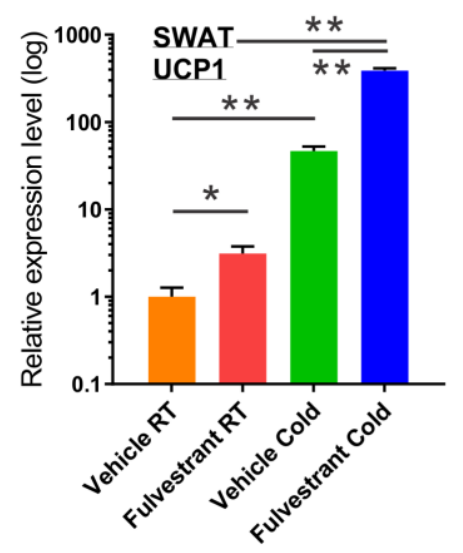

b

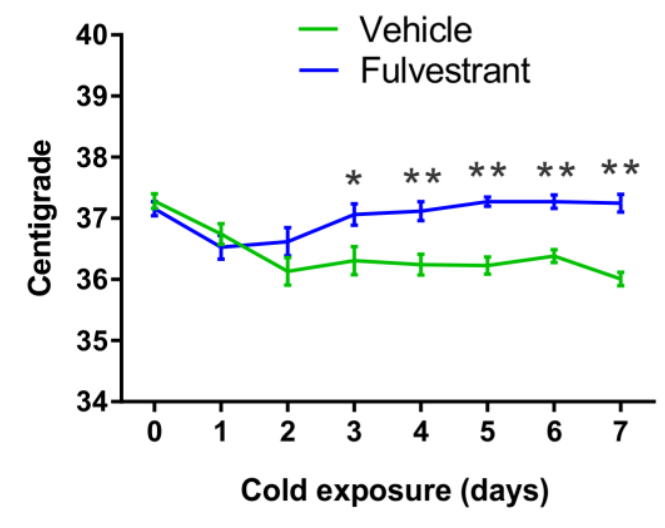




\section{Fig. 8}
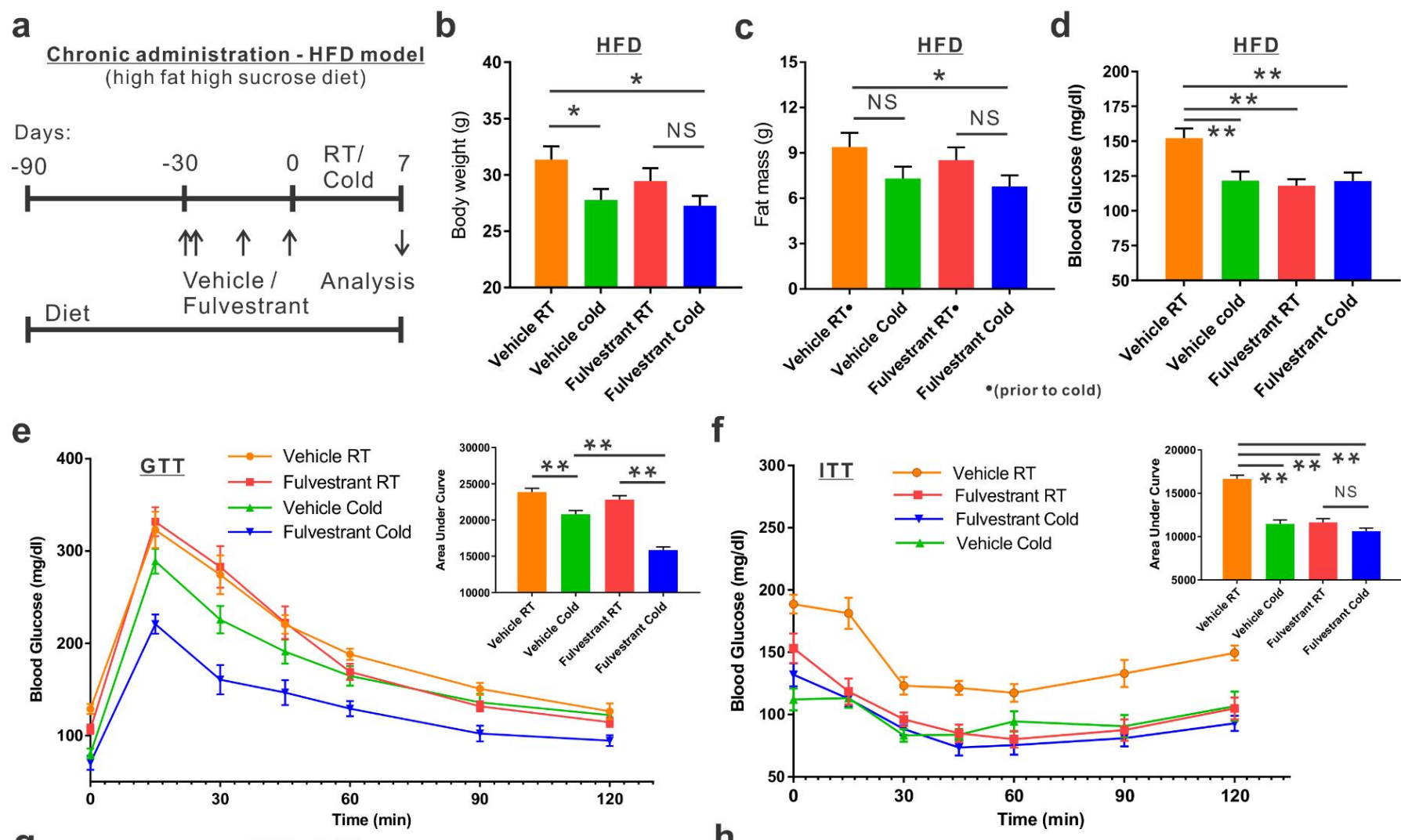

f

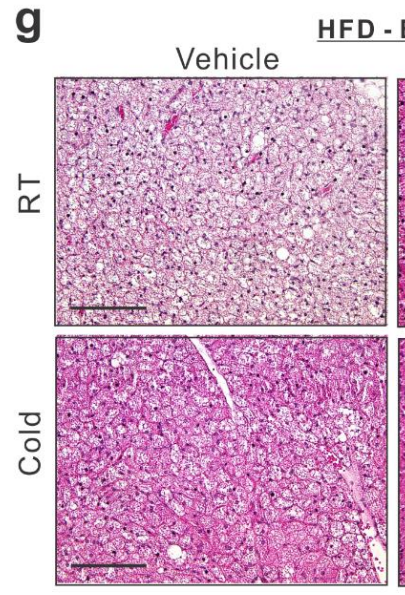

Fulvestrant

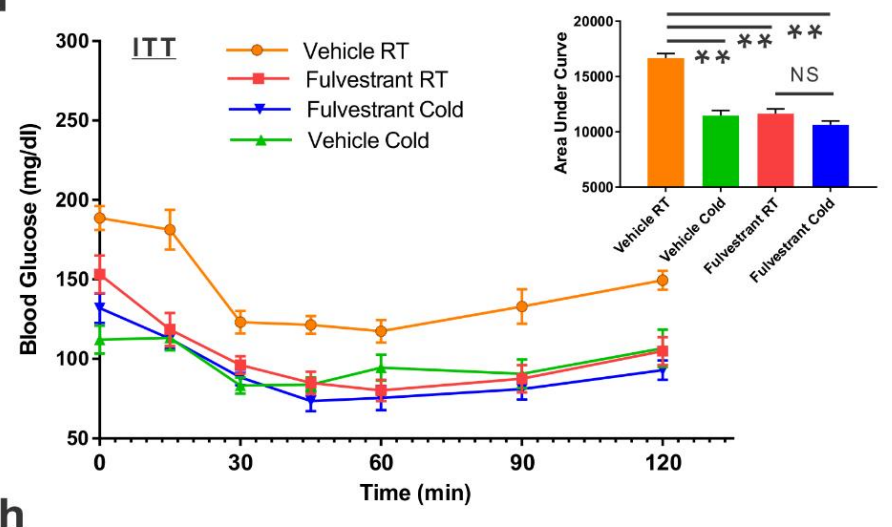

i
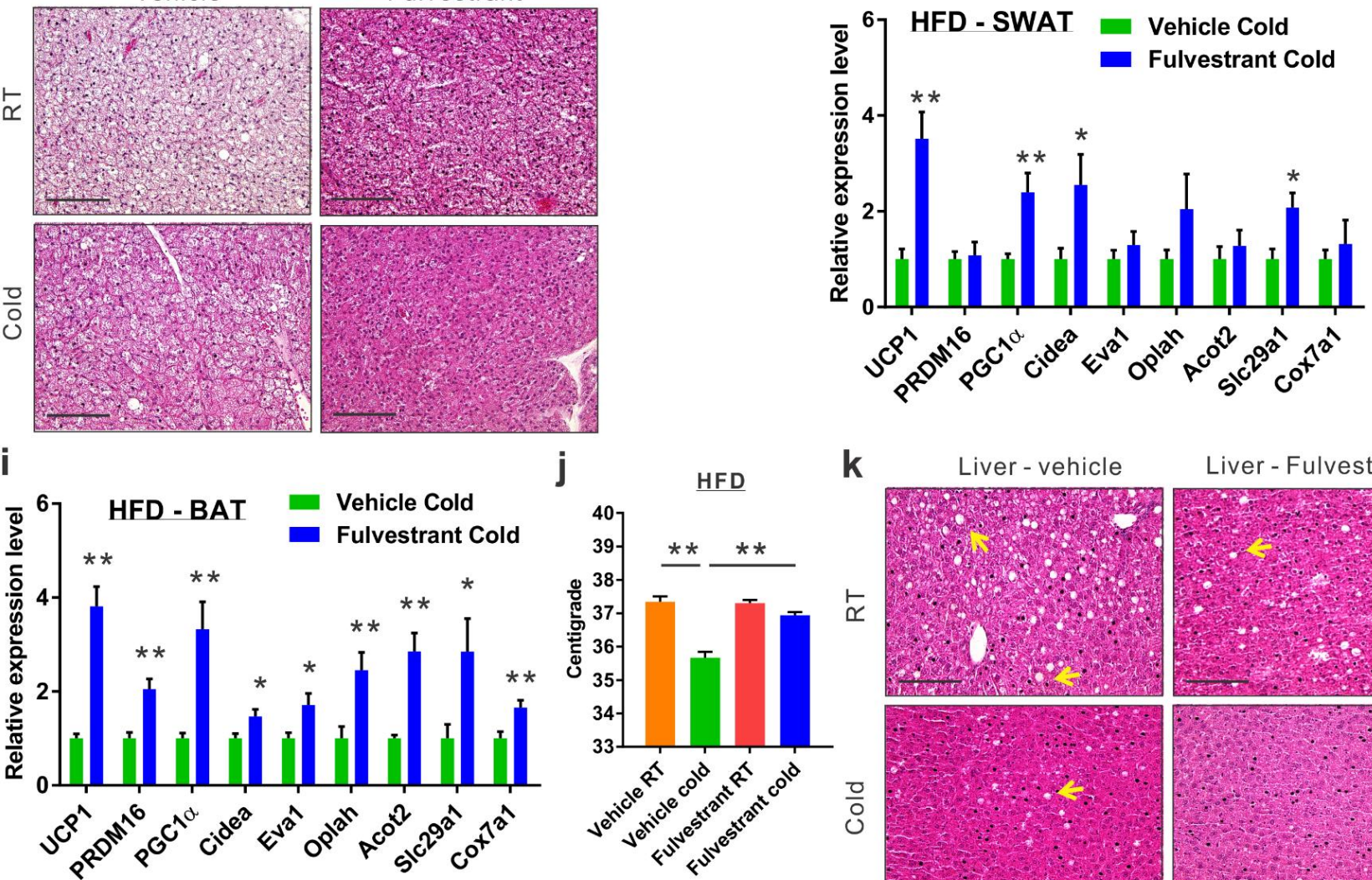

k

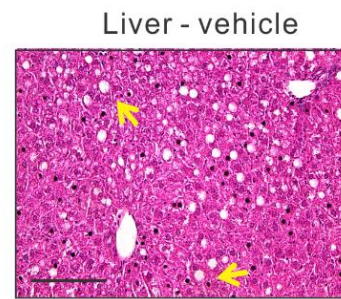

Liver - Fulvestrant

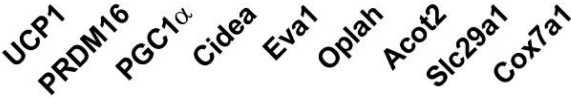
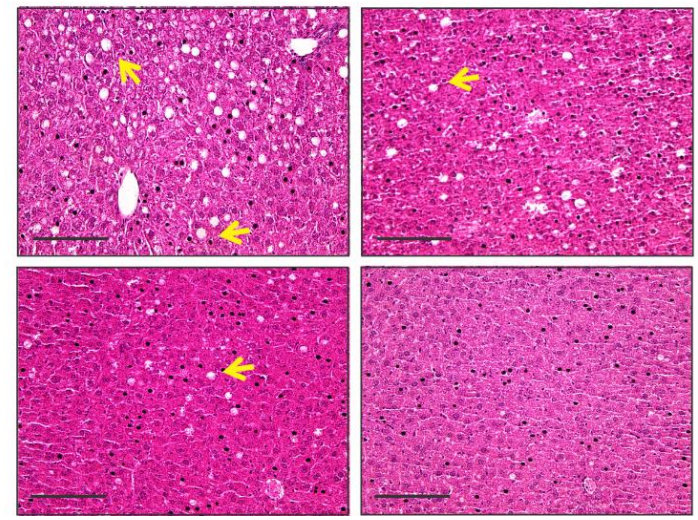


\section{Fig. S8}

a

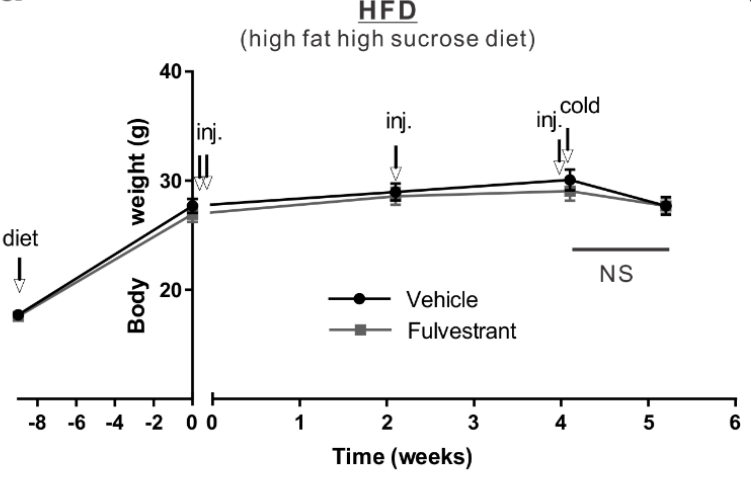

b
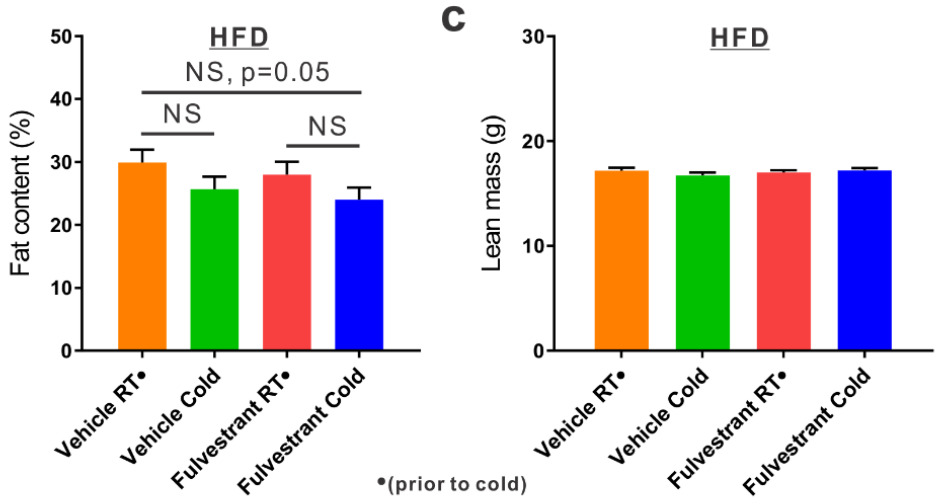

d

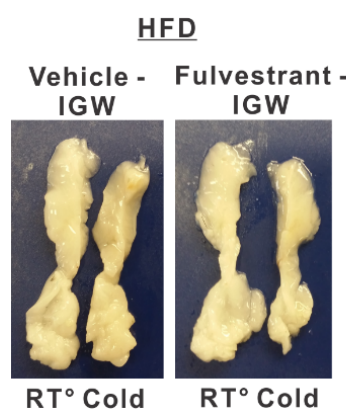

${ }^{\circ}$ (in parallel to cold)

g

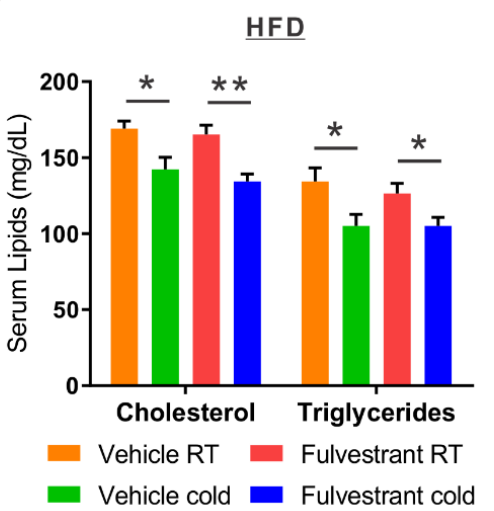

j

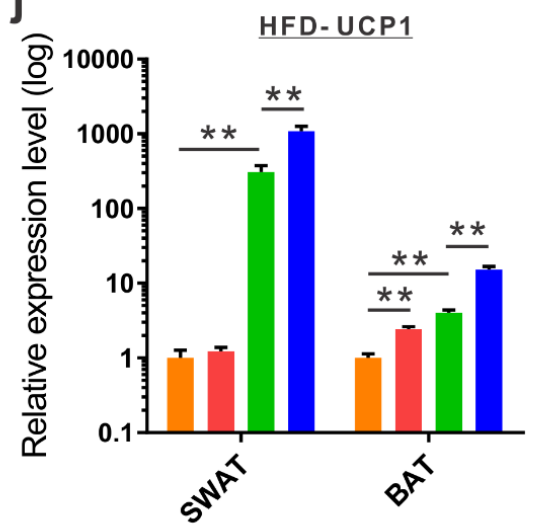

Vehicle RT Vehicle Cold

Fulvestrant RT $\boldsymbol{D}$ Fulvestrant Cold
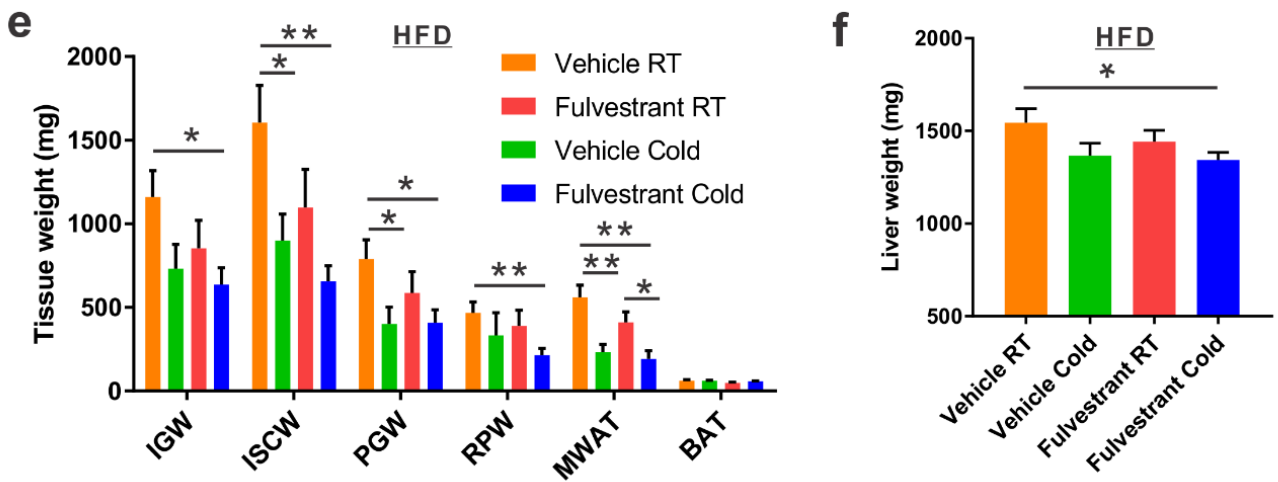

h

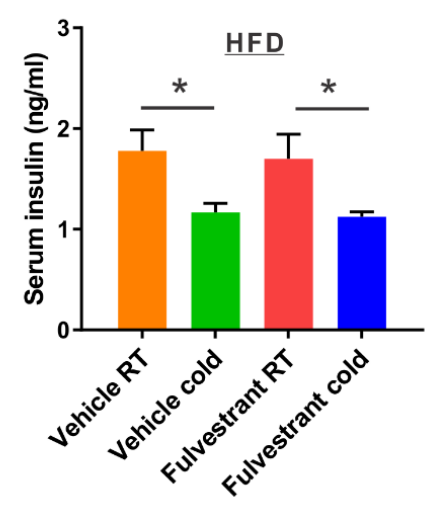

i

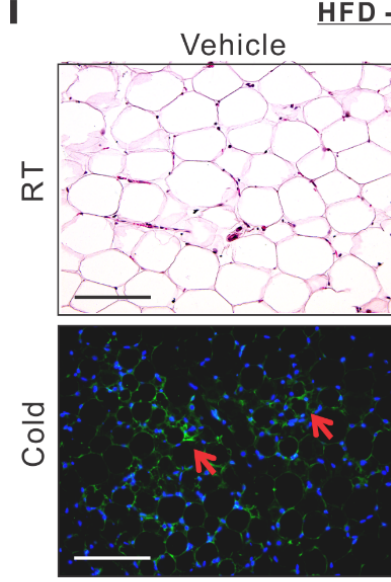

HFD - SWAT

k

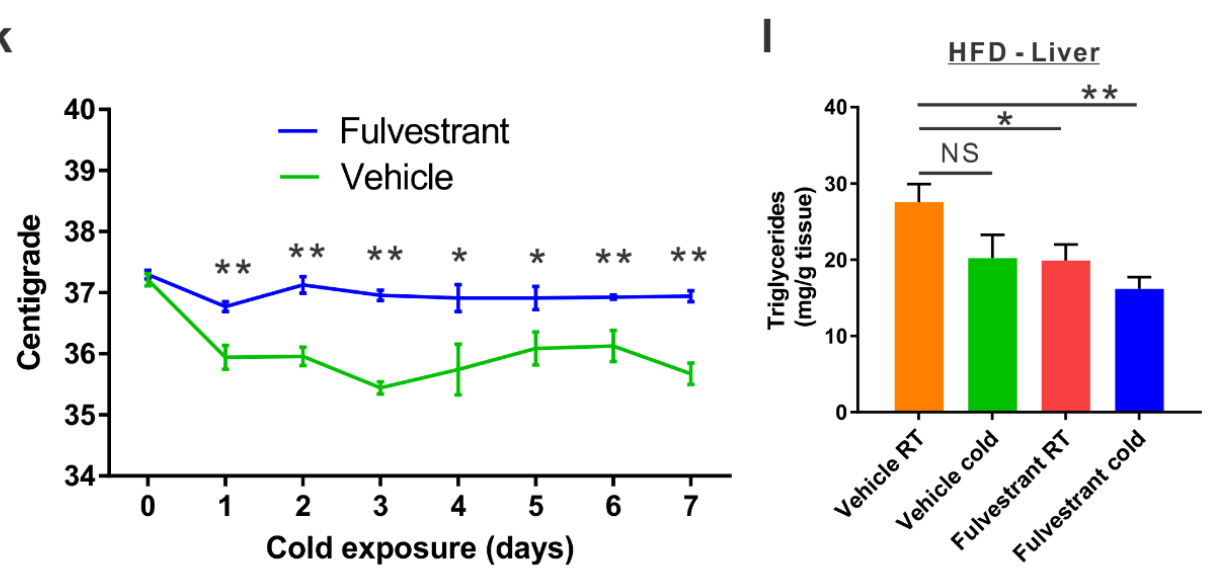


Fig. 9

a

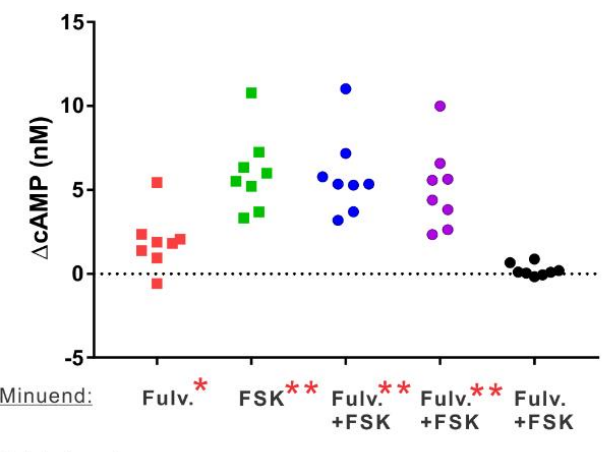

Subtrahend: Con. Con. Con. Fulv. FSK b

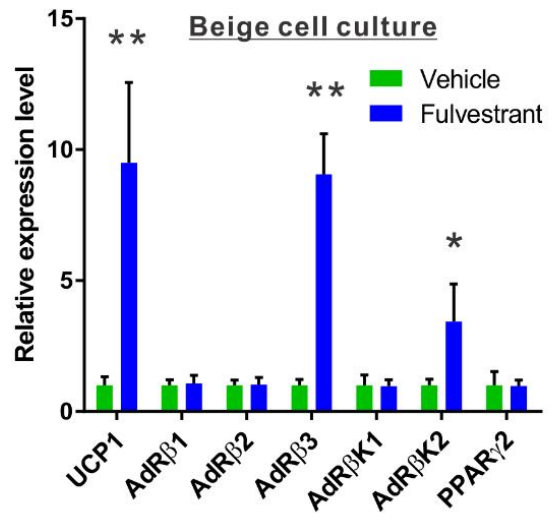

C

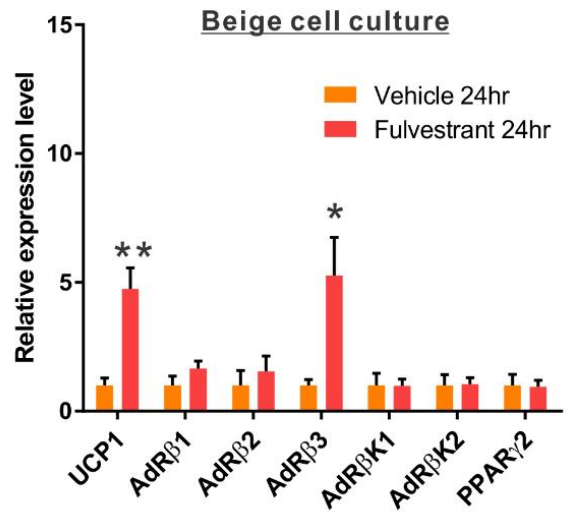

d

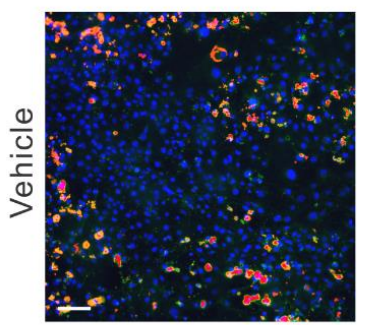

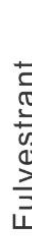

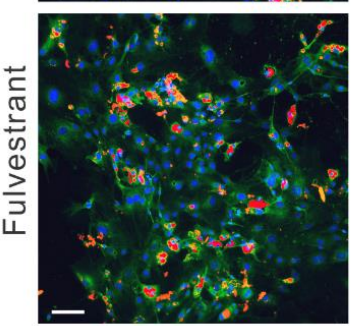

e
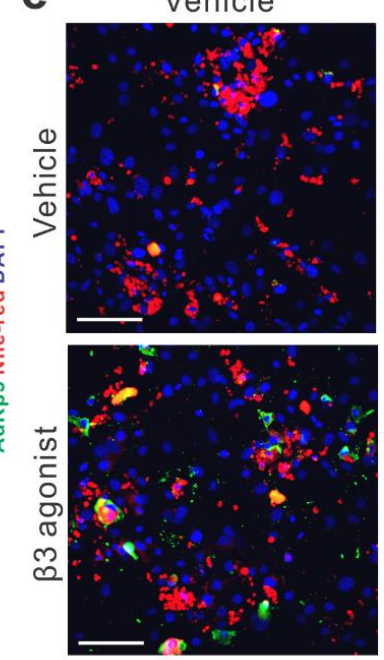

Fulvestrant
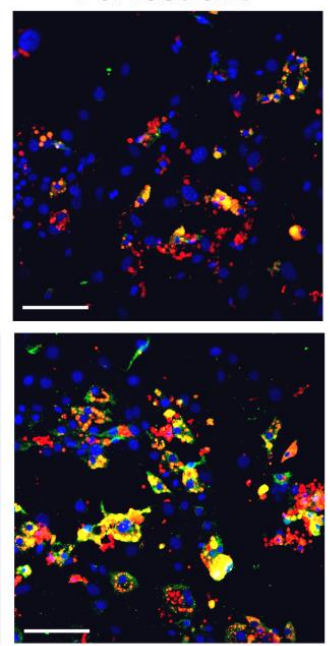

f Norepinephrine (NE)
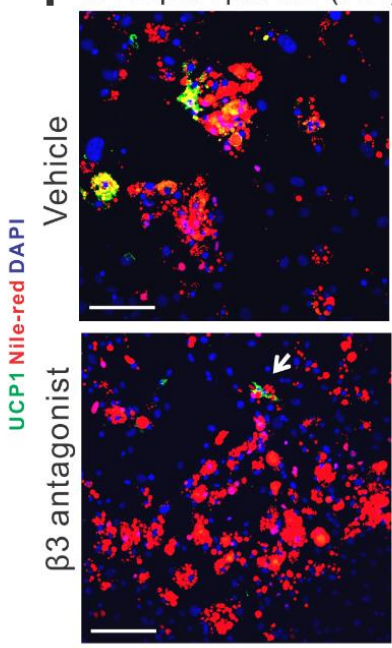
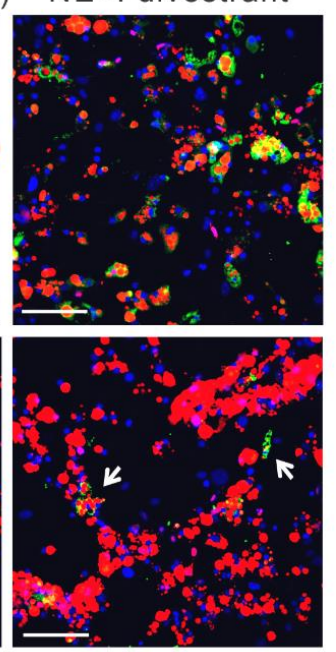

g Days: -3

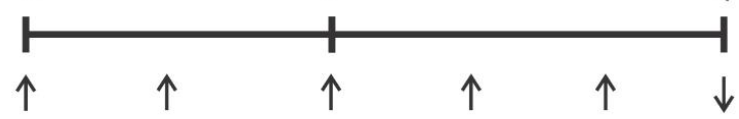

Vehicle / Fulvestrant Vehicle / $\beta 3$ agonist Analysis

h

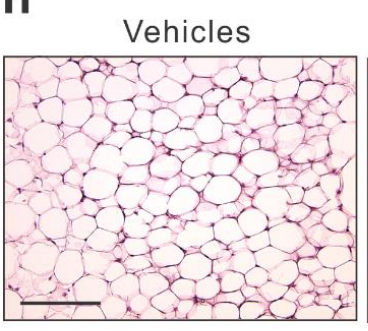

$\mathrm{KO}+$ vehicle

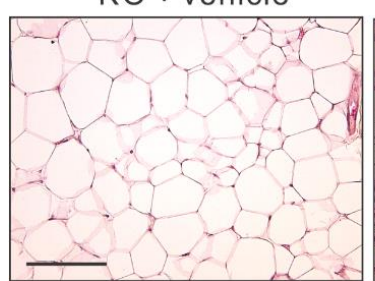

SWAT

$\beta 3$ agonist

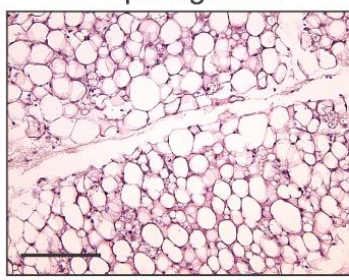

SWAT

$\beta 3$ agonist

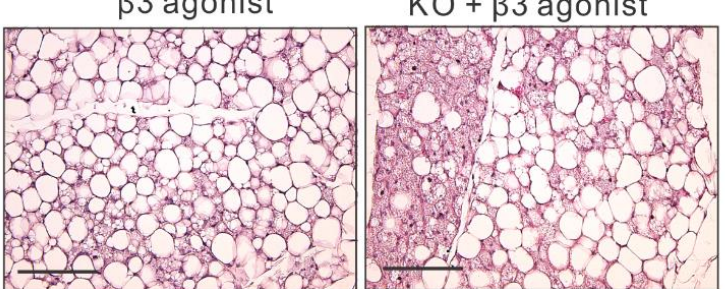

Fulv. $+\beta 3$ agonist

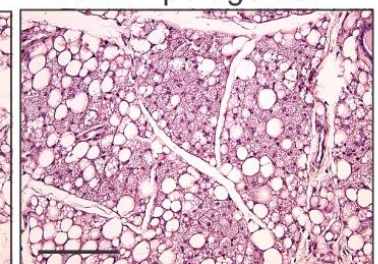

k

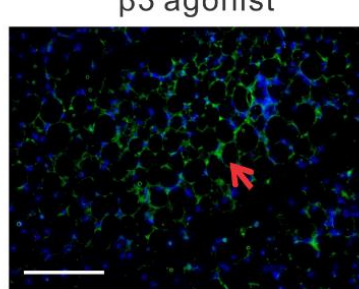

$\beta 3$ agonist

\section{SWAT UCP1}

$\beta 3$ agonist

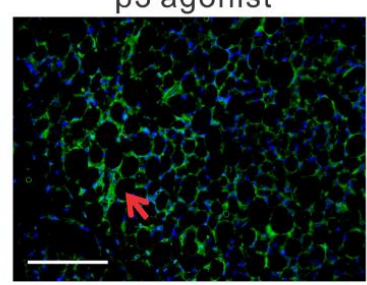

Fulv. $+\beta 3$ agonist

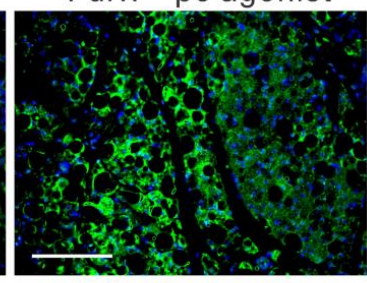

UCP1 DAPI

SWAT UCP1

$\mathrm{KO}+\beta 3$ agonist

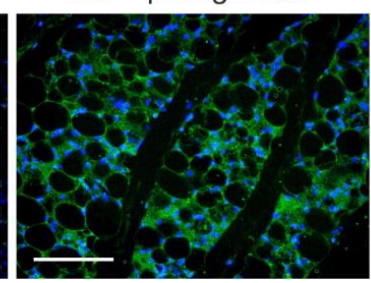




\section{Fig. S9}
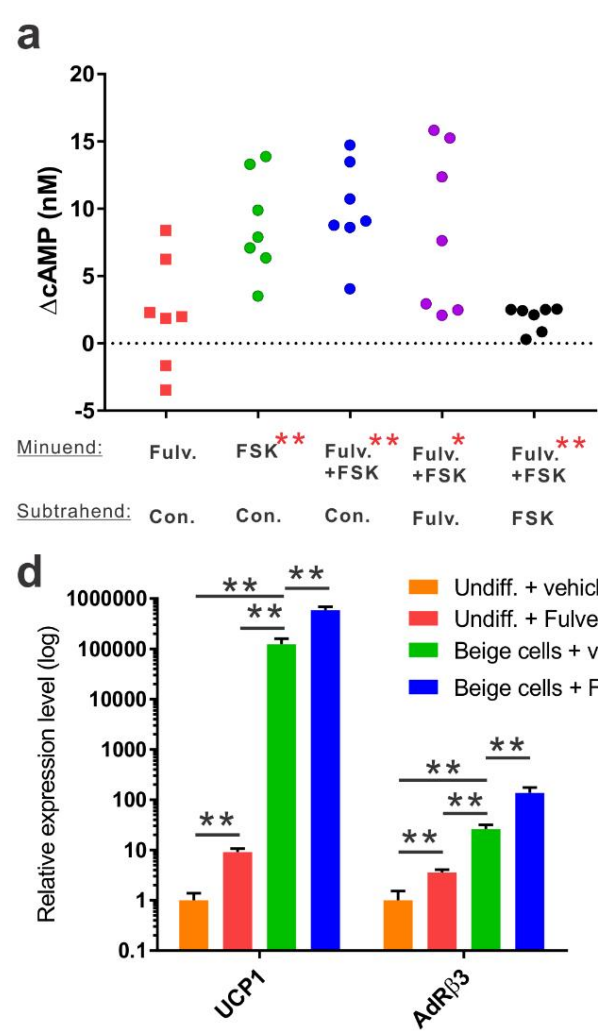

g
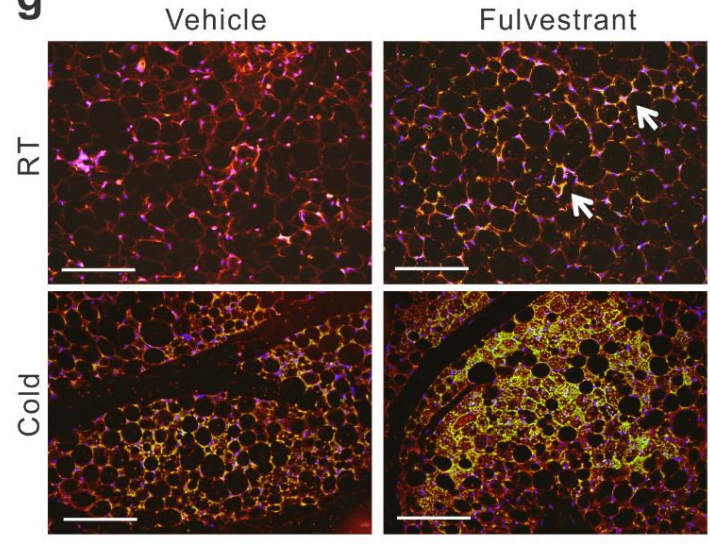

j

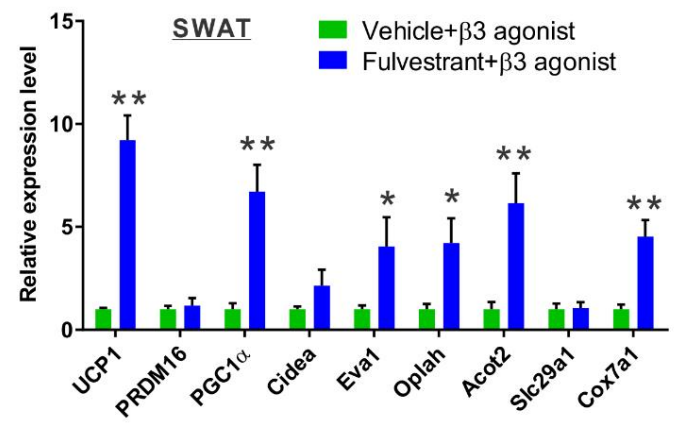

b

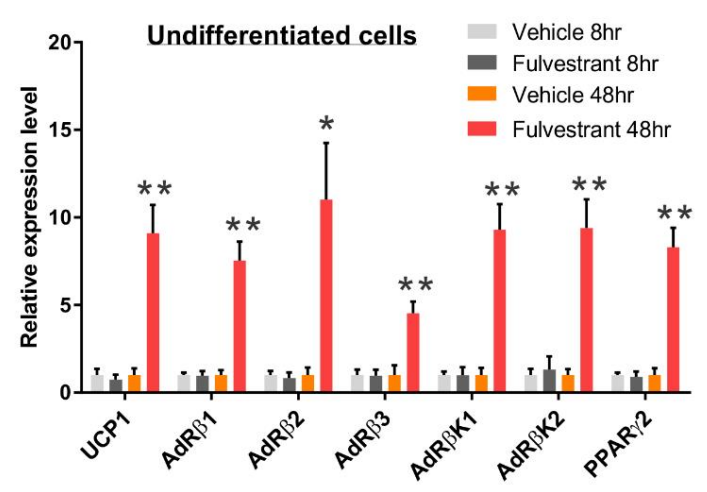

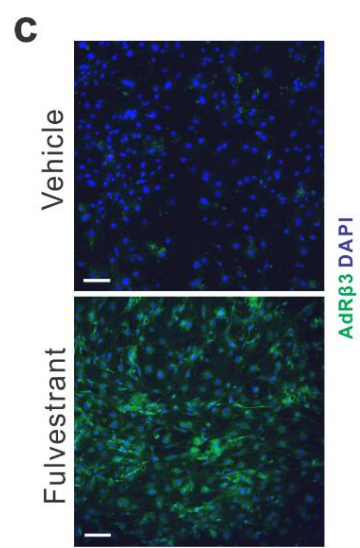
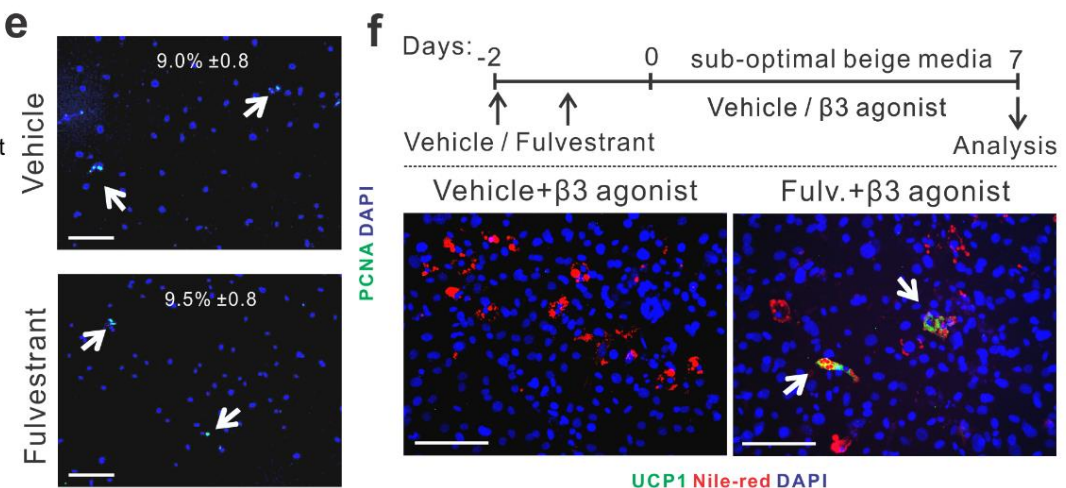

UCP1 Nile-red DAPI

h
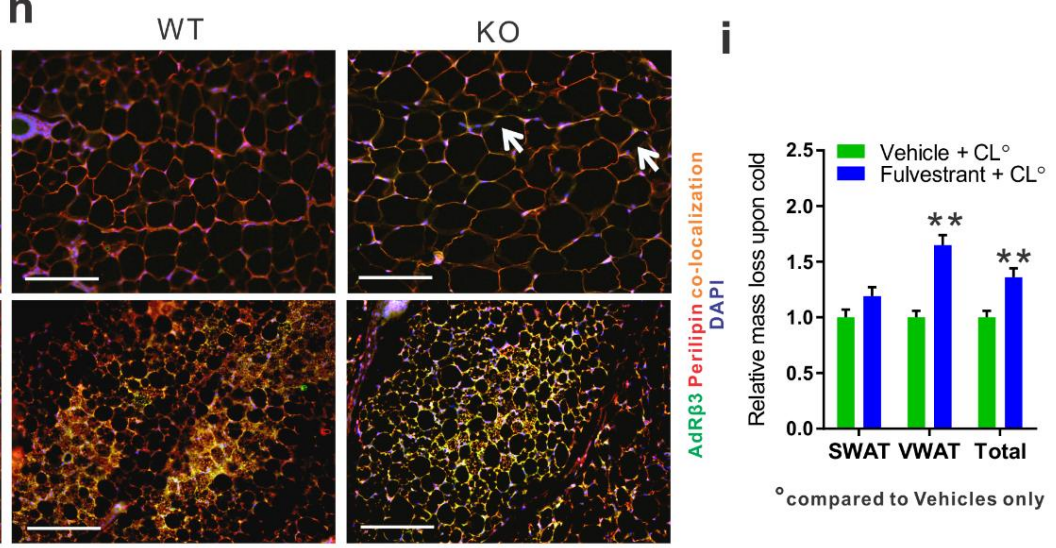

k

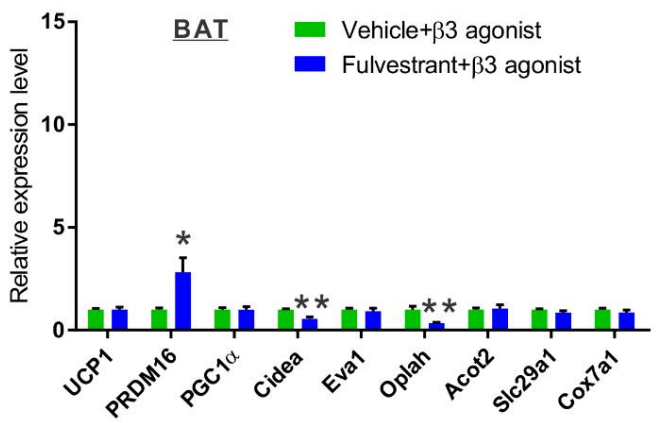




\section{Fig. 10}

a)
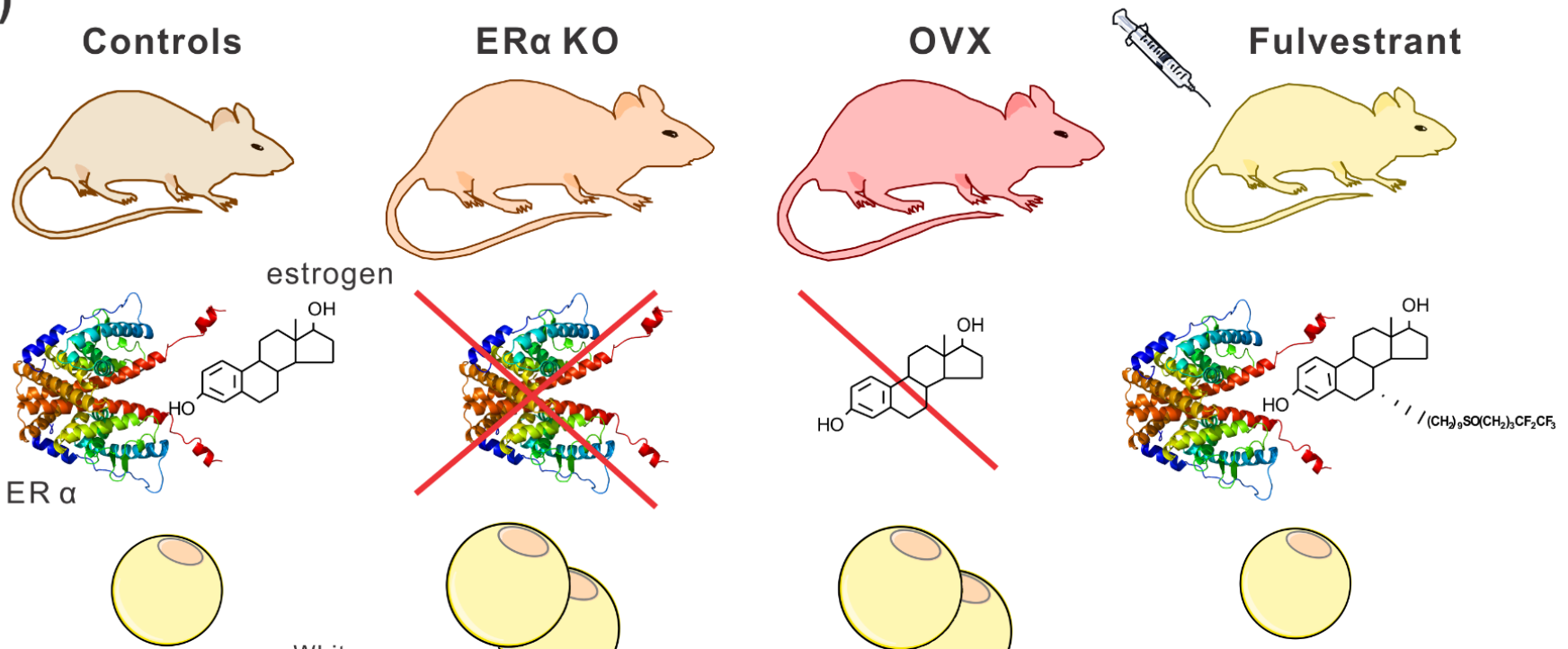

White Adipocytes
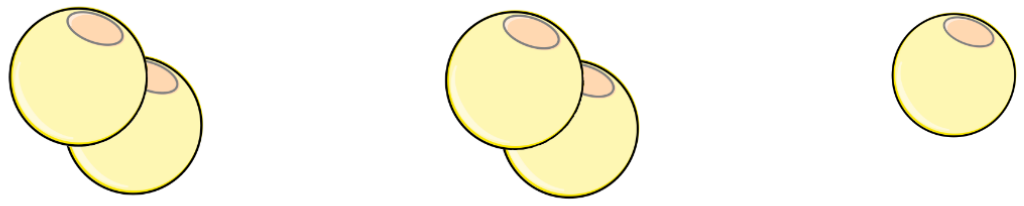

b)
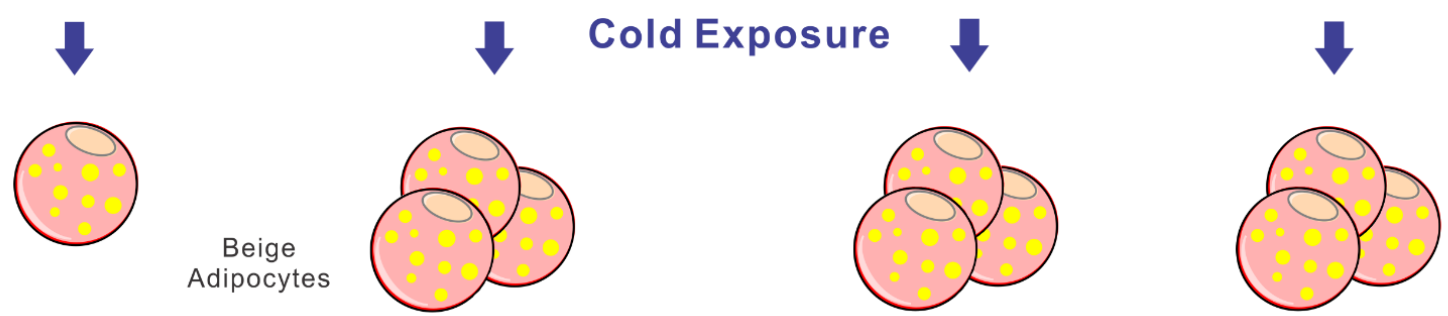

\begin{tabular}{|c|c|c|c|c|c|c|c|c|}
\hline $\begin{array}{l}\text { Cold- } \\
\text { exposure }\end{array}$ & Adiposity & $\begin{array}{l}\text { Hyper- } \\
\text { glycemia }\end{array}$ & \begin{tabular}{|l|} 
Glucose \\
Sensitivity
\end{tabular} & \begin{tabular}{|l|} 
Insulin \\
Sensitivity
\end{tabular} & \begin{tabular}{|l} 
Hyperchol- \\
estrolemia
\end{tabular} & \begin{tabular}{|l|} 
Hypertrigl- \\
yceridemia
\end{tabular} & \begin{tabular}{|l|}
$\begin{array}{l}\text { Hepto- } \\
\text { steatosis }\end{array}$ \\
\end{tabular} & \multirow{3}{*}{$\begin{array}{l}\text { N/A- } \\
\text { does not charaterize } \\
\text { the model }\end{array}$} \\
\hline ER $\alpha$ KO & $\downarrow$ & $\downarrow$ & $\uparrow$ & $\mathbf{t}$ & $\downarrow$ & $\downarrow$ & $\mathrm{N} / \mathrm{A}$ & \\
\hline ovx & $\downarrow$ & $\downarrow$ & $\mathbf{t}$ & $\leftrightarrow$ & $\downarrow$ & $\downarrow$ & $\downarrow$ & \\
\hline Fulvestrant & $\downarrow$ & $\mathrm{N} / \mathrm{A}$ & ND & ND & $\mathrm{N} / \mathrm{A}$ & $\mathrm{N} / \mathrm{A}$ & $\mathrm{N} / \mathrm{A}$ & \multirow[t]{2}{*}{ ND-not determined } \\
\hline $\begin{array}{l}\text { Fulvestrant } \\
\text { (HFD) }\end{array}$ & $\downarrow$ & $\downarrow^{*}$ & $\uparrow$ & $\mathbf{t}^{*}$ & $\longleftrightarrow$ & $\leftrightarrow$ & $\downarrow$ & \\
\hline
\end{tabular}

c)

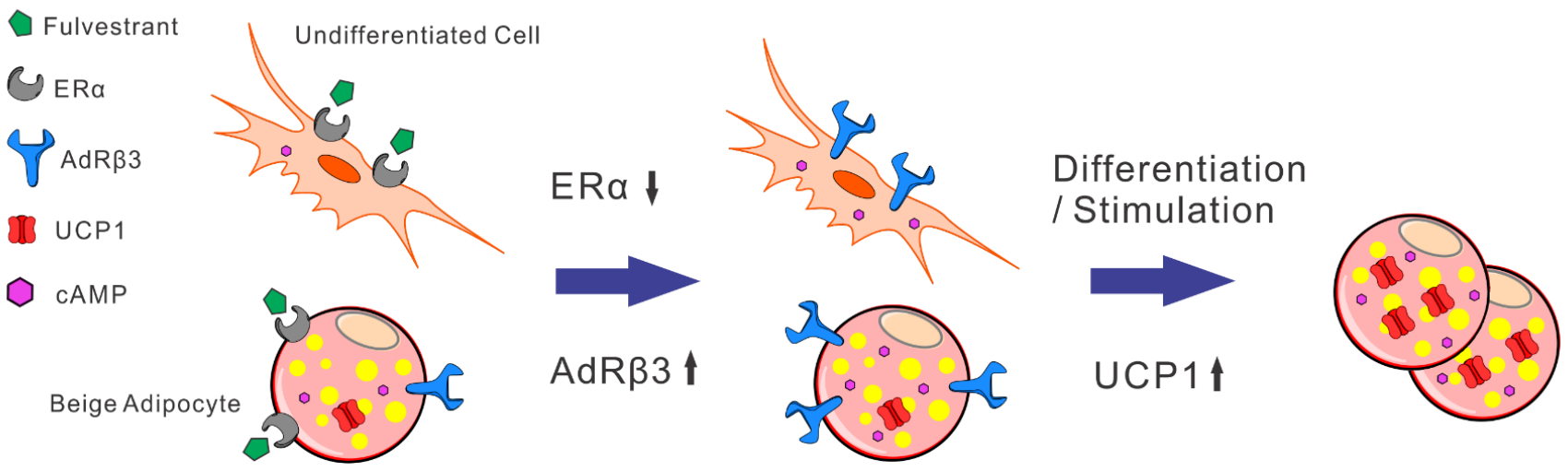

